Der Medizinischen Fakultät der Georg-August-Universität Göttingen eingereicht von Prof. Dr. med. U. Fischer

\title{
Differenzierung von Befunden der Kategorien BIRADS 3 und BIRADS 4 in der hochauflösenden Mamma-MRT
}

\author{
INAUGURAL - DISSERTATION \\ zur Erlangung des Doktorgrades für Zahnheilkunde \\ der Medizinischen Fakultät \\ der Georg-August-Universität zu Göttingen
}

vorgelegt von

Fabian Ruhland

aus Göttingen

Göttingen 2011 
Dekan:

Prof. Dr. med. C. Frömmel

I. Berichterstatter: Prof. Dr. med. U. Fischer

II. Berichterstatter/in: Prof. Dr. med. G. Emons

III. Berichterstatter/in: Prof. Dr. med. dent. R. Mausberg

Tag der mündlichen

Prüfung:
17.04.2012 


\section{Inhaltsverzeichnis}

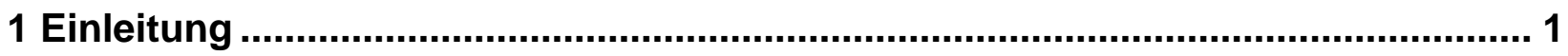

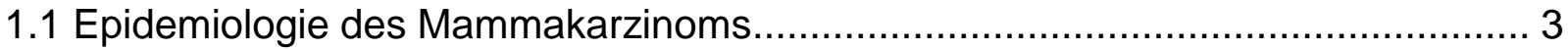

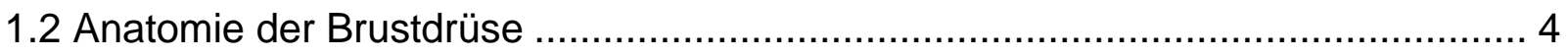

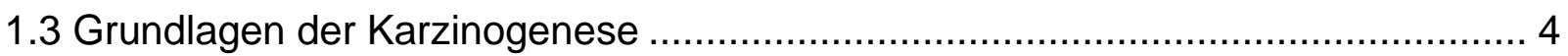

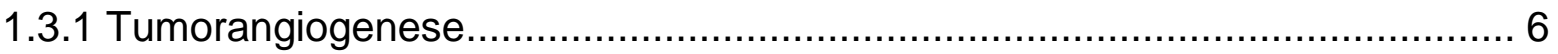

1.4 Maligne Erkrankungen der Brustdrüse ..................................................... 7

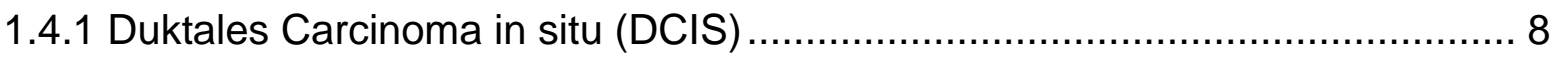

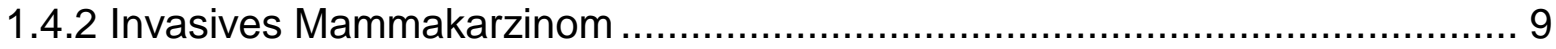

1.4.2.1 Invasiv duktales Mammakarzinom (IDC) ……................................... 9

1.4.2.2 Invasiv lobuläres Mammakarzinom (ILC) ………................................ 9

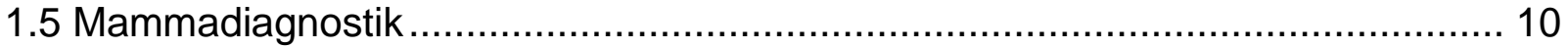

1.5.1 Klinische Untersuchung der Mamma ..................................................... 10

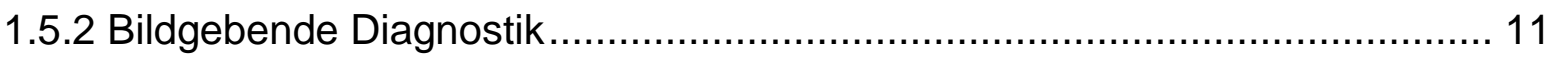

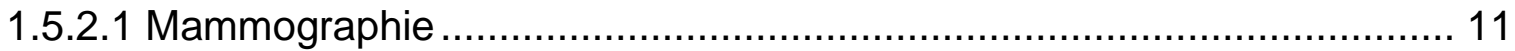

1.5.2.1.1 Befundbewertung - BI-RADS ${ }^{\mathrm{TM}}$-Klassifikation .............................. 13

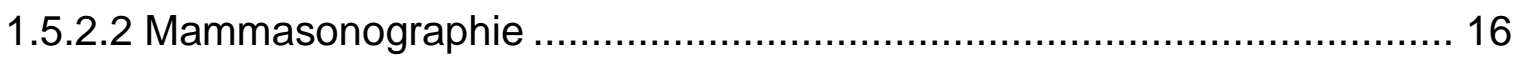

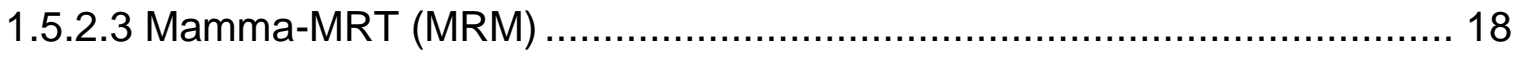

1.5.2.3.1 Terminologie und Auswertekriterien in der Mamma-MRT ............... 19

1.5.2.3.2 Kontrastmitteldynamik in der Mamma-MRT .................................. 20

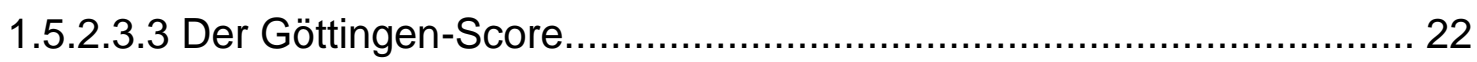

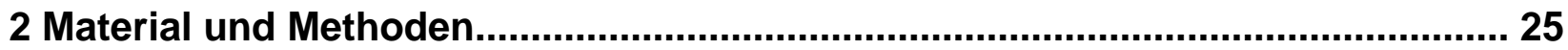

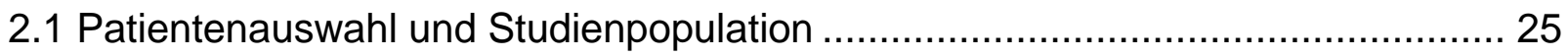

2.2 Durchführung des diagnostischen Mamma-MRT ........................................... 25

2.3 Durchführung der MR-gestreuten Vakuumbiopsien ....................................... 27

2.4 Erhebung der Daten im Rahmen dieser Studie............................................. 28

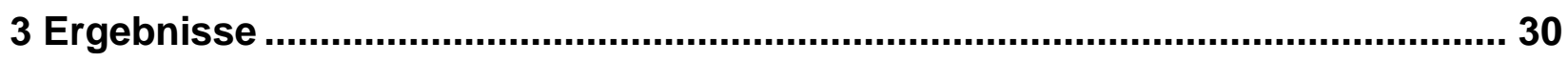

3.1 Charakterisierung der Studienpopulation ...................................................... 30

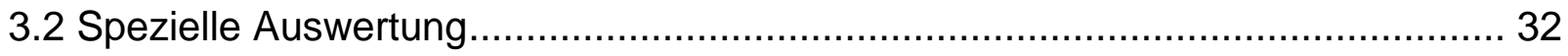

3.2.1 MR-tomografische Läsionsbeurteilung der Foki ....................................... 32

3.2.2 MR-tomografische Läsionsbeurteilung der Herde ...................................... 33 
3.2.3 MR-tomografische Läsionsbeurteilung der NMLs.................................. 41

3.3 Verlaufskontrolle der Studienpopulation ....................................................... 42

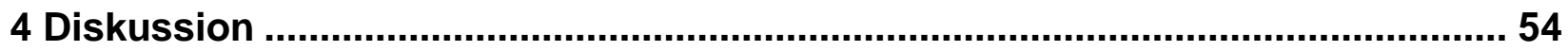

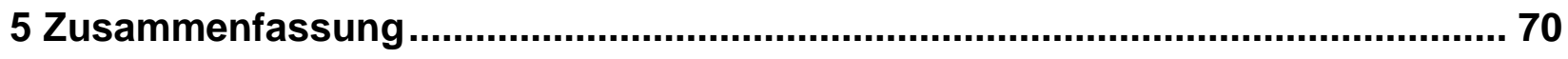

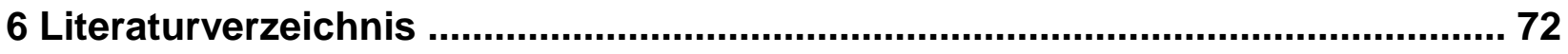




\section{Abbildungsverzeichnis}

Abbildung 1: Inzidenz und Mortalität des Mammakarzinoms im internationalen Vergleich (pro 100.000). Abbildung aus Husmann et al. (2010), Seite 59.

Abbildung 2: Modell der Mehrschrittkarzinogenese des Mammakarzinoms. Abbildung modifiziert nach Beckmann et al. (1997), Seite 162.

Abbildung 3: Dichtedarstellung im Mammogramm: ACR Dichte-Typ I, II, III und IV (von links nach rechts). Abbildung aus Fischer U (2008), Seite 787.

Abbildung 4: Initialer Signalanstieg. Abbildung aus Fischer U (2000), Seite 28. ....................21

Abbildung 5: Postinitialer Signalverlauf. Abbildung aus Fischer U (2000), Seite 29.

Abbildung 6: Prozentuale Verteilung der 3 Formen anreichernder Läsionen im gesamten Patientenkollektiv

Abbildung 7: Anzahl der Foki, Herdbefunde und NML in Abhängigkeit von der MRM-BIRADSKategorie.

Abbildung 8: Fokusgröße in Abhängigkeit von der MRM-BIRADS-Kategorie.

Abbildung 9: Endotumoraler Wassergehalt der Foki in Abhängigkeit zur MRM-BIRADSKategorisierung.

Abbildung 10: Größe des Herdbefundes in Abhängigkeit von der MRM-BIRADS-Kategorie....34

Abbildung 11: Form der Herdbefunde in Abhängigkeit von der MRM-BIRADS-Kategorie.........34

Abbildung 12: Begrenzung der Herde in Abhängigkeit von der MRM-BIRADS.

Abbildung 13: Endotumorale Kontrastmittelverteilung der Herde versus MRM-BIRADSKategorie.

Abbildung 14: Initialer Signalanstieg der Herde in Abhängigkeit von der MRM-BIRADSKategorie.

Abbildung 15: Postinitialer Signalverlauf der Herde in Abhängigkeit von der MRM-BIRADSKategorie.

Abbildung 16: Wassergehalt der Herde in Abhängigkeit von der BIRADS-Kategorie.

Abbildung 17: Endotumorale Septierungen der Herde in Abhängigkeit von der BIRADS-

Kategorie.

Abbildung 18: Prozentuale Verteilung der Punktwerte des Göttingen-Score für die Gesamtzahl aller Herde. 39

Abbildung 19: Prozentuale Verteilung der Gesamtpunkte des Göttingen-Score für die BIRADS3-Kategorie. 
Abbildung 20: Prozentuale Verteilung der Gesamtpunkte des Göttingen-Score für die BIRADS-

4-Kategorie.

Abbildung 21: Größe der NMLs differenziert nach BIRADS-Kategorie.

Abbildung 22: Anreicherungsform der NMLs differenziert nach BIRADS-Kategorie.

Abbildung 23: Dignität der Befunde von 196 Patienten mit Befunden der Kategorie MRM-

BIRADS 3 (23 Befunde histologisch verifiziert).

Abbildung 24: Histologische Klassifikation der Befunde von 198 Patienten mit Befund der Kategorie MRM-BIRADS 4.

Abbildung 25: Verteilung der 3 Typen anreichernder Läsionen in Abhängigkeit von der Dignität des Befundes. 45

Abbildung 26: Fokusgröße in Abhängigkeit von der Dignität der Läsion. 45

Abbildung 27: Größe der Herdbefunde in Abhängigkeit von der Dignität. 46

Abbildung 28: Form der Herdbefunde in Abhängigkeit von der Dignität. 47

Abbildung 29: Begrenzung der Herdbefunde in Abhängigkeit von der Dignität.

Abbildung 30: Endotumorales Verteilungsmuster des Kontrastmittels innerhalb der Herdbefunde in Abhängigkeit von der Dignität. 48

Abbildung 31: Initialer Signalanstieg der Herdbefunde in Abhängigkeit von der Dignität. 49

Abbildung 32: Postinitialer Signalanstieg der Herdbefunde in Abhängigkeit von der Dignität... 49 Abbildung 33: Wassergehalt innerhalb der Herdbefunde in Abhängigkeit von der Dignität. .....50 Abbildung 34: Endotumorale Septierungen der Herdbefunde in Abhängigkeit von der Dignität.

Abbildung 35: Punktwerte des Göttingen-Score der Herdbefunde in Abhängigkeit von der Dignität. .52

Abbildung 36: Größe der NMLs in Abhängigkeit von der Dignität. .53

Abbildung 37: Anreicherungsform der NMLs in Abhängigkeit von der Dignität. .53 


\section{$\underline{\text { Tabellenverzeichnis }}$}

Tabelle 1: Dichtetypisierung des Brustgewebes...................................................... 12

Tabelle 2: Göttingen-Score nach Fischer U. Nach Fischer U (2000) Seite 30......................23 


\section{Abkürzungsverzeichnis}

$\begin{array}{ll}\text { Abb. } & \text { Abbildung } \\ \text { ACR } & \text { American College of Radiology } \\ \text { ADH } & \text { Atypische duktale Hyperplasie } \\ \text { AG } & \text { Arbeitsgemeinschaft } \\ \text { ATEC } & \text { Automated Tissue Excision and Collection } \\ \text { bFGF } & \text { Basic fibroblast growth factor } \\ \text { BIRADS } & \text { Breast Imaging Reporting and Data System } \\ \text { BRCA 1 } & \text { Breast Cancer Gen 1 } \\ \text { BRCA 2 } & \text { Breast Cancer Gen 2 } \\ \text { bzW. } & \text { Beziehungsweise } \\ \text { CC } & \text { Kraniokaudal } \\ \text { cm } & \text { Zentimeter } \\ \text { CUP } & \text { carcinoma unknown primary } \\ \text { 2D } & \text { Zwei dimensional } \\ \text { 3D } & \text { Drei dimensional } \\ \text { d.h. } & \text { Das heist } \\ \text { DCIS } & \text { Duktale(s) carcinoma(ta) in situ } \\ \text { DICOM } & \text { Digital Imaging and Communications in Medicine } \\ \text { DNA } & \text { Desoxyribonukleinsäure } \\ \text { et. al. } & \text { Ft alii (lat. und andere) } \\ \text { Fa. } & \text { Firma } \\ \text { FOV } & \end{array}$




\begin{tabular}{|c|c|}
\hline Gd-DTPA & Gadolinium-Diethylentriaminpentaessigsäure \\
\hline GE & Gradienten-Echo-Sequenz \\
\hline GEKID & Gesellschaft der epidemiologischen Krebsregister in Deutschland \\
\hline ggf. & Gegebenenfalls \\
\hline G-System & Gauge System \\
\hline IDC & Invasiv ductal carcinoma \\
\hline ILC & invasiv lobular carcinoma \\
\hline Inv. Time & Inversionszeit \\
\hline IR & Inversion Recovry \\
\hline i.v. & Intavenös \\
\hline $\mathrm{kg}$ & Kilogramm \\
\hline KM & Kontrastmittel \\
\hline LIN & Lobuläre intraepitheliale Neoplasie \\
\hline $\min$ & Minute \\
\hline $\mathrm{mm}$ & Millimeter \\
\hline $\mathrm{mmol}$ & Millimol \\
\hline MRI & Magnetic resonance imaging \\
\hline MRM & Magnetresonanz-Mammographie \\
\hline MRT & Magnetresonanz-Tomographie \\
\hline ms & Millisekunde \\
\hline$n$ & Anzahl \\
\hline NML & Non-mass-like lesion \\
\hline NOS & Not otherwise specified \\
\hline o.g. & Oben genannte \\
\hline ROI & Region of interest \\
\hline
\end{tabular}




$\begin{array}{ll}\text { S. } & \text { Seite } \\ \text { S3 } & \text { Stufe 3 } \\ \text { sog. } & \text { So genannt } \\ \text { s.u. } & \text { Siehe unten } \\ \text { T } & \text { Tesla } \\ \text { T1 } & \text { Spin- Gitter-Relaxationszeit (Longitudinale Relaxation) } \\ \text { T2 } & \text { Spin-Spin-Relaxationszeit (Quer-Relaxation) } \\ \text { Tab. } & \text { Tabelle } \\ \text { TDLE } & \text { Terminale duktulo-lobuläre Einheit } \\ \text { TE } & \text { Echozeit } \\ \text { TR } & \text { Repetitionszeit } \\ & \\ \text { u.a. } & \text { Unter anderem } \\ \text { US } & \text { Ultraschall } \\ \text { VEGF } & \text { Vascular endothlial growth factor } \\ \text { z.B. } & \text { Zum Beispiel }\end{array}$




\section{Einleitung}

In der bildgebenden Diagnostik des Mammakarzinoms, die sich schwerpunktmäßig auf die Untersuchungsverfahren Mammographie, Sonographie und Mamma-MRT stützt, stößt die Bildinterpretation immer wieder an Grenzen bei Befunden, die entweder kontrolliert oder einer Abklärung durch ein perkutan-bioptisches Verfahren zugeführt werden sollten. Das American College of Radiology (ACR) unterscheidet für die Klassifikation entsprechender Befunde allgemein die Kategorie BIRADS 3 (wahrscheinlich gutartig, Kontrolle empfohlen) und die Kategorie BIRADS 4 (möglicherweise bösartig, histologische Abklärung empfohlen).

Für alle bildgebenden Verfahren ist die Zuordnung von Befunden in der Bildgebung zu einer BIRADS-Kategorie nicht klar definiert. Sie unterliegt neben dem Stellenwert anerkannter Auswertekriterien auch der Intuition und insbesondere dem Erfahrungsstand des Auswerters.

Letztendlich beinhaltet die Differenzierung zwischen Befunden der Kategorie BIRADS 3 und BIRADS 4 die Schwelle zwischen konservativer Vorgehensweise und Abklärung durch minimal-invasive Techniken. Hiermit verbunden ist die Frage der Kosten, die bei vakuumbioptischer Vorgehensweise deutlich höher sind als bei einer nicht-invasiven Kontrolluntersuchung. Für die betroffene Frau stellt die perkutane Biopsie einerseits zwar das invasivere Verfahren dar, andererseits impliziert eine abwartende Strategie mit Kontrolluntersuchungen in 6-Monats-Intervallen aufgrund der unklaren Gesamtkonstellation Ängste, die individuell in unterschiedlicher Ausprägung die Lebensqualität der betreffenden Frau drastisch reduzieren können.

Die Zielsetzung der vorliegenden Studie war es daher, bei Frauen mit einer MammaMRT und Befunden der Kategorien MRM-BIRADS 3 und MRM-BIRADS 4 zu evaluieren, ob es im Rahmen der etablierten Auswertekriterien Befundmuster gibt, die eine zuverlässige Zuordnung zu einer der beiden Vorgehensweisen, also Kontrolle versus perkutane Biopsie, erlauben. Darüber hinaus sollte geklärt werden, ob eventuell sogar Konstellationen nachweisbar sind, die als „zuverlässig gutartig“ (MRM -BIRADS 2) oder 
„Zuverlässig bösartig“ (MRM-BIRADS 5) einzustufen sind, um einerseits auf Kontrollen, andererseits auf perkutan-bioptische Abklärungen gänzlich verzichten und kostensparender agieren zu können. 


\subsection{Epidemiologie des Mammakarzinoms}

Mit über einer Millionen Neuerkrankungen pro Jahr ist das Mammakarzinom der häufigste bösartige Tumor der Frau weltweit (Giersiepen et al. 2005). In der Statistik der Gesellschaft der epidemiologischen Krebsregister (GEKID) beträgt die Inzidenz des Mammakarzinoms 29,3\% aller Krebsneuerkrankungen (Husmann et al. 2010). Es wird vermutet, dass etwa 10\% der weiblichen Bevölkerung im Laufe ihres Lebens an Brustkrebs erkrankt. (Claus et al. 1991). Bezüglich der Mortalität ist das Mammakarzinom mit 18\% die häufigste Krebstodesursache (Giersiepen et al. 2005). Abbildung 1 verdeutlicht, dass Deutschland bei der Mortalität sowie bei der Inzidenz des Mammakarzinomes im internationalen Vergleich eine mittlere Position einnimmt (Husmann et al. 2010).

Ein erhöhtes Risiko für die Entstehung von Brustkrebs zeigt sich bei Frauen, in deren naher Verwandtschaft ein Mammakarzinom aufgetreten ist. Des Weiteren stellen laut S3-Leitline zur Brustkrebsfrüherkennung eine Krebserkrankung als Kind, frühe Menarche, erste Entbindung nach dem 40. Lebensjahr, Hormonzusatztherapie während und nach der Menopause, kurzes bzw. kein Stillen sowie Alkoholkonsum und Rauchen ein erhöhtes Brustkrebsrisiko dar (Ortmann et al. 2008).

Seit den 1990er Jahren sinkt die Brustkrebssterblichkeit jedoch, was vor allem auf die Früherkennung und die Verbesserung der Therapie zurückzuführen ist (Becker 2001). Des Weiteren ist seit wenigen Jahren auch die Inzidenz gering fallend, was u.a. als ein Effekt der nachlassenden Hormonsubstitution in den Wechseljahren angesehen wird (Urdl et al. 1996). 


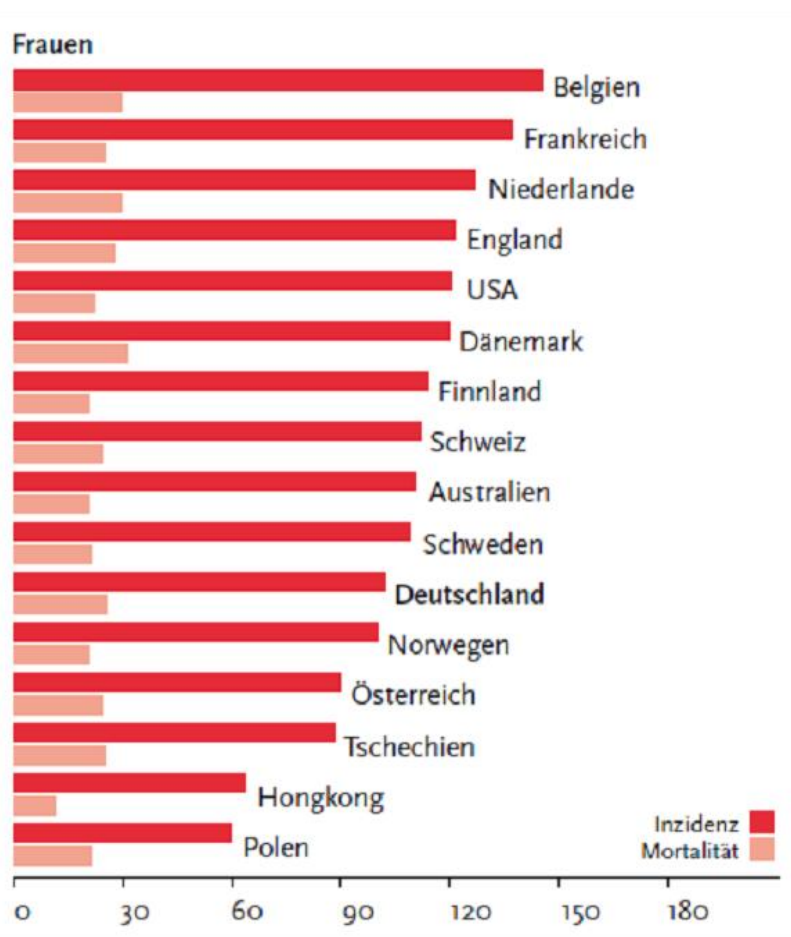

Abbildung 1: Inzidenz und Mortalität des Mammakarzinoms im internationalen Vergleich (pro 100.000). Abbildung aus Husmann et al. (2010), Seite 59.

\subsection{Anatomie der Brustdrüse}

Die weibliche Brustdrüse besteht aus 15-20 Drüsenlappen (Lobi), deren an der Mamille endender Ausführungsgang (Ductus lactifercolligens) sich bis zu den sekretorischen Einheiten, den terminalen duktulo-lobulären Einheiten (TDLE), verzweigt. Jede dieser Einzeldrüsen wird von einem lockeren Bindegewebsmantel bekleidet und durch straffes septenartiges Bindegewebe von den Nachbardrüsen isoliert. Eine gesonderte Stellung kommt der TDLE bei der Karzinogenese zu. Sie ist der Ursprung der meisten pathologischen Veränderungen der Mamma (Leonhardt 1985; Wittekind 2004).

\subsection{Grundlagen der Karzinogenese}

Bedingungen für die Entwicklung eines malignen Geschehens sind Mutationen in einzelnen oder mehreren Zellen. Alles in allem sind acht bis zehn Mutationen nötig um eine Zelllinie bösartig entarten zu lassen (Lewin 1998). Wenn diese Mutationen nicht 
durch die DNA-Reparaturmechanismen oder den programmierten Zelltod (Apoptose) beseitigt werden, so persistieren sie und sind irreversibel (Horn 2003).

Durch moderne molekularbiologische Techniken werden immer neue Einsichten in die molekulargenetischen Mechanismen der Karzinomentstehung und -progression gewonnen. Das Modell der so genannten Mehrschrittkarzinogenese wird in der Abbildung 2 anschaulich dargestellt. Hier wird die Karzinogenese ausgehend von der normalen Epithelzelle, über physiologische und unphysiologische Zellproliferationen bis zum Carcinoma in situ und dann zum invasiven Karzinom mit zirkulierenden Tumorzellen und Fernmetastasen beschrieben. Der Entwurf integriert unterschiedlichste Faktoren, deren Interaktion, sowie zeitliche Abfolge weitestgehend unbekannt ist (Beckmann et al. 1997).

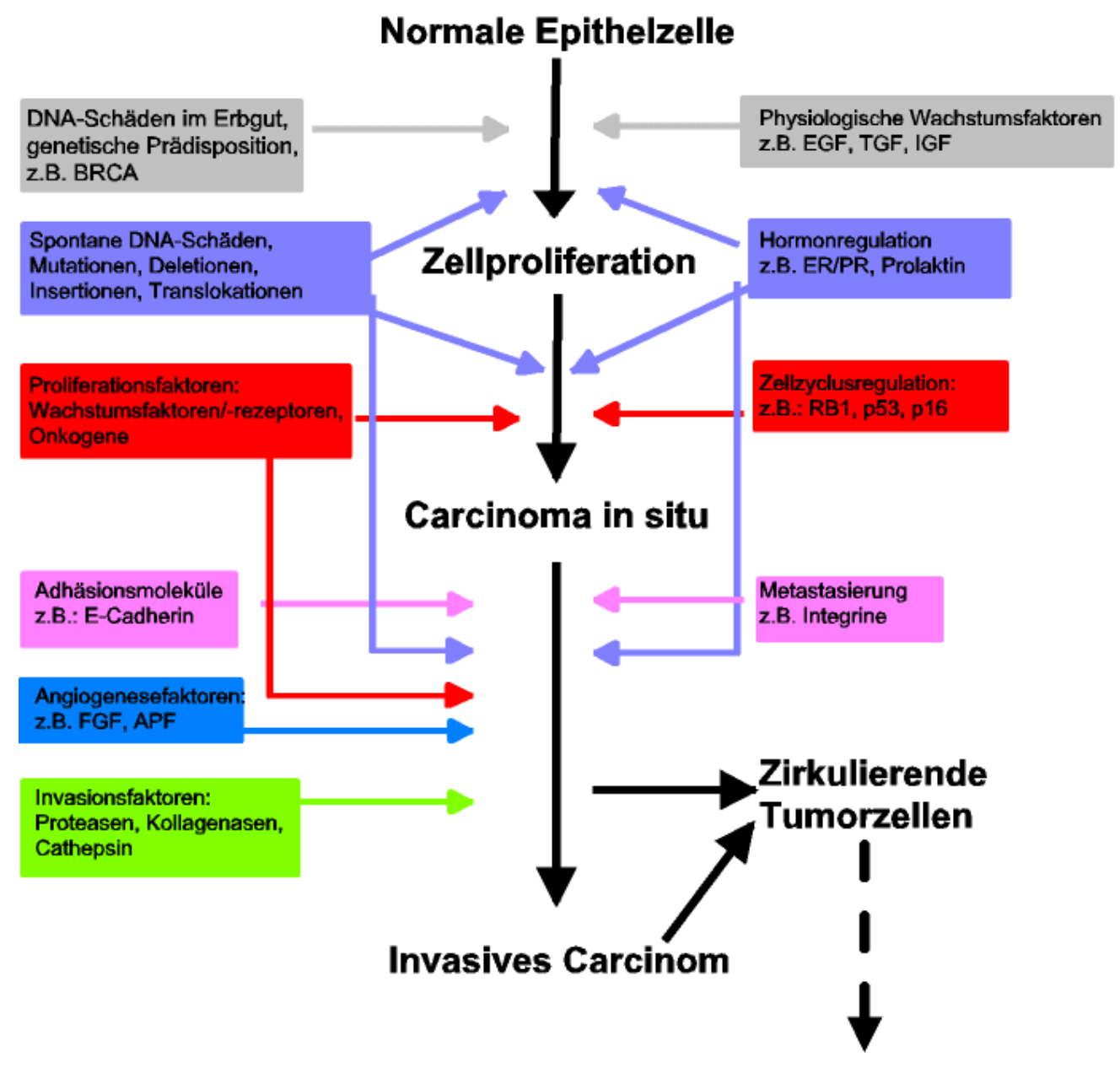

Fernmetastasen

Abbildung 2: Modell der Mehrschrittkarzinogenese des Mammakarzinoms. Abbildung modifiziert nach Beckmann et al. (1997), Seite 162. 
Anhand der Mehrschrittkarzinogenese ist zu erkennen, dass einzelne Mutationen für den menschlichen Organismus noch keine Probleme darstellen. Erst wenn es zu der Aktivierung von Proto-Onkogenen oder zur Inaktivierung von Tumorsuppressorgenen kommt und sich in der Folge eine Zelle ungehindert teilt (Verlust der Kontaktinhibitation), kann sich ein Tumor entwickeln. Proto-Onkogene sind solche Gene, die in der Lage sind, das Wachstum einer Zelle und damit verbundene Zellabläufe positiv zu regulieren. Hierzu zählen Wachstumsfaktoren, zytoplasmatische Übermittlerproteine oder auch Transkriptionsfaktoren. Tumorsupressorgene hingegen wirken zumeist inhibierend auf das Zellwachstum. Des Weiteren kontrollieren sie die Transkription und den Zellzyklus oder induzieren die Apoptose. Fällt dieser Kontrollmechanismus weg, kommt es zur unphysiologischen Proliferation und somit zum malignen Wachstum der Zelle. Diese Zellen können sich dann in einem Organ ungehindert ausbreiten, ohne andere anatomische Strukturen zu berücksichtigen (Beckmann et al. 1997; Horn 2003).

Unter die Gruppe der Tumorsupressorgene fallen die Gene BRCA 1 und BRCA 2, (Breast Cancer), die bei der Kontrolle der DNA-Reparatur, der Transkription und des Zellzyklus mitwirken. Eine Entartung dieser Gene führt für die betroffene Frau zu einer drastischen Steigerung des Risikos für die Entstehung eines Mammakarzinoms (Beckmann et al. 1997; Horn 2003).

\subsubsection{Tumorangiogenese}

Vor mehr als 30 Jahren revolutionierte Judah Folkmann die Denkweise der Tumorbiologie. Er stellte fest, dass die Neubildung von Gefäßen (Neoangiogenese) in Tumoren für das Wachstum und das Überleben selbiger von großer Bedeutung ist (Folkman 1971). Die Neoangiogenese ist allerdings nicht nur für die Entwicklung und das Wachstum des Primärtumors notwendig. Sie erhöht auch die Wahrscheinlichkeit einer hämatologischen Metastasierung, da der Tumor hierdurch Zugang zum gesamten Gefäßsystem des Wirtes erhält (Fidler und Ellis 1994; Yancopoulos et al. 2000). Die hämatologische Tumorstreuung wird dadurch gefördert, dass die neu gebildeten Gefäße durchlässige Basalmembranen besitzen, die die Penetration von Tumorzellen (oder 
auch Kontrastmittel) erleichtern (Brinck 2000). Die beiden Wachstumsfaktoren Fibroblastenwachstumsfaktor (bFGF) und der vaskuläre endotheliale Wachstumsfaktor (VEGF) spielen bei der Neoangiogenese eine bedeutende Rolle. Beide stimulieren die Migration und Proliferation von Endothelzellen und unterstützen somit den Prozess der Gefäßneubildung (D'Amore und Thompson 1987; Brinck 2000). Das zur Gruppe der vaskulärenendothelialen Wachstumsfaktoren gehörende angiogene VEGF regt beispielsweise in der Region des Tumors das Wachstum neuer, aus Endothelzellen aufgebauter tubulärer Gefäßstrukturen an, die die Vorstufe von Tumorblutgefäßen darstellen (Barleon et al. 1997).

\subsection{Maligne Erkrankungen der Brustdrüse}

Unter dem Begriff Mammakarzinom werden maligne Tumoren zusammengefasst, die von den Epithelien der Milchgänge (duktale Karzinome) oder von den lobulären Drüsenendstücken (lobuläre Karzinome) ausgehen. Hierbei werden die nicht invasiven (DCIS, duktales Carcinoma in situ) von den invasiven Formen abgegrenzt. Bei der präinvasiven Form ist die Karzinomentstehung und -ausbreitung auf das Epithel der Lumina der TDLE beziehungsweise auf die lobulären Zelllinien, die den Drüsenläppchen entstammen, beschränkt (Wittekind 2004). Hier kommt es auf Grund der schlechten Nahrungsversorgung und beschleunigten Zellteilung in dem intraduktalen Stadium zu Nekrosen. Dieser Gewebszerfall wird von entzündlichen Reaktionen begleitet, in deren Verlauf es zur Ausbildung feinster Verkalkungen kommen kann. Im Sinne einer Früherkennung ist es möglich, diese Mikroverkalkungen unter Zuhilfenahme von bildgebenden Verfahren (Mammographie) zuverlässig nachzuweisen (Lanyi 1982).

Bei dem invasiven Karzinom hingegen haben die malignen Zellen die Basalmembran durchwachsen. Konsekutiv können sie somit lymphogen und hämatogen metastasieren. Bei der lymphogenen Metastasierung sind vor allem die axillären Lymphknoten betroffen. Hämatogen dominieren Metastasierungen in Knochen mit 60\%, Lunge (60\%) und Leber (50\%) (Wittekind 2004). 


\subsubsection{Duktales Carcinoma in situ (DCIS)}

Obwohl nicht alle DCIS-Tumoren invasiv werden, wird die Gruppe der DCIS-Tumore als Präkanzerose angesehen, denn in 30-50\% der Fälle ist mit der Entwicklung eines invasiven Karzinoms zu rechnen (Frykberg und Bland 1994; Böcker et al. 2001).

Das duktale Carcinoma in situ (DCIS) ist gekennzeichnet durch eine Zellproliferation von malignen epithelialen Zellen im Milchgangsystem, welche vom gefäßführenden Stroma durch eine intakte Basalmembran abgegrenzt sind (Böcker et al. 2001). Lymphogene oder hämatogene Metastasierungen können somit bei einem DCIS-Tumor nicht auftreten (Wittekind 2004).

Hinsichtlich histopathologischer Kriterien stellen die DCIS-Tumoren eine heterogene Gruppe dar. Histologisch unterscheidet man den Komedotyp, der zentrale Nekrosen aufweist, sowie den sogenannten Non-Komedotyp (solide, cribriform, mikropapillär, papillär und clinging). Häufig treten Mischformen auf (Tavassoli et al. 2003). Um eine morphologisch einfache und reproduzierbare Beurteilung zu gewährleisten, stellen 1995 Silverstein et al. eine neue prognostische Einteilung des DCIS, die Van-NuysKlassifikation, vor. Diese Klassifikation stützt sich lediglich auf die morphologischen Kriterien „Kerngrading“ und „Komedo-Nekrosen“. Es wird unterschieden zwischen einem high-grade-DCIS-Tumor (große Zellkerne und deutliche Zellkernpolymorphie), einem intermediate type des DCIS (monomorphes Erscheinungsbild, Zellen mit kleinem Zellkern sowie intraluminale Nekrosen) und den non-high-grade-DCIS-Tumoren (monomorphes Erscheinungsbild mir kleinen Zellkernen, aber keinen Nekrosen) (Silverstein et al. 1995; Allred und Mohsin 2000). High-grade-DCIS zeigen höhere Raten einer Mikroinvasion und eine höhere Wahrscheinlichkeit eines Rezidivs als non-highgrade DCIS, so dass sie die ungünstigste Prognose in der Gruppe der DCIS-Tumoren aufweisen (Tavassoli et al. 2003).

Klinisch weisen DCIS-Tumoren oft kein Korrelat auf und nur in 10\% der Fälle liegt ein Tastbefund vor. Im Einzelfall kann es zur pathologischen Sekretion oder Mamillenveränderung kommen. Von großer Relevanz für die frühe Erkennung von DCIS-Tumoren sind die bereits angesprochenen, in der Mammographie nachweisbaren 
Mikroverkalkungen sowie lineare und dendritische Mehranreicherungen in der hochaufgelösten Mamma-MRT (Lanyi 1982; Fischer U 2000; Kuhl et al. 2000).

\subsubsection{Invasives Mammakarzinom}

\subsubsection{Invasiv duktales Mammakarzinom (IDC)}

Mit circa $80 \%$ stellt das invasiv duktale Karzinom den häufigsten histologischen Subtyp der invasiven Karzinome der weiblichen Brust dar (Wittekind 2004). Da sich der IDC aufgrund fehlender spezieller Zelldifferenzierung keiner anderen Kategorie invasiver Mammakarzinome zuordnen lässt, wird es auch als not otherwise specified („NOS“) bezeichnet. Ausgehend vom Epithel der terminalen Milchgänge ahmen die Tumorzellen entweder Drüsenschläuche nach oder bilden solide Zellbalken. Es kommt zur Überschreitung der Basalmembran, was eine mannigfache Stromabildung induziert (Wittekind 2004). Morphologisch können unregelmäßig begrenzte Tumoren mit sternförmigen Ausläufern in das umliegende Fettgewebe von umschriebenen Konfigurationen abgegrenzt werden. Diese verschiedenen Wachstumsformen prägen das Erscheinungsbild des IDC in der bildgebenden Diagnostik in Form von raumfordernden Veränderungen mit unscharfer Begrenzung, potentiell assoziierten Mikroverkalkungen sowie obligat gesteigertem Durchblutungsmuster (Buchberger et al. 1999; Fischer U 2000; Heywang-Köbrunner et al. 2008).

\subsubsection{Invasiv lobuläres Mammakarzinom (ILC)}

Mit etwa $10 \%$ stellt das ILC die zweithäufigste Variante der invasiven Mammakarzinome dar. Histologisch imponieren entweder einreihige Tumorzellstränge, die von opulentem Stroma umgeben sind und diffus in selbiges infiltrieren (Indian filepattern) oder Tumorzellen, die zirkulär einzelne Milchgänge umspannen (targetoid pattern) (Jayaram et al. 2000; Böcker et al. 2001). Aufgrund des oftmals zerstreuten Wachstums und der sporadisch abnormen Metastasierung (abdominell, pelvin), ist die Prognose im 
Vergleich zum duktalen Karzinom als schlechter einzuschätzen. Typischerweise treten keine Verkalkungen auf (Fischer U 2000; Wittekind 2004; Heywang-Köbrunner et al. 2008).

\subsection{Mammadiagnostik}

Die Diagnostik der weiblichen Brust stützt sich auf die klinische Untersuchung und die Darstellung der Brustdrüsenstrukturen mittels bildgebender Verfahren. Bisher wurden die meisten Tumoren in Deutschland durch die Patientin selbst oder den behandelnden Arzt entdeckt (Schleicher und Ammon 1998). Hierbei handelt es sich jedoch nicht um eine Früherkennung von Brustkrebs, da die durchschnittliche Tumorgröße des palpatorisch erfassten Mammakarzinoms zwischen 2 und $3 \mathrm{~cm}$ liegt. Früherkennung mit der Konsequenz einer sehr guten Prognose sieht allerdings die Detektion von Brustkrebs im Stadium DCIS oder bis zu einer Größe von $10 \mathrm{~mm}$ bei invasiven Tumorformen vor. Die Erkennung solcher „frühen“ bzw. „kleinen“ Karzinome macht üblicherweise den Einsatz bildgebender Untersuchungsverfahren notwendig. Hier kommt der Mammographie durch den zuverlässigen Nachweis von Mikroverkalkungen eine große Bedeutung zu (Müller-Schimpfle 2008). Bei Frauen mit hohem Lebenszeitrisiko kommt zudem dem Einsatz der Mamma-MRT eine übergeordnete Bedeutung zu (Kuhl et al. 2010).

\subsubsection{Klinische Untersuchung der Mamma}

Zur klinischen Untersuchung der Mamma gehören die Inspektion und die Palpation. Bei der Inspektion wird die Haut, Brustkontur sowie der Mamillen- Areolakomplex sowohl bei erhobenen als auch gesenkten Armen untersucht. Fokussiert werden sollte sich auf Retraktionen, Verfärbungen, Verdickungen, Infiltrationen, Hautanhangsgebilde, eingezogene Mamillen sowie ekzematöse Veränderungen der Mamillenhaut (Kettritz 2007). 
Die Palpation wird im Sitzen oder Liegen unter Einbeziehung der regionalen Lymphabflussgebiete durchgeführt. Verhärtungen oder Knoten sollten auf Größe, Konsistenz, Verschieblichkeit sowie Schmerzhaftigkeit untersucht werden (Kettritz 2007). Laut S3-Leitlinie zur Brustkrebsfrüherkennung ist die alleinige BrustSelbstuntersuchung allerdings nicht in der Lage, die Brustkrebssterblichkeit zu senken (Albert und Naß-Griegoleit 2008).

\subsubsection{Bildgebende Diagnostik}

\subsubsection{Mammographie}

Die Mammographie gilt seit Jahrzehnten als das wichtigste bildgebende Verfahren in der Diagnostik des Mammakarzinoms (Friedrich 1993). Die Bedeutung der Röntgenmammographie liegt in erster Linie in der Erkennung klinisch okkulter Karzinome und präinvasiver Brustkrebsvorstufen durch die zuverlässige Darstellung kleinster Mikrokalzifikationen. Die Detektion solcher feinster Mikroverkalkungen wird durch das Ausmaß der Parenchymdichte im Mammogramm nicht beeinflusst. Beim DCIS gelten gruppierte Mikroverkalkungen als das häufigste Leitsymptom der Diagnose im Mammogramm (Tulusan et al. 2005). Werden diese unter Zuhilfenahme der Mammographie früh genug entdeckt und operativ entfernt, so hat das DCIS eine exzellente Langzeitprognose. Bei den invasiven Karzinomen, die zu etwa 30\% mit Mikroverkalkungen assoziiert sind, korrelieren die Heilungschancen mit der Tumorgröße und den Lymphknotenmetastasen bei Diagnosestellung (Tabar et al. 2000).

Die Sensitivität der Mammographie wird für Mammakarzinome, die nicht mit Mikroverkalkungen einhergehen, essentiell von der Parenchymdichte im Mammogramm und hiermit verbunden, natürlich von der Altersgruppe der untersuchten Frauen beeinflusst (Kerlikowske et al. 1996; Rosenberg et al. 1998; Mandelson et al. 2000). Bei guten technischen Voraussetzungen liegt die Sensitivität bei Patientinnen im Alter von 40-49 Jahren zwischen 62-77\%, bei Frauen älter als 65 Jahre bei 81-91\% (Tabar et al. 1992; Rosenberg et al. 1998; Olivotto et al. 2000). Einen noch größeren Einfluss scheint 
die Gewebsdichte der Brust zu haben. Die Sensitivität liegt bei einer Brust mit sehr dichtem Gewebe (Dichtetyp III, IV gemäß American College of Radiology) zwischen 30 $68 \%$ und bei $85-90 \%$ bei einer fettreichen Brust gemäß Dichtetyp I nach ACR (Rosenberg et al. 1998; Mandelson et al. 2000; Pisano et al. 2005; Boyd et al. 2007). Um diese Dichteabhängigkeit zu berücksichtigen und somit einen Anhalt für die Sensitivität eines Mammogramms zu erhalten, hat das American College of Radiology (ACR) eine Dichtetypisierung erstellt. Sie ist abhängig vom Anteil des Drüsengewebes der Brust im Mammogramm (American College of Radiology 1993) (Tab.1).

\begin{tabular}{|l|l|c|}
\multicolumn{1}{|c|}{ Dichtetypisierung } & \multicolumn{1}{c|}{ Gewebe } & Parenchymanteil \\
\hline Typ I: & überwiegend lipomatös & $<25 \%$ \\
\hline Typ II: & fibroglandulär & $25-50 \%$ \\
\hline Typ III: & inhomogen dicht & $50-75 \%$ \\
\hline Typ IV: & sehr dicht & $>75 \%$ \\
\hline
\end{tabular}

Tabelle 1: Dichtetypisierung des Brustgewebes.

Diese Klassifikation des Dichtetyps im Mammogramm erlaubt letztendlich eine Aussage zur Zuverlässigkeit der individuell angefertigten Mammographie, die in Abhängigkeit vom Ausmaß des Drüsenkörpers von 30\% (Typ IV) bis 98\% (Typ I) reichen kann (Fischer U und Helbich 2006; Fischer U 2008). 


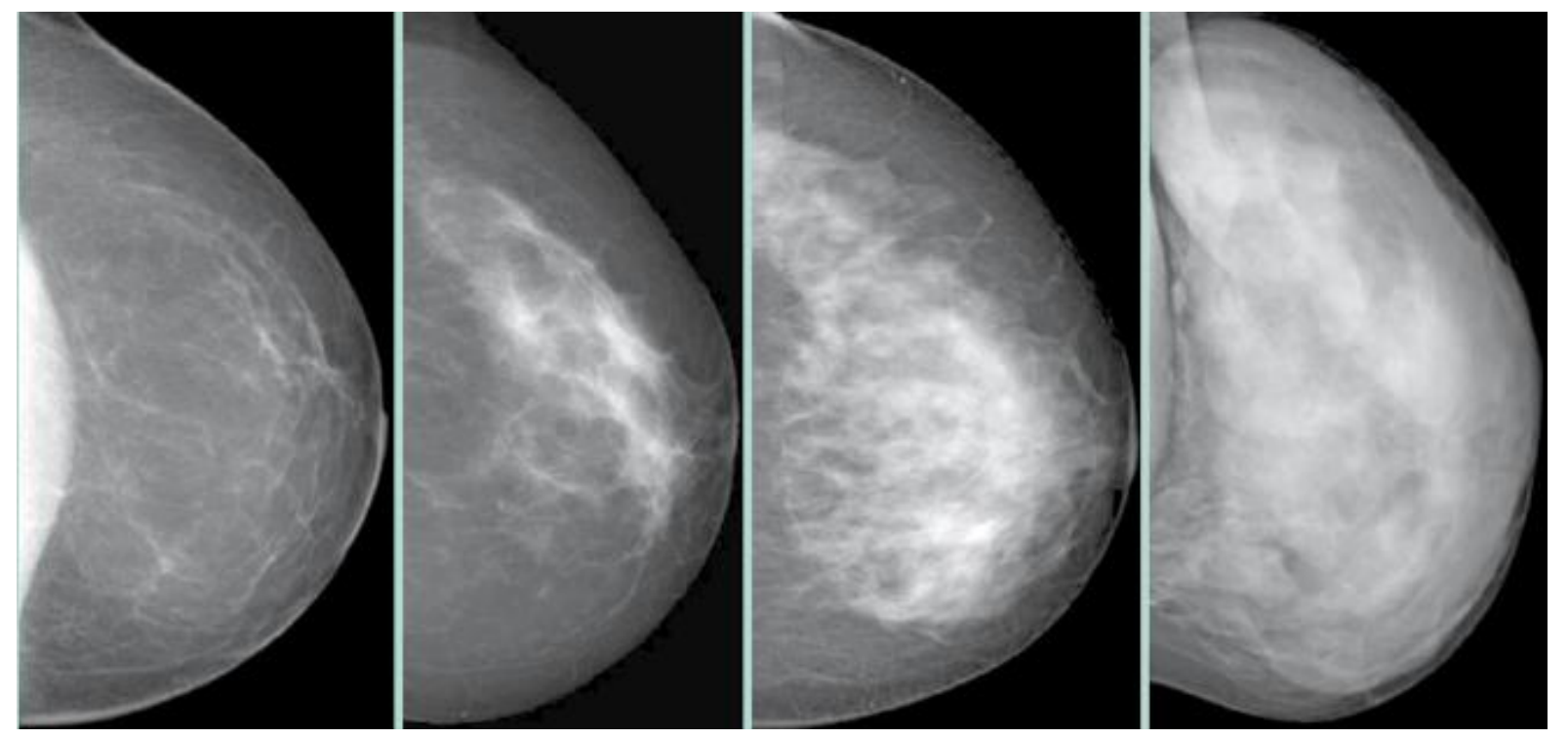

Abbildung 3: Dichtedarstellung im Mammogramm: ACR Dichte-Typ I, II, III und IV (von links nach rechts). Abbildung aus Fischer U (2008), Seite 787.

Typische Indikationen für die Durchführung einer Mammographie umfassen laut S3Leitlinie zur Brustkrebsfrüherkennung u.a.: Klinisch auf Brustkrebs hinweisende Symptome (z.B. Tastbefund, Hautveränderung, pathologische Sekretion), Zustand nach invasivem/non-invasivem Mammakarzinom, familiäre Prädisposition, Frauen mit Hochrisiko-Profil, positiver Sonographie-Befund, entzündliche Veränderungen sowie Früherkennungsuntersuchungen im Sinne eines Mammographie-Screenings (Schreer et al. 2008).

\subsection{Befundbewertung - BI-RADS ${ }^{\mathrm{TM}}$-Klassifikation}

Zur Vereinfachung und Standardisierung mammographischer Befunde wurde im Jahre 1993 von der entsprechenden Arbeitsgruppe der Amerikanischen Röntgengesellschaft (American College of Radiology) das „Breast Imaging Reporting and Data System“ (BIRADS $^{\text {TM}}$ ) erarbeitet (American College of Radiology 1993). Die mittlerweile 4. Ausgabe aus dem Jahr 2004 umfasst neben der Mammographie auch die entsprechenden Vorgaben zur Mammasonographie und Mamma-MRT. Das „BIRADS“, wie es inzwischen im deutschsprachigen Raum kurz genannt wird, gilt mittlerweile als 
internationaler Leitfaden für die Terminologie und Befundklassifizierung in der bildgebenden Mammadiagnostik (American College of Radiology 2004).

Im ersten Teil des BIRADS, dem „Breast Imaging Lexicon“, wird die Nomenklatur der drei Hauptbefunde (Herd, Verkalkung, Architekturstörung) sowie anderer mammographischer Auffälligkeiten präsentiert. Dies zielt auf eine normierte und klare Terminologie der sich darstellenden Befunde ab.

Herdbefunde werden hinsichtlich ihrer Form (rund, gelappt, oval, unregelmäßig), ihrer Ränder (glatt begrenzt, unscharf, mikrolobuliert, spikuliert) und ihrer Dichte (hypodens, isodens, hyperdens, Fett enthaltend) im Vergleich zum restlichen Parenchym unterschieden. Bei Verkalkungen unterscheidet man prinzipiell ihre Größe, Konfiguration (monomorph, amorph, pleomorph) und ihr Verteilungsmuster (gruppiert, linear, segmental, regional, diffus). Die Architekturstörung ist per se durch eine regional gestörte Gewebetexturstörung charakterisiert.

Im zweiten Teil des BIRADS wird eine Kategorisierung der vorliegenden Befunde vorgestellt. Unterschieden werden im Kern der Sache 5 BIRADS-Stufen (BIRADS 1 bis 5), die eine Aussage zur Wahrscheinlichkeit für das Vorliegen eines Mammakarzinoms zum Ausdruck bringen. Hiermit verbunden werden auch klare Empfehlungen formuliert, welche Konsequenzen aus der jeweiligen Kategorisierung gezogen werden sollten. Weitere Empfehlungen betreffen die Kategorie BIRADS 0, die zum Ausdruck bringt, dass zur abschließenden Beurteilung der Mammographie zusätzliche Untersuchungen notwendig sind und die Kategorie BIRADS 6, die im Falle eines perkutan-bioptisch gesicherten Mammakarzinoms zu wählen ist:

\section{BIRADS Kategorie 0:}

Mammogramm ist nicht abschließend $z u$ beurteilen. Weitere Bildgebung (z.B. Sonographie, Tubuskompressionsaufnahmen, Vergrößerungsmammographie) ist erforderlich. 


\section{BIRADS Kategorie 1:}

Mammogramm ist abschließend $z u$ beurteilen. Es liegt kein relevanter Befund im Mammogramm vor. Die Mammae sind symmetrisch. Es finden sich keine Herdbefunde, architektonischen Störungen oder suspekte Verkalkungen. Karzinomrisiko 0\%.

\section{BIRADS Kategorie 2:}

Mammogramm ist abschließend $\mathrm{zu}$ beurteilen. Die Mammographie ist unauffällig, allerdings liegt ein beschreibenswerter Befund vor, der aufgrund seiner Morphologie eindeutig als benigne einzustufen ist (z.B. Lipom, Ölzyste, verkalktes Fibroadenom). Karzinomrisiko $0 \%$.

\section{BIRADS Kategorie 3:}

Mammogramm ist abschließend $\mathrm{zu}$ beurteilen. Es zeigt sich eine Auffälligkeit im Mammogramm, die mit hoher Wahrscheinlichkeit benigne ist. Karzinomrisiko $<2 \%$. Es wird eine kurzfristige Verlaufskontrolle, üblicherweise nach 6 Monaten, empfohlen.

\section{BIRADS Kategorie 4:}

Mammogramm ist abschließend $\mathrm{zu}$ beurteilen. Es findet sich im Mammogramm ein suspekter Befund, der jedoch nicht die typischen Charakteristika eines Malignoms aufweist. Karzinomrisiko 2-90\%. Eine Unterteilung in die Kategorien BIRADS 4a (Risiko $2-30 \%)$, BIRADS 4b (30-60\%) und BIRADS 4c (60-90\%) ist möglich. Eine histologische Abklärung des Befundes, bevorzugt durch eine perkutane Biopsie, wird empfohlen.

\section{BIRADS Kategorie 5:}

Mammogramm ist abschließend zu beurteilen. Es besteht der hochgradige Verdacht auf ein Malignom, da die typischen Kriterien eines Karzinoms erfüllt sind. Karzinomrisiko $>90 \%$. Der Befund stellt eine Indikation für eine histologische Abklärung dar, damit anschließend adäquate therapeutische Maßnahmen eingeleitet werden können. 


\section{BIRADS Kategorie 6:}

Die Malignität einer Auffälligkeit im Mammogramm wurde histologisch nach einer perkutanen Biopsie gesichert. Geeignete onkologische Maßnahmen werden erforderlich.

\subsubsection{Mammasonographie}

Als Ergänzung zur Mammographie hat sich die Ultraschalluntersuchung zur Abklärung palpabler oder mammographisch unklarer Herdbefunde seit langem etabliert. Als alleinige Methodik zur Früherkennung gilt sie allerdings als ungeeignet (Duda et al. 2008). Aufgrund der räumlichen Auflösung der Methode ist die Sonographie nicht in der Lage, Mikrokalzifikationen, die Hinweis auf ein malignes Wachstum (DCIS) sein können, zuverlässig zu erfassen (Kolb et al. 1998; Buchberger et al. 1999). Für Frauen unter 40 Jahren ohne Hochrisikoprofil stellt die Mammasonographie allerdings das Verfahren der Wahl dar, da es nicht mit einer Exposition ionisierender Strahlung behaftet ist.

Der additive Einsatz der Sonographie bringt eine Verbesserung der Ergebnisse im Vergleich zur alleinigen Mammographie mit sich. Kolb et al. konnten in Jahre 2002 zeigen, dass durch die Hinzunahme der Sonographie die Sensitivität für Brustmalignome um knapp 20\% angehoben werden konnte (Kolb et al. 2002). In der von Kuhl et al. durchgeführten EVA-Studie betrug die Verbesserung sogar 38\% (Kuhl et al. 2010).

In den S3-Leitlinien werden folgende Indikationen für eine Mammasonographie genannt: Zystendiagnostik, Abklärung unklarer Tastbefunde, komplementäre Beurteilung mammographisch sichtbarer, schwer abgrenzbarer Herdbefunde sowie ergänzender Einsatz bei dichten Parenchymstrukturen. Zudem die Klärung unklarer Tastbefunde bei jungen Frauen in Schwangerschaft und Laktationsperiode als erste bildgebende Modalität, die additive Diagnostik bei Befunden der Kategorie BIRADS 4 und 5 Befunden im Mammogramm und zur Steuerung und Unterstützung interventioneller Maßnahmen (Kettritz 2007; Duda et al. 2008). 
Wie bei der mammographischen Diagnostik, so unterliegt auch die Aussagekraft der Ultraschalluntersuchung der Zusammensetzung des Brustparenchyms. Aus diesem Grund wurden die Dichteklassifikationen gemäß der ACR Einteilung 1-4 entsprechend für die Sonographie übernommen (Kolb et al. 2002).

In Übereinstimmung zur Beurteilung von Mammogramms wird auch bei der Mammasonographie die BIRADS-Klassifizierung des American College of Radiology angewandt (Madjar et al. 2006). Sie sieht folgende Beschreibungen vor:

\section{US-BIRADS Kategorie 0:}

Suffiziente Abklärung und Einschätzung nicht möglich. Alternative bildgebende Abklärung wird empfohlen.

\section{US-BIRADS Kategorie 1:}

Unauffälliger Mammasonographiebefund. Keine Auffälligkeiten sonographisch nachweisbar. Kontrolle in üblichen Intervallen wird empfohlen.

\section{US-BIRADS Kategorie 2:}

Sonographisch eindeutig als gutartig einzustufender Befund (Narben, Zysten, Lymphkonten, Fibroadenome). Kontrolle in üblichen Intervallen wird empfohlen.

\section{US-BIRADS Kategorie 3:}

Auffälligkeit im Ultraschall, die mit hoher Wahrscheinlichkeit benigne ist. Karzinomrisiko $<2 \%$. Es wird eine kurzfristige Verlaufskontrolle, üblicherweise nach 6 Monaten, empfohlen.

\section{US-BIRADS Kategorie 4:}

Suspekter Befund, z.B. Herdbefund zeigt nicht die üblichen Benignitätskriterien (Malignitätswahrscheinlichkeit: 40\%). Eine histologische Abklärung des Befundes, bevorzugt durch eine perkutane Biopsie, wird empfohlen. 


\section{US-BIRADS Kategorie 5:}

Es liegt ein hochgradig suspekter Befund vor, da typische sonographische Malignitätskriterien vorhanden sind. Karzinomrisiko um 95\%. Der Befund stellt eine Indikation für eine histologische Abklärung dar, damit anschließend adäquate therapeutische Maßnahmen eingeleitet werden können.

\section{BIRADS Kategorie 6:}

Histologisch gesicherte Malignität. Geeignete onkologische Maßnahmen erforderlich.

\subsubsection{Mamma-MRT (MRM)}

Die Kernspintomographie der Brust wird seit etwa 35 Jahren in Deutschland eingesetzt, wobei die paramagnetischen Kontrastmittel, die einen entscheidenden Fortschritt sowohl hinsichtlich der Malignomdetektion als auch der Differenzierung von Läsionen darstellten, erst in den 1980er Jahren eingeführt wurden (Heywang et al. 1986; Kaiser und Zeitler 1986; Orel et al. 2006). Die insbesondere bei malignen Tumoren gesteigerte Tumorangiogenese und somit verstärkte Vaskularisation kann durch eine geeignete Kontrastmittelgabe in der Mamma-MRT visualisiert und ausgewertet werden. Sie ist der Hauptgrund für die hohe Sensitivität der MRM bei präinvasiven und invasiven Tumoren. Mit der Optimierung technischer und methodischer Komponenten entwickelte sich die Mamma-MRT bei Verwendung offener Messspulen, geeigneter Kompressorien und hochaufgelöster Untersuchungsprotokolle in den letzten Jahren zum sensitivsten Untersuchungsverfahren im Vergleich zu Mammographie und Sonographie (Berg et al. 2004; Sardanelli et al. 2004; Kuhl et al. 2010). Die bessere Sensitivität der MRT konnte inzwischen auch für DCIS-Tumoren mit hohem Grading nachgewiesen werden (Berg et al. 2004; Kuhl et al. 2007). Der lange Zeit geäußerten Kritik an der geringen Spezifität der Mamma-MRT wurde durch den Einsatz multimodaler Evaluationskonzepte begegnet, die inzwischen vergleichbare Werte der MRT verglichen mit denen der Mammographie und Sonographie erlauben (Kuhl et al. 2010). 
Das Spektrum der MR-Indikationen reicht von dem präoperativen Staging bei Frauen mit Mammakarzinom, der Differenzierung zwischen einem Rezidiv nach brusterhaltender Karzinomtherapie und einer Narbe, der Primärtumorsuche beim CUPSyndrom bis hin zum Einsatz der Mamma-MRT als Problemlöser bei unklarem Befund in Mammographie und/oder Sonographie oder dem primären Einsatz des Verfahrens bei Frauen mit Hochrisikoprofil (Gualdi et al. 2000; Mandelson et al. 2000; Delorme 2001; Schnall 2001; Komatsu et al. 2004; Kurz et al. 2006; Fischer U 2010). Zudem stellt die MRT die Methode der Wahl bei der Untersuchung von Patienten mit Brustimplantaten dar (Holmich et al. 2005).

\subsection{Terminologie und Auswertekriterien in der Mamma-MRT}

In dem vom ACR entworfenen BIRADS-MRI-LEXICON werden auffällige Befunde in der MRT als Fokus (focus), Herdbefund (mass) oder als nicht-raumfordernde Anreicherung (non-mass-like enhancement) unterschieden (American College of Radiology 2004).

Als Fokus bezeichnet man eine fleckförmige Anreicherung $<5 \mathrm{~mm}$, die aufgrund ihrer geringen Größe morphologisch nicht sicher beschrieben werden kann. Foki treten in der Regel bilateral multipel auf und sind in erster Linie Ausdruck physiologischer Durchblutungsphänomene.

Ein Herdbefund ist eine dreidimensionale Läsion, für deren Beschreibung die morphologischen Auswertkriterien der Form, Begrenzung und des Binnenmusters angewandt werden. Die Form beschreibt das Erscheinungsbild einer mit Kontrastmittel angereicherten Region. Unterschieden werden rund, oval, lobuliert und irregulär. Die Begrenzung stellt die Kontur einer kontrastmittelanreichernden Region dar. Hier werden glatt, irregulär und spikuliert differenziert. Ovale, runde und lobulierte Form sowie glatte Begrenzung weisen eher auf benigne Tumoren hin, während irreguläre Konturen und Formen häufig auf Malignität hindeuten. Das Binnenmuster gibt Auskunft über die endotumorale Kontrastmittelverteilung. Die Verteilung kann homogen, inhomogen oder ringförmig (sog. rim enhancement) sein. Insbesondere das Ringenhancement ist häufig 
mit Malignität assoziiert, da sich die tumorbiologisch aktive Zone bei Karzinomen typischerweise im Randbereich befindet (Buadu et al. 1997).

Kann eine pathologische Anreicherung weder einem Fokus noch einem Herdbefund zugeordnet werden, handelt es sich um eine nicht raumfordernde Anreicherung. Diese respektiert typischerweise das umgebene intramammäre Fettgewebe. Solche "nonmass-like" Läsionen werden, nach den Kriterien der Anreicherungsform (fokal, linear, duktal, segmental, regional, diffus), des Anreicherungsmusters (homogen, heterogen, punktförmig, gruppiert, dendritisch) und dem Aspekt der Symmetrie eingeteilt und ausgewertet.

\subsection{Kontrastmitteldynamik in der Mamma-MRT}

Neben den morphologischen Kriterien werden insbesondere Herdbefunde in der MRT hinsichtlich der Aufnahme und Abgabe des Kontrastmittels evaluiert. Diese Kontrastmitteldynamik wird beschrieben als zeitliche Veränderung der Signalintensität von kontrastmittelaufnehmenden Strukturen nach Applikation einer paramagnetischen Substanz. Erfasst wird die Dynamik in geeigneten Messregionen (sog. ROI, region of interest). Die resultierende Signalintensitäts-Zeit-Kurve wird in eine Initialphase (1-3 Minute nach Kontrastmittelinjektion) und in eine Postinitialphase (3-8 Minuten nach Kontrastmittelinjektion) unterteilt (Fischer $U$ et al. 1993). Die Geschwindigkeit des Signalintensitätsanstiegs zum Ausgangswert der Nativuntersuchung wird in gering $(<50 \%)$, mäßig (50-100\%) und stark (>100\%) eingeteilt (Abb. 4). Ein persistierender Signalanstieg in der Postinitialphase wird als ein kontinuierlicher Anstieg, ein horizontaler Kurvenverlauf als Plateau und eine abfallende Kurve als wash out beschrieben (Abb. 5). 


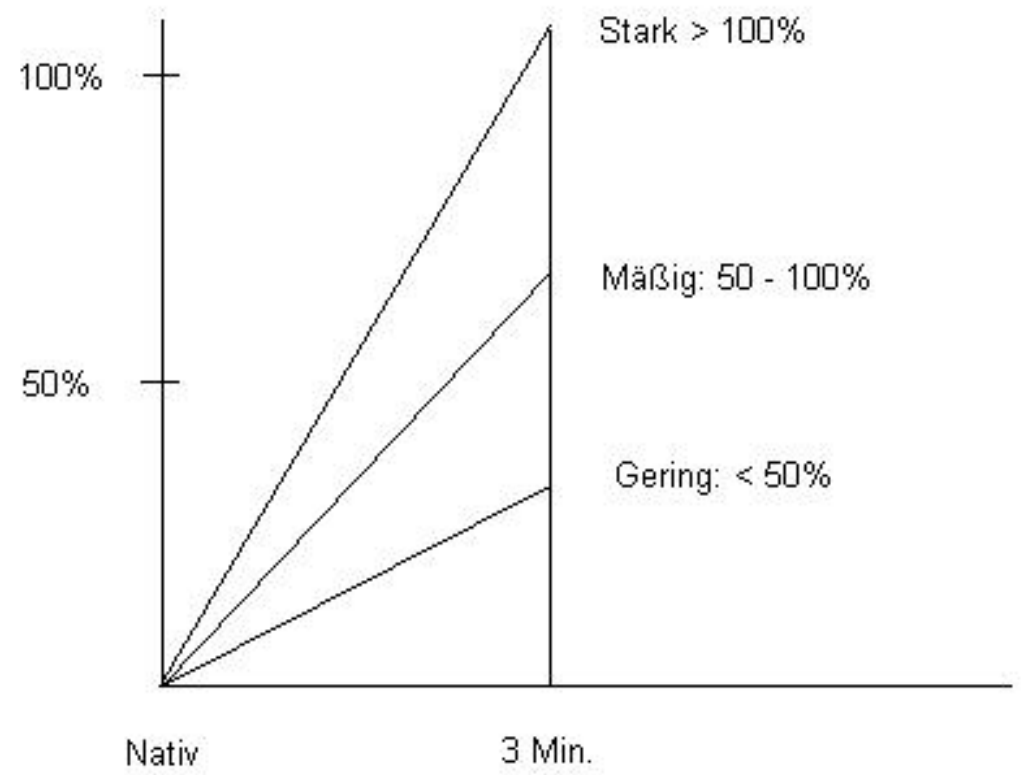

Abbildung 4: Initialer Signalanstieg. Abbildung aus Fischer U (2000), Seite 28.

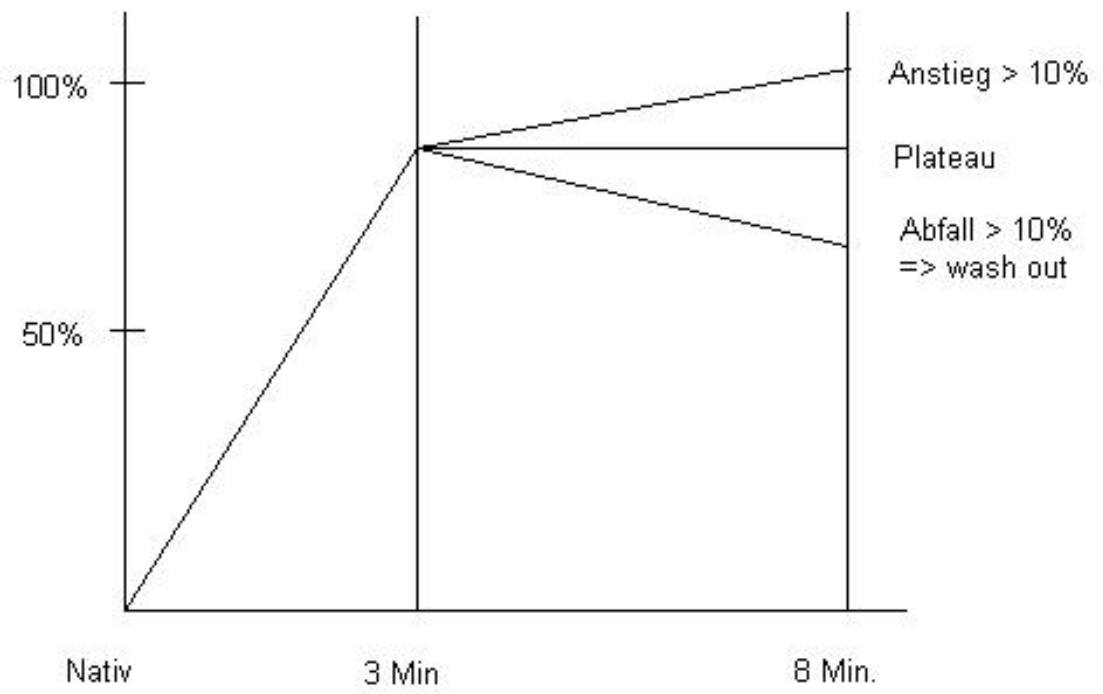

Abbildung 5: Postinitialer Signalverlauf. Abbildung aus Fischer U (2000), Seite 29.

Die meisten Karzinome weisen ihren maximalen Signalanstieg innerhalb der ersten 3 Minuten nach Kontrastmittelgabe auf, dem ein Plateau oder häufig ein Auswaschphänomen in dem postinitialen Signalverlauf folgt. Pathophysiologisches 
Korrelat des frühzeitigen Auswaschphänomens sind die Tumorangiogenese mit der verstärkten Gefäßdichte, die erhöhe Gefäßpermeabilität der ausgebildeten Gefäße sowie tumorassoziierte Shunts, die ein rasches Auswaschen begünstigen. Benigne Veränderungen zeigen im Gegensatz hierzu eher einen mehr oder weniger langsamen, fast linearen Signalanstieg auf (Orel et al. 1994; Siegmann et al. 2002; Fischer U 2010).

\subsection{Der Göttingen-Score}

Das BIRADS MRI-LEXICON des ACR präsentiert zwar zum einen eine einheitliche Befundbeschreibung für die Mamma-MRT und zum anderen eine schlüssige Kategorisierung der erhobenen Befunde. Es führt jedoch nicht aus, welche Befundkonstellation zu welcher Einstufung innerhalb der MRM-BIRADS-Kategorisierung führt. Um die Dignität einer Läsion abschätzen zu können, erarbeiteten Fischer $U$ et al. daher ein Score-System, das den morphologischen und dynamischen Kriterien in Abhängigkeit vom konkreten Befund einen Punktwert zuordnet. Befunde, die häufig mit Malignität assoziiert sind, werden hierbei höher gescort als solche Veränderungen, die eher mit Gutartigkeit einhergehen. Starker initialer Anstieg, wash out und rim enhancement erhalten hierbei eine maximal zu vergebene Punktzahl von je 2. Die Summation aller Einzelkriterien führt schlussendlich zu einer Gesamtpunktzahl eines Befundes von 0-8 (Tab. 2). Befunde mit einer Punktzahl $<3$ weisen hierbei auf eine benigne Läsion, solche mit einer Gesamtpunktzahl >3 eher auf eine bösartige Veränderung hin. Überträgt man den Göttingen-Score in die BIRADS- Klassifikation, so ergibt sich für Scorewerte 0 bis 2 ein Befund der Kategorie MRM-BIRADS 1 und MRMBIRADS 2. Eine Gesamtpunktzahl von 3 ist einer MRM-BIRADS Kategorie 3 zuzuordnen. Scorewerte von 4 und 5 Punkten fallen in die Kategorie MRM-BIRADS 4. Höhere Werte entsprechen der Rubrik MRM-BIRADS 5 (Fischer U et al. 1993; Fischer U 2010). 


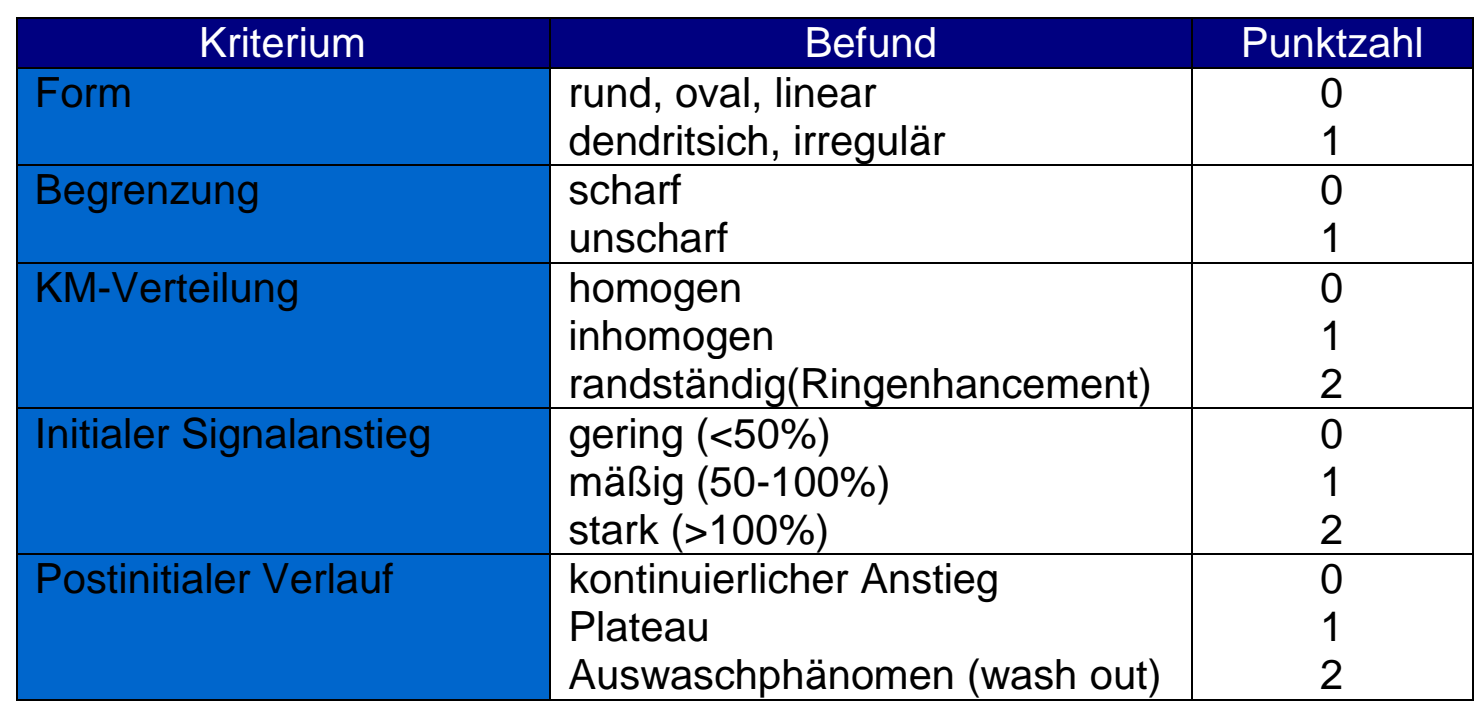

Tabelle 2: Göttingen-Score nach Fischer U. Nach Fischer U (2000) Seite 30.

In Analogie zur Beurteilung des Mammogramms und der Sonographie wurde durch das American College of Radiology im Jahre 2004 auch eine einheitliche Terminologie und „BIRADS-Klassifizierung“ für die Mamma-MRT (BIRADS 0-6) inauguriert (American College of Radiology 2004)

\section{MRM-BIRADS Kategorie 0:}

MRT ist nicht abschließend zu beurteilen. Weitere Bildgebung (z.B. Mammographie, Sonographie) ist erforderlich.

\section{MRM-BIRADS Kategorie 1:}

MRT ist abschließend zu beurteilen. Es liegt kein relevanter Befund im Mammogramm vor. Es finden sich keine Herdbefunde oder architektonischen Störungen. Karzinomrisiko $0 \%$.

\section{MRM-BIRADS Kategorie 2:}

MRT ist abschließend zu beurteilen. Die Mamma-MRT zeigt einen beschreibenswerten Befund auf, der aufgrund von Form, Begrenzung und Vaskularisation eindeutig als benigne einzustufen ist (z.B. Zyste, Ölzyste, Lymphknoten). Karzinomrisiko 0\%. 


\section{MRM-BIRADS Kategorie 3:}

MRT ist abschließend zu beurteilen. Es zeigt sich eine Auffälligkeit im MRT, die mit hoher Wahrscheinlichkeit benigne ist. Karzinomrisiko <2\%. Es wird eine kurzfristige Verlaufskontrolle, üblicherweise nach 6 Monaten, empfohlen.

\section{MRM-BIRADS Kategorie 4:}

MRT ist abschließend zu beurteilen. Es findet sich im MR-Mammogramm ein suspekter Befund, der jedoch nicht die typischen Charakteristika eines Malignoms aufweist. Karzinomrisiko 2-90\%. Eine Unterteilung in die Kategorien BIRADS 4a (Risiko 2-30\%), BIRADS 4b (30-60\%) und BIRADS 4c (60-90\%) ist möglich. Eine histologische Abklärung des Befundes, bevorzugt durch eine perkutane Biopsie, wird empfohlen.

\section{MRM-BIRADS Kategorie 5:}

MRT ist abschließend zu beurteilen. Es findet sich ein Befund, der die typischen Kriterien eines Karzinoms aufweist. Karzinomrisiko $>90 \%$. Der Befund stellt eine Indikation für eine histologische Abklärung dar, damit anschließend adäquate therapeutische Maßnahmen eingeleitet werden können.

\section{BIRADS Kategorie 6:}

Die Malignität einer Auffälligkeit in der MRT wurde histologisch nach einer perkutanen Biopsie gesichert. Geeignete onkologische Maßnahmen werden erforderlich. 


\section{Material und Methoden}

\subsection{Patientenauswahl und Studienpopulation}

Im Rahmen dieser Studie wurden alle diagnostischen Mamma-MRT-Untersuchungen, die im Diagnostischen Brustzentrum Göttingen innerhalb eines 6-jährigen Zeitraumes (2003 bis 2009) durchgeführt worden waren, erfasst. Es wurden solche MRTUntersuchungen vermerkt und alphabetisch nach Nachnamen aufgelistet, die Anhand des zu jeder Untersuchung vorliegenden schriftlichen Befundes als MRM-BIRADS 3 oder MRM-BIRADS 4 kategorisiert worden waren. Aus jeder Gruppe wurden etwa 200 Fälle konsekutiv in die Evaluation dieser Studie integriert. Die Auswahl dieser Untersuchungen erfolgte somit unabhängig von der Indikation, die zur Durchführung der MRT Veranlassung gab. Die Befunde der üblicherweise ebenfalls angefertigten Röntgenmammographie bzw. Mammasonographie gingen ebenfalls nicht in die Evaluation der Daten ein.

\subsection{Durchführung des diagnostischen Mamma-MRT}

Die Mamma-MRT-Untersuchungen waren ausnahmslos in Bauchlage an einem 1.5T Ganzkörpermagneten der Fa. GE Health Care (Milwaukee, WI, USA) unter Verwendung einer dedizierten offenen 4-Kanal-Mamma-Oberflächenspule (MRI Device, Fa. Invivo, Gainesville, FL, USA) mit integriertem Kompressorium „CC-Fixiereinheit“ der Fa. Noras (Fa. Noras MRI Products $\mathrm{GmbH}$, Hoechberg) angefertigt worden. Folgendes Protokoll kam für alle MRT-Untersuchungen zur Anwendung:

Primär erfolgte eine wassersensitive Messung mit einer T2-Inversion-Recovery-Sequenz in 2D-Technik (TR 6925ms, TE 67ms, Inversionszeit 150ms, Matrix $512 \times 224$, FOV 38 $\mathrm{cm}$, Einzelschichtdicke 2,5mm, Schichtpositionierung identisch zur nachfolgenden T1Messung, Angulierung axial, Messzeit 4:51 min). Anschließend wurde eine dynamische kontrastmittelsensitive T1-Gradienten-Echo-Sequenz „Vibrant“ in 3D-Technik angefertigt (TR 8,4ms, TE 4,1ms, Matrix $512 \times 512$, FOV $38 \mathrm{~cm}$, Schichtdicke 2,5mm, Angulierung axial, Messzeit 1:26min repetitiv einmalig vor und 5-malig nach peripher-venöser Gabe 
von $0,1 \mathrm{mmol}$ Gd-DTPA/kg Körpergewicht [Magnevist $\AA$, Fa. Schering, Berlin, später Bayer Healtcare, Leverkusen]).

Das Postprocessing der dynamischen kontrastmittelverstärkten Aufnahmen umfasste eine obligate Bildsubtraktion der 2. Messung nach KM-Gabe abzüglich der Nativbilder (Frühsubtraktion), fakultativ auch eine Subtraktion der 1. Messung nach KM-Gabe abzüglich der Nativaufnahmen (Frühstsubtraktion). Die Signalanalyse erfolgte im Maximum der Anreicherung einer als suspekt erachteten Läsion mit einer an den jeweiligen Befund angemessenen Region-of-Interest (ROI) von 2-5 Pixeln.

In allen Fällen erfolgte eine Befundverschlüsselung der abzuklärenden Läsionen durch den jeweils untersuchenden Facharzt in Abhängigkeit vom magnetresonanztomografischen Erscheinungsbild: In Anlehnung an die ACRTerminologie wurde unterschieden zwischen Fokus/Foci (Läsionen $<5 \mathrm{~mm}$ Durchmesser), Herdbefunden (raumfordernde Veränderungen $\geq 5 \mathrm{~mm}$ ) und nicht raumfordernden Läsionen (sog. Non-mass-like lesions, NML). Herdbefunde wurden zusätzlich nach dem sog. Göttingen-Score, bei dem für verschiedene morphologische und dynamische Kriterien in Abhängigkeit vom Befund Punkte vergeben werden, evaluiert. Aus der Gesamtpunktzahl wurde eine MRM-BIRADS-Kategorisierung abgeleitet und im schriftlichen Befund vermerkt.

Die Indikation zur MR-gesteuerten Vakuumbiopsie wurde ausschließlich in solchen Fällen gestellt, in denen es weder mammografisch noch sonografisch ein zuverlässiges bildgebendes Korrelat zum auffälligen Befund in der MRT gab. In diesem Zusammenhang wurde bei allen 221 Patientinnen, die in der eigenen Abteilung vordiagnostiziert wurden, vor der geplanten MR-gesteuerten Vakuumbiopsie eine Second-Look-Sonografie in Kenntnis der genauen Tumorgröße und -lage durchgeführt. Im präsentierten Kollektiv wurde auf die Second-Look-Sonografie bei Patientinnen, die von externen Untersuchern zugewiesen wurden, aus mehreren Gründen verzichtet: Prinzipiell wurden diese Patienten bereits auswärts durch die zuweisenden Kolleginnen und Kollegen einer gezielten Zweitsonografie unterzogen, sodass die 
Ultraschalluntersuchung nicht ein drittes $\mathrm{Mal}$ in der eigenen Abteilung durchgeführt wurde.

\subsection{Durchführung der MR-gestreuten Vakuumbiopsien}

Befunde der Kategorie BIRADS 4 wurden gemäß nationaler S3-Leitlinie durch eine MRgesteuerte Vakuumbiopsie perkutan-bioptisch abgeklärt. Diese Intervention erfolgte an dem o.g. 1,5T-System unter zusätzlicher Verwendung einer spulenintegrierten Punktionsvorrichtung (MRI Device, Fa. Invivo, Gainesville, FL, USA) und in definierten Einzelfällen mit weit medialem Sitz der abzuklärenden Läsion unter Verwendung einer offenen 8-Kanal-Spule mit medialem Zugangsweg (Fa. GE Health Care, Milwaukee, IL, USA). Für die MR-Vakuumbiopsie kam als Zielvorrichtung zum einen eine sog. Postand-Pillar-Einheit zum Einsatz. Dieses System erlaubt eine stufenlose Verschiebung der Nadelhalterung in $x$ - und y-Achse. Zum anderen erfolgten die Interventionen - je nach Wahl des Untersuchers - mit einem würfelartigen Gridsystem, in das perforierte Würfel integriert werden können. Nach adäquater Positionierung der Patientinnen in Bauchlage und Fixierung der abzuklärenden Brust erfolgte eine dynamische T1-gew. GE-Messung (Vibrant in 3D-Technik: TE 4,1 ms, TR $8,4 \mathrm{~ms}$, Matrix $512 \times 512$, FOV $38 \mathrm{~cm}$, Schichtdicke 2,5mm, Angulierung axial, Messzeit 1:26min repetitiv einmalig vor und 3malig nach i.v. Gabe von 0,1 mmol Gd-DTPA/kg Körpergewicht). Anschließend wurde eine Frühsubtraktion zur Reproduzierbarkeit des abzuklärenden Befunds durchgeführt. Es folgte eine Berechnung geeigneter Punktions-Koordinaten für die $x-, y-$ und die zAchse. Die Gewebeentnahme erfolgte entweder mit dem 9 G-System Vacora (Fa. Medicor, Kempten, Deutschland) oder mit dem 10 G-System ATEC (Automated Tissue Excision and Collection, Fa. Suros Surgical Systems, Indianapolis, IN, USA). Die Vorgehensweise der MR-gesteuerten Vakuumbiopsie erfolgte in Anlehnung an die Empfehlungen der AG Mammadiagnostik der Deutschen Röntgengesellschaft für MRgesteuerte Interventionen (Bick et al. 2008). 


\subsection{Erhebung der Daten im Rahmen dieser Studie}

Alle im Rahmen dieser Studie ausgewerteten Mamma-MRT-Untersuchungen, die ausnahmslos als DICOM-Datensatz zur Verfügung standen, wurden nach einem standardisierten Protokoll und in Unkenntnis der primär erhobenen Befunde im Konsensus zwischen einem unerfahrenen Auswerter (F.R.) und einem seit 27 Jahren in der Mamma-MRT erfahrenen Radiologen (U.F.) erneut ausgewertet. Hierbei wurden folgende Kriterien evaluiert:

Zuordnung der jeweiligen Befunde zur Befundgruppe Fokus $(<5 \mathrm{~mm})$, Herd (raumfordernde Läsion $\geq 5 \mathrm{~mm}$ ) oder NML (nicht-raumfordernde Läsion).

In der Befundgruppe „Fokus“ wurden die Läsionsgröße $(1 \mathrm{~mm}, 2 \mathrm{~mm}, 3 \mathrm{~mm}, 4 \mathrm{~mm})$ und der Wassergehalt in der wassersensitiven IR-Messung (hypo-, iso-, hyperintens) evaluiert.

In der Befundgruppe ${ }_{\Perp}$ Herd“ wurden ebenfalls die Läsionsgröße $(5-10 \mathrm{~mm}, 10-15 \mathrm{~mm}, 15-$ $20 \mathrm{~mm}$ ), die Form des Herdbefundes (rund, oval, lobuliert, irregulär), die Begrenzung (glatt, unscharf), die endotumorale Verteilung des Kontrastmittels im Herd (homogen, inhomogen, rim), der initiale Signalanstieg im Maximum des Enhancements (gering: $<50 \%$, mäßig: 50-100, stark: $>100 \%$ ), das postinitiale Signalverhalten (kontinuierlicher Anstieg, Plateau, wash out), der Wassergehalt in der wassersensitiven IR-Messung (hypo-, iso-, hyperintens) sowie der Nachweis endotumoraler Septierungen (dunkle Septen, helle Septen, zentrales Enhancement) ausgewertet. Aus den o.g. Kriterien wurde zusätzlich gemäß Göttingen-Score eine Gesamtpunktzahl pro Herdbefund errechnet.

In der Befundgruppe „NML“ erfolgte eine Auswertung der Ausdehnung der Läsion (0$5 \mathrm{~mm}, 5-10 \mathrm{~mm}, 10-20 \mathrm{~mm}, 20-40 \mathrm{~mm}, 40$ und mehr) sowie die Konfiguration des anreichernden Befundes (linear, segmental, regional, diffus). 
Im Rahmen der Auswertung erfolgte im ersten Teil eine Evaluation der jeweiligen Auswertekriterien von Fokus, Herd und NML in Abhängigkeit von der primär durchgeführten Zuordnung zur Kategorie MRM-BIRADS 3 und MRM-BIRADS 4. Hieraus sollte ggf. abgeleitet werden, ob es ein führendes Befundmuster gab, dass jeweils zur Zuordnung in eine der beiden BIRADS-Kategorien geführt hatte.

In einem zweiten Teil erfolgte eine Auswertung der jeweiligen Kriterien von Fokus, Herd und NML in Abhängigkeit von der endgültigen Histologie bzw. Einschätzung der Dignität gemäß Verlauf. Hieraus sollte ein potentiell nachweisbares Befundmuster in der MRT für gutartige, für bösartige oder für Borderline-Läsionen abgeleitet werden. Hierfür wurden die Befunde der Kategorie MRM-BIRADS 3 und 4 gemäß Aktenlage weiter ausgewertet:

In der Gruppe der primär als MRM-BIRADS 3 verschlüsselten Befunde wurde geklärt, wie viele der entsprechenden Patienten zur empfohlenen Kontroll-Untersuchung nach 6 Monaten erschienen und welcher MR-Befund sich im Rahmen dieser Kontrolle ergab.

In der Gruppe der primär als MRM-BIRADS 4 verschlüsselten Befunde, bei denen im Rahmen der Primärdiagnostik ausnahmslos eine perkutan-bioptische Abklärung durch eine Vakuumbiopsie angeraten worden war, wurde evaluiert, was die histopathologische Aufarbeitung der entnommenen Gewebezylinder ergab.

Alle histologisch gesicherten Befunde wurden den Gruppen „benigne“ (histopathologische Klassifikation: B1, B2), „Borderline-Läsion“ (Befund mit unklarem biologischen Potential, histologische Klassifikation: B3) und „maligne“ (histologische Klassifikation: B5a, B5b) zugeordnet. 


\section{Ergebnisse}

\subsection{Charakterisierung der Studienpopulation}

Im Zeitraum von 01.06.2003 bis zum 31.06.2009 wurde im Diagnostischen Brustzentrum Göttingen bei insgesamt 5182 Frauen eine diagnostische Mamma-MRT angefertigt. Hierunter waren 726 (14,0\%) Patientinnen, bei denen die Befunde der MRT der Kategorie MRM-BIRADS 3 oder MRM-BIRADS 4 zugeordnet wurden. 321 (6,2\%) dieser Befunde wurden der Kategorie BIRADS 3 zugewiesen, 405 (7,8\%) der Kategorie BIRADS 4. In alphabetischer Reihenfolge wurden innerhalb dieser Studie 196 Patienten mit einem Befund der Kategorie MRM-BIRADS 3 und 198 Patienten mit einem Befund der Kategorie MRM-BIRADS 4 ausgewertet. Das Gesamtkollektiv betrug somit 394 Befunde.

Das Alter der Patientinnen mit einem Befund nach MRM-BIRADS 3 lag zum Zeitpunkt der Mamma-MRT zwischen 27 und 71 Jahren (Durchschnittsalter: 48,9 Jahre). Das Alter der Patientinnen mit einem Befund der Kategorie BIRADS 4 lag zum Zeitpunkt der MRT zwischen 23 und 79 Jahren (Durchschnittsalter: 51,6 Jahre).

Herd (46\%) und nicht-raumfordernde Läsion (43\%) stellten im Gesamtkollektiv die häufigsten Befunde dar, während nur etwa 10\% der auffälligen Veränderungen einem Fokus entsprachen (Abb. 6). 


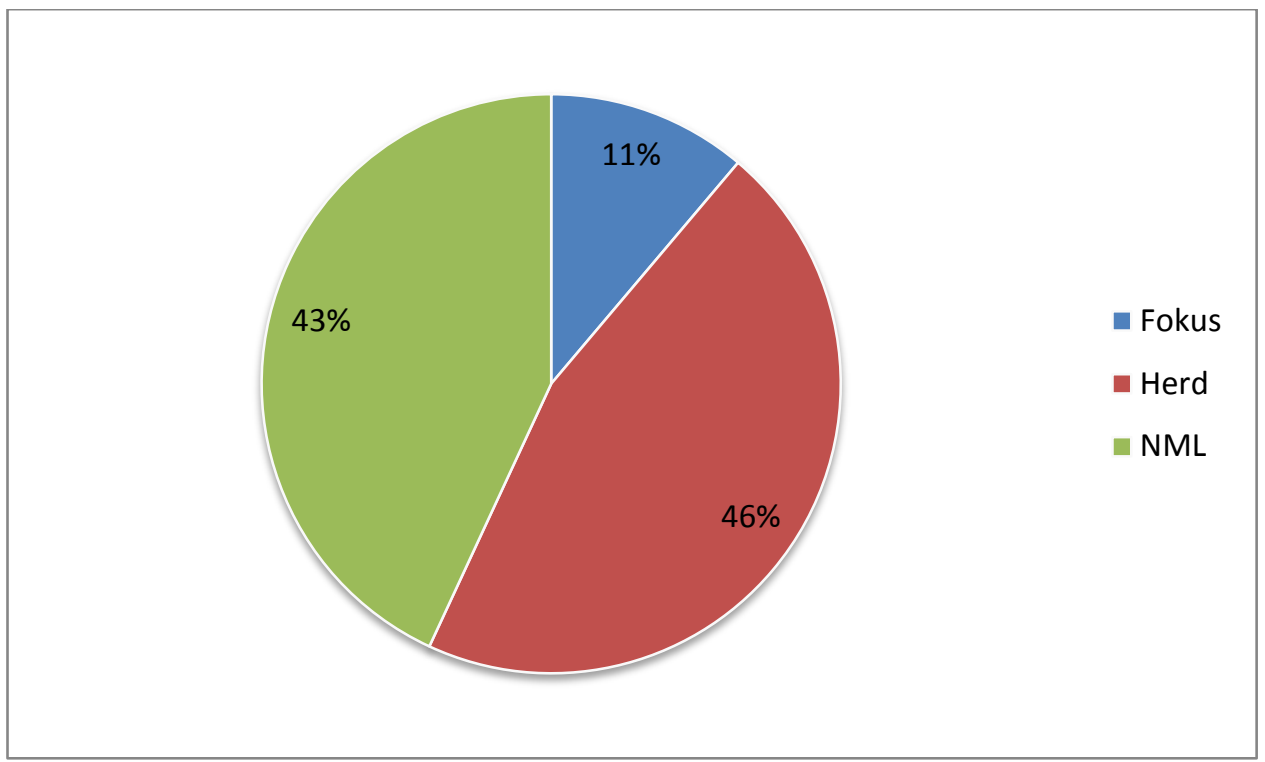

Abbildung 6: Prozentuale Verteilung der 3 Formen anreichernder Läsionen im gesamten Patientenkollektiv.

Im Vergleich der beiden Befundkategorien MRM-BIRADS 3 und MRM-BIRADS 4 zeigte sich, dass ein milder Übergang der Herde und nicht-raumfordernden Läsionen in der Kategorie BIRADS 4 vorlag, während bei den Foki die Kategorie MRM-BIRADS 3 geringfügig häufiger vorkam (Abb. 7).

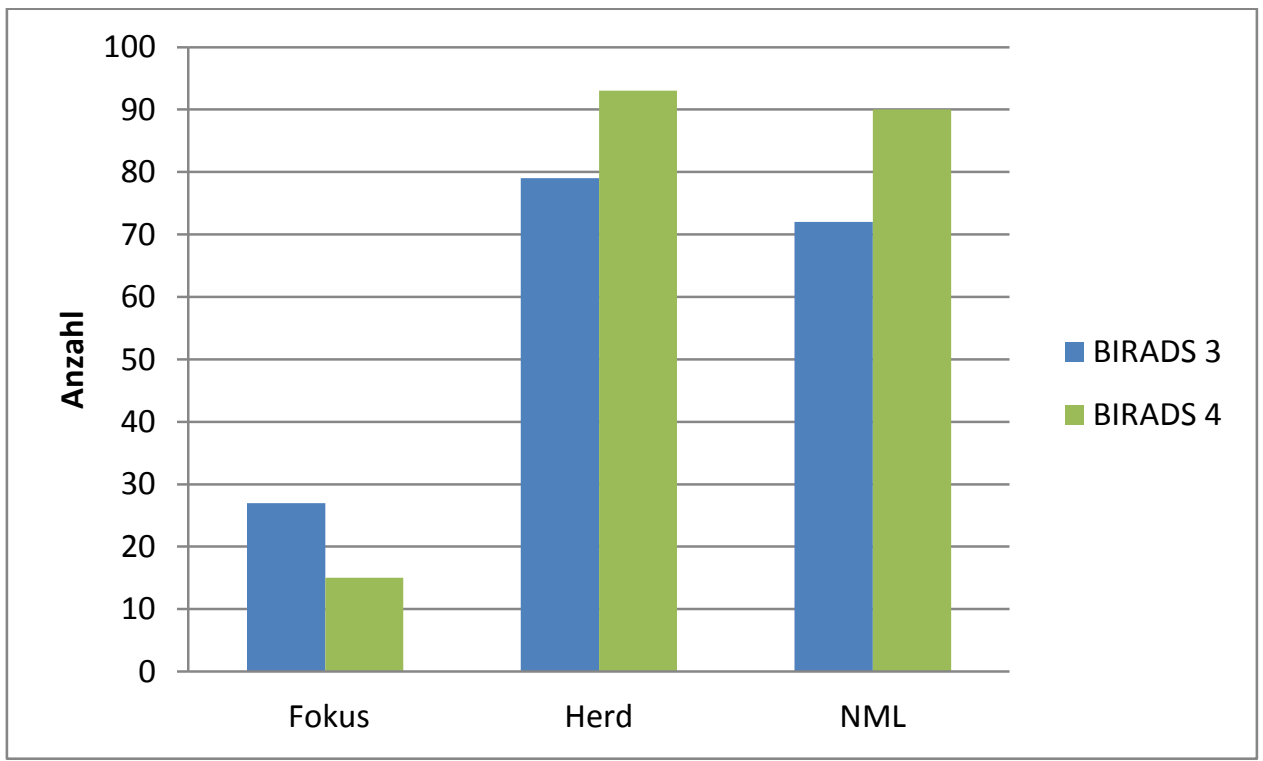

Abbildung 7: Anzahl der Foki, Herdbefunde und NML in Abhängigkeit von der MRM-BIRADS-Kategorie. 


\subsection{Spezielle Auswertung}

3.2.1 MR-tomografische Läsionsbeurteilung der Foki

Von den insgesamt 27 Foki, die der Kategorie MRM-BIRADS 3 zugewiesen wurden, wiesen mehr als die Hälfte eine Größe von $4 \mathrm{~mm}$ auf, etwa $40 \%$ waren $3 \mathrm{~mm}$ und weniger als $5 \%$ noch kleiner. Die Foki in der Kategorie MRM-BIRADS 4 stellen sich durchschnittlich größer dar, wobei 80\% eine Größe von 4mm aufwiesen (Abb. 8).

Bei knapp einem Sechstel (14,8\%) der Kategorie 3 stellte sich eine endotumorale Septierung innerhalb eines Fokus (Hamburgerzeichen) dar. In der Kategorie 4 lag diese Quote bei 7,1\%, allerdings bei einer geringen Gesamtanzahl von 14 Foki.

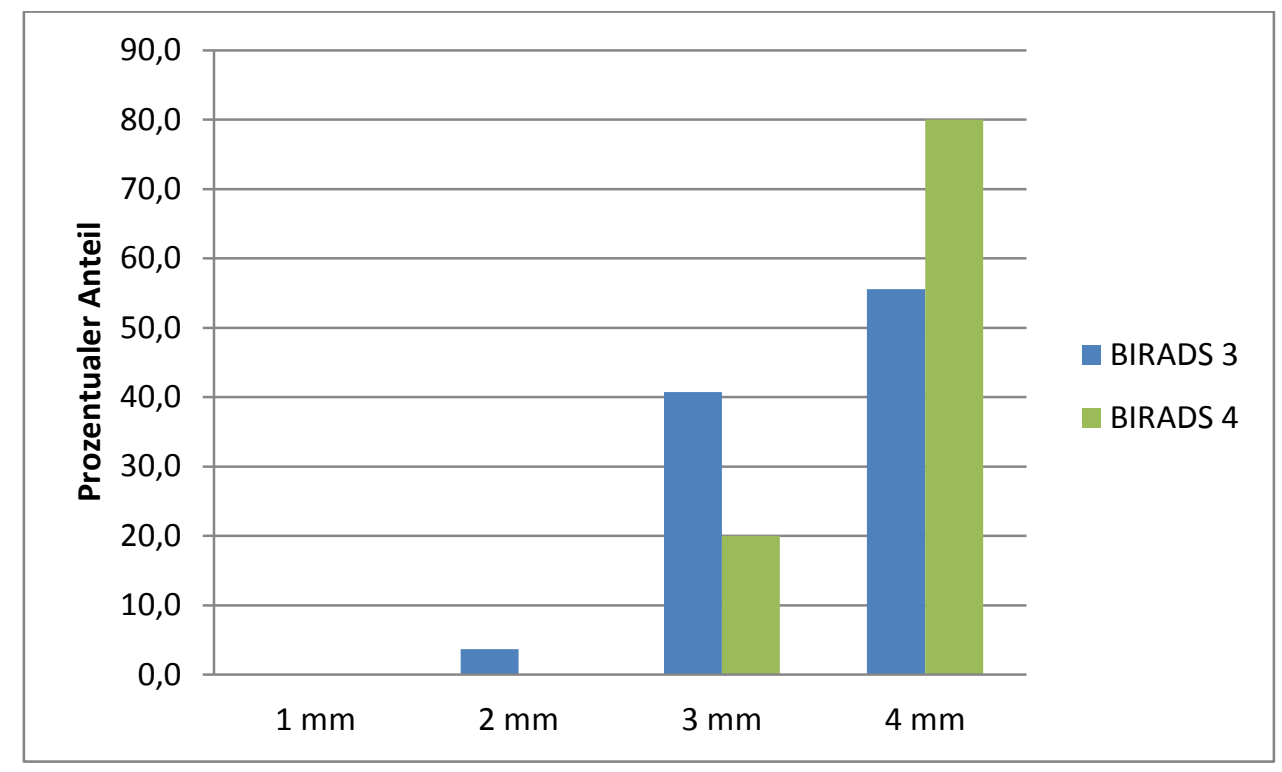

Abbildung 8: Fokusgröße in Abhängigkeit von der MRM-BIRADS-Kategorie.

Abbildung 9 veranschaulicht den endotumoralen Wassergehalt der Foki im Bezug auf das umliegende Drüsenparenchym. Hier ist zu erkennen, dass der Wassergehalt der Tumoren der Kategorie BIRADS 3 in über der Hälfte der Foki gegenüber dem angrenzenden Mammagewebe erhöht war. Im Gegensatz hierzu ist der endotumorale Flüssigkeitsgehalt bei den Foki der Kategorie MRM-BIRADS 4 in $80 \%$ der Fälle intermediär oder erniedrigt gewesen (Abb. 9). 


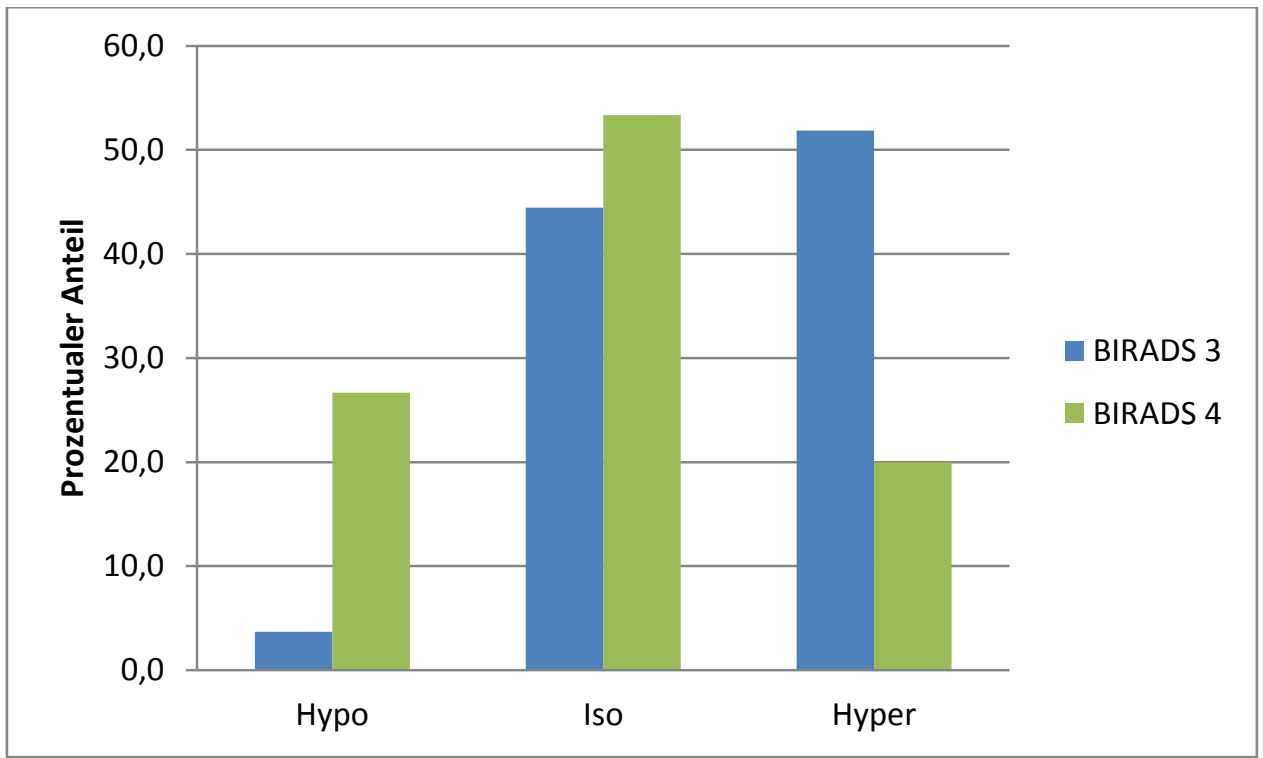

Abbildung 9: Endotumoraler Wassergehalt der Foki in Abhängigkeit zur MRM-BIRADS-Kategorisierung.

\subsubsection{MR-tomografische Läsionsbeurteilung der Herde}

Abbildung 10 gibt die Größe der Herdläsionen wieder. Hier ist zu erkennen, dass sich der wesentliche Anteil der Tumoren sowohl bei den Läsionen der Kategorie MRMBIRADS 3 (88,6\%), als auch bei denen der Kategorie MRM-BIRADS 4 (77,4\%) in der Größenordnung zwischen 5 und $10 \mathrm{~mm}$ befand. Tumoren über $10 \mathrm{~mm}$ Durchmesser stellten sich in beiden Kategorien in jeweils nur etwa $20 \%$ dar (Abb. 10). 


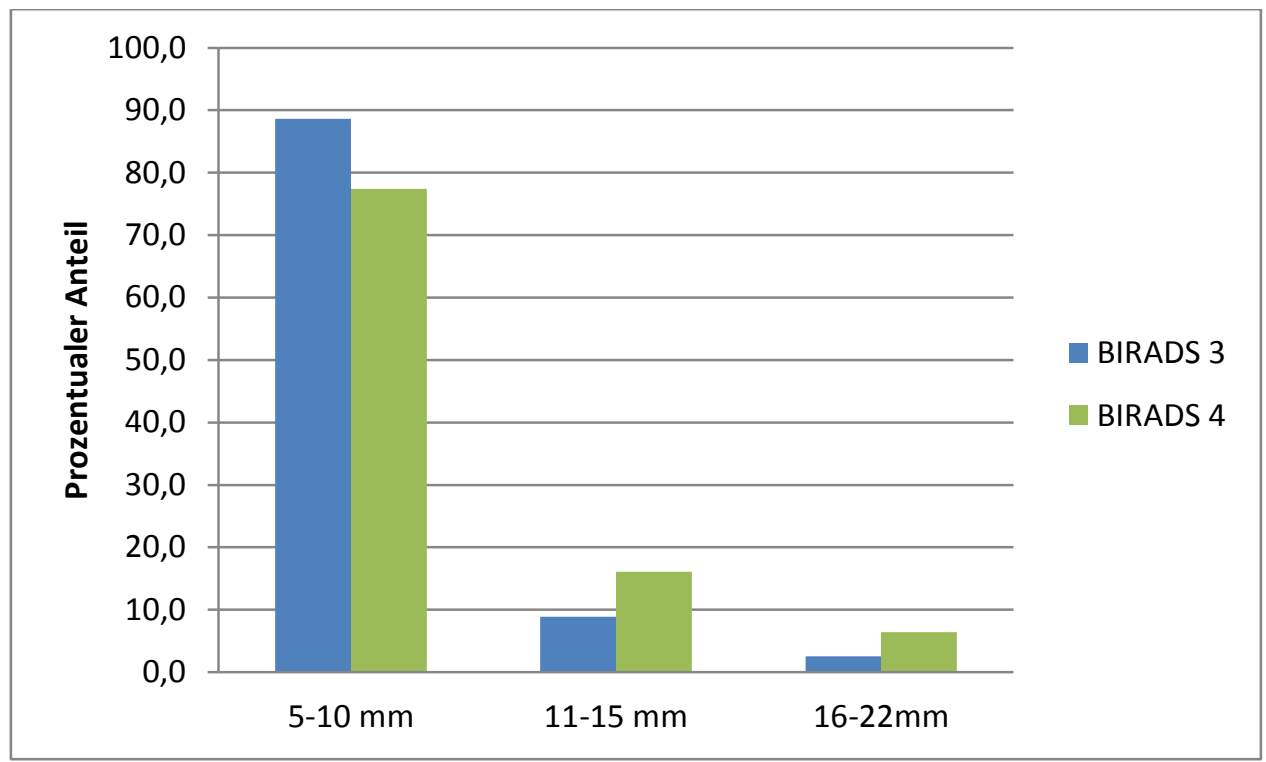

Abbildung 10: Größe des Herdbefundes in Abhängigkeit von der MRM-BIRADS-Kategorie.

Betrachtet man die Form der kontrastmittelanreichernden Herde, so ist zu erkennen, dass sich für die Kategorie MRM-BIRADS 3 überwiegend Formen abzeichneten, die mit Gutartigkeit assoziiert sind (rund, oval, lobuliert), während irreguläre Formen nur in 10\% der Fälle vorlagen. Dieses Phänomen fand sich auch für die Kategorie MRM-BIRADS 4, wenngleich die Quote der Herde mit irregulärer Form hier mit 18\% etwas höher lag (Abb. 11).

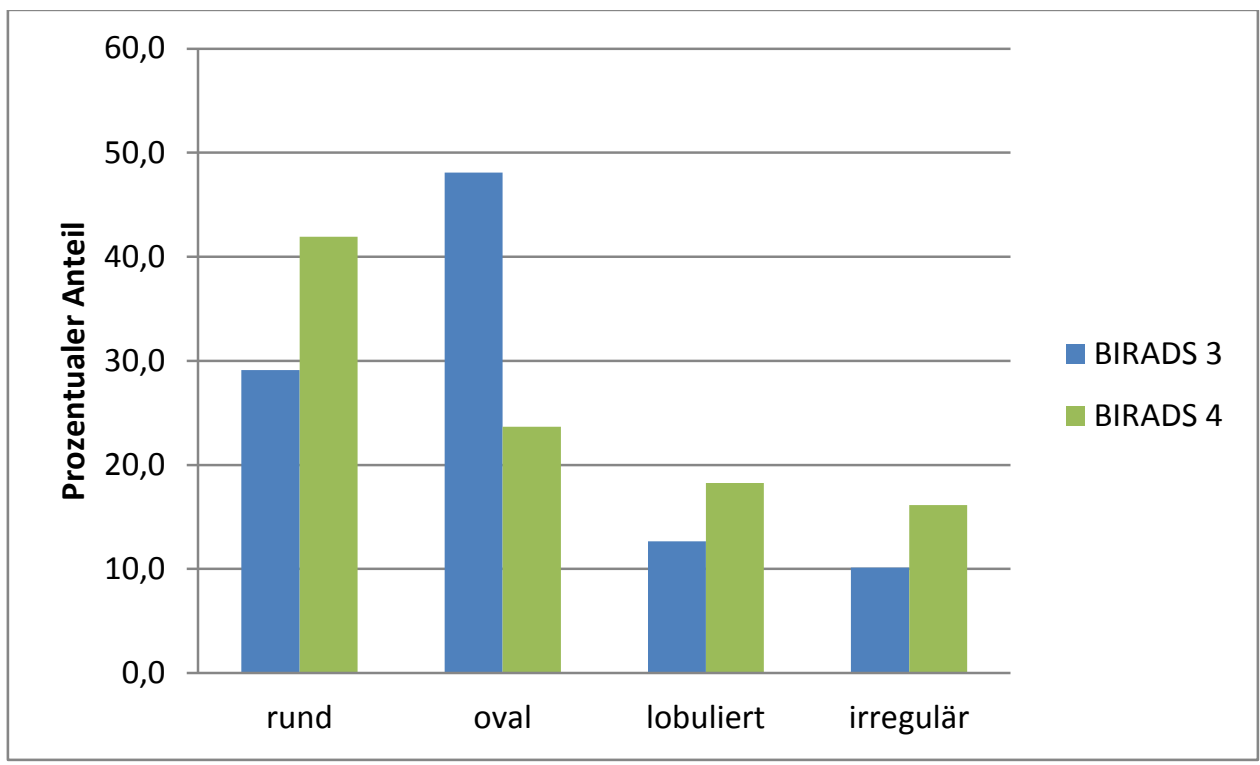

Abbildung 11: Form der Herdbefunde in Abhängigkeit von der MRM-BIRADS-Kategorie. 
Bei der Auswertung der Begrenzung der Herdbefunde war zu erkennen, dass sich die glatte Kontur in der MRM-BIRADS 3 Kategorie fast doppelt so oft darstellte wie in der MR-BIRADS 4 Gruppe. Im Gegensatz hierzu lag die unscharfe Begrenzung in der Kategorie 4 mit circa $70 \%$ nahezu doppelt so häufig vor wie in der Kategorie MRMBIRADS 3 (Abb. 12).

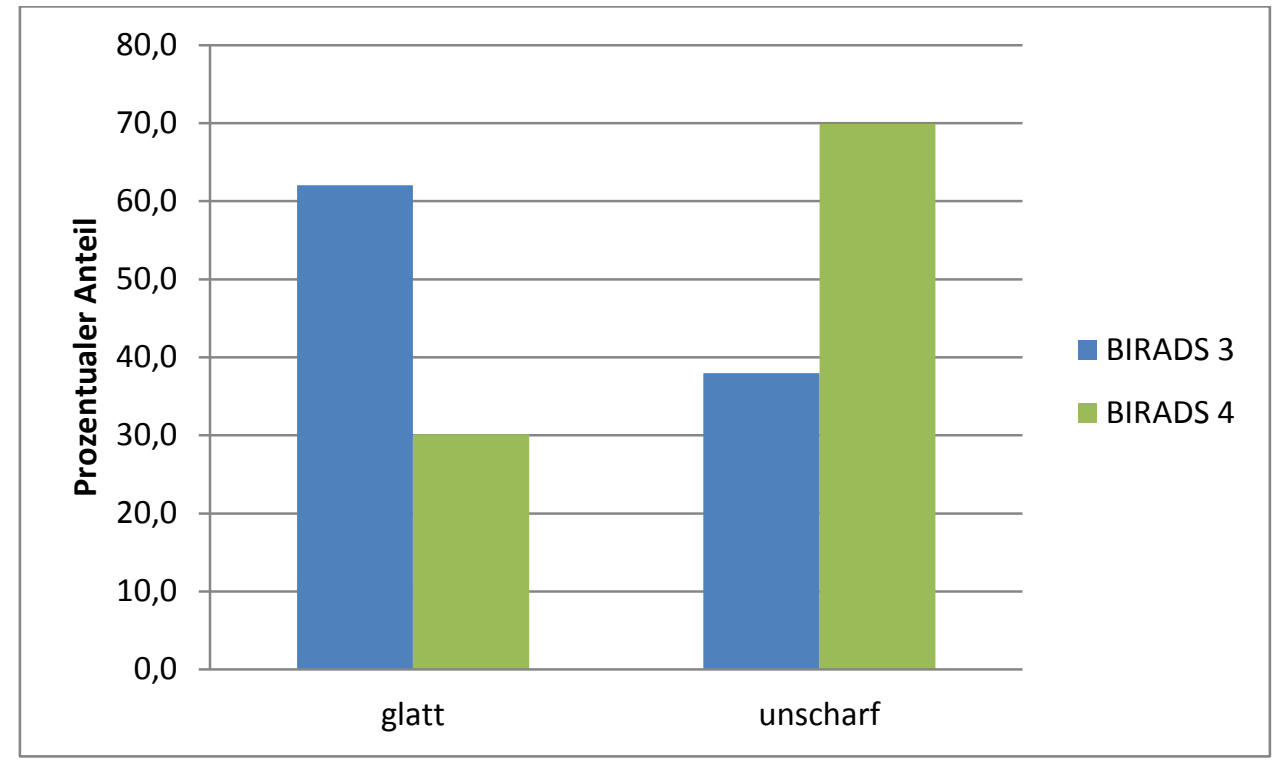

Abbildung 12: Begrenzung der Herde in Abhängigkeit von der MRM-BIRADS.

Hinsichtlich der endotumoralen Anreicherung des Kontrastmittels zeigten MRM-BIRADS 3 Befunde in $90 \%$ ein homogenes oder inhomogenes und nur in $10 \%$ der Fälle ein primär randständiges Enhancement (Ringenhancement). Im Gegensatz hierzu stellte sich in der Kategorie MRM-BIRADS 4 ein solches Ringenhancement in über $30 \%$ der Fälle dar. Eine homogene Anflutung des Kontrastmittels lag in dieser Gruppe jedoch nur selten (12\%) vor (Abb. 13). 


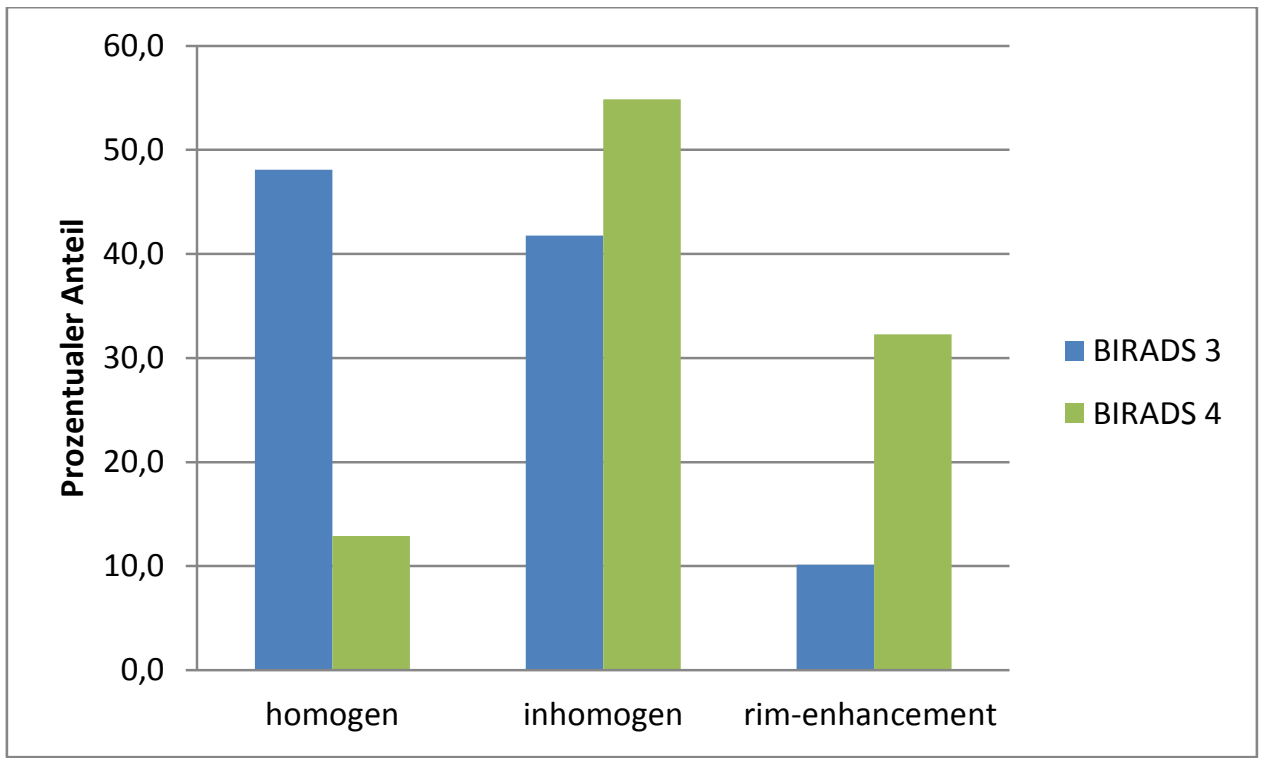

Abbildung 13: Endotumorale Kontrastmittelverteilung der Herde versus MRM-BIRADS-Kategorie.

Die Auswertung der initialen Kontrastmittelanreicherung innerhalb der ersten 3 Messminuten zeigte, dass für beide MRM-BIRADS Kategorien in sehr ähnlicher Weise ein moderates oder aber ein starkes Enhancement vorlag. Befunde mit einer initialen Steigerung des Signals um weniger als 50\% kamen in beiden Gruppen so gut wie gar nicht vor (Abb. 14).

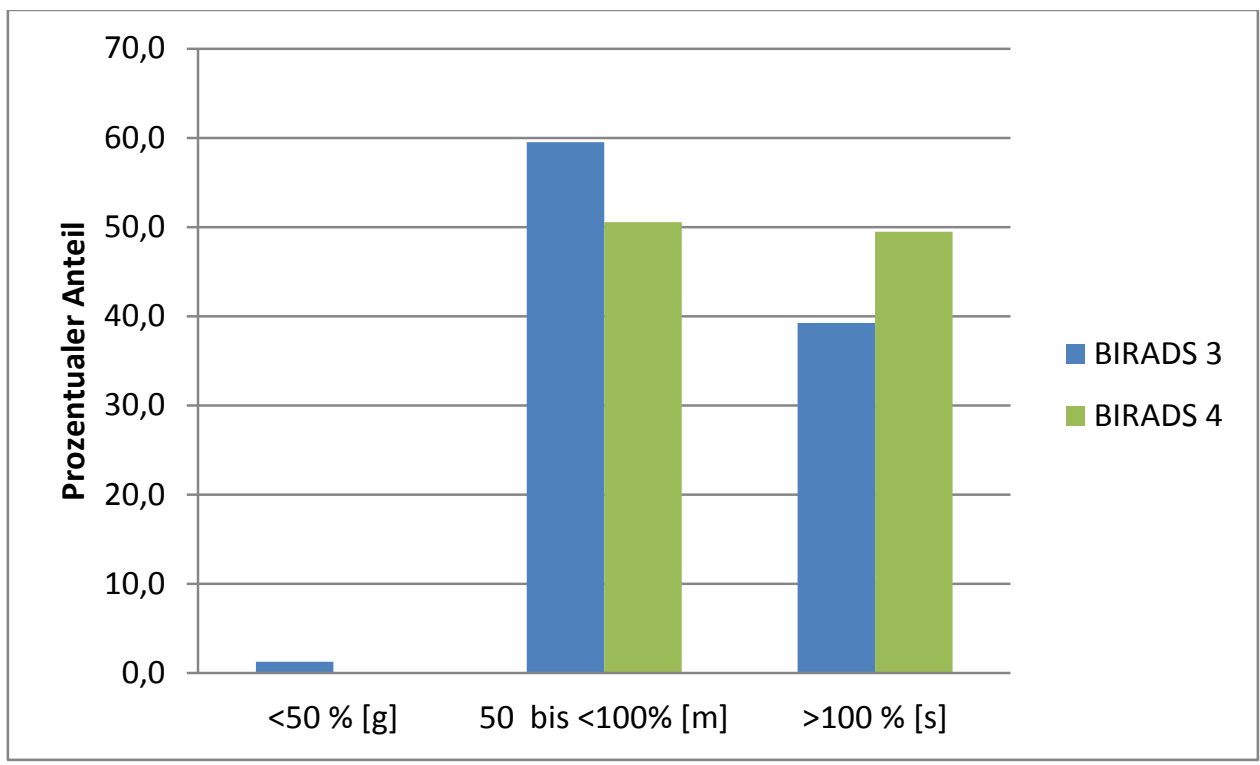

Abbildung 14: Initialer Signalanstieg der Herde in Abhängigkeit von der MRM-BIRADS-Kategorie. 
In der postinitialen Phase der Kontrastmitteldynamik zeigte sich für Befunde der Kategorie MRM-BIRADS 3 in einem Fünftel der Fälle ein kontinuierlicher Anstieg. In der MRM-BIRADS Kategorie 4 fand sich ein solcher Signalanstieg nur bei jeder 10. Patientin. In beiden Gruppen dominierte das postinitiale Plateau mit 68,4\% (MRMBIRADS 3) bzw. 54,8\% (MRM-BIRADS 4). Ein Auswaschphänomen lag in der Kategorie MRM-BIRADS 4 in einem Drittel der Fälle vor und fand sich in der Kategorie MRMBIRADS 3 in nur 12,7\% (Abb. 15).

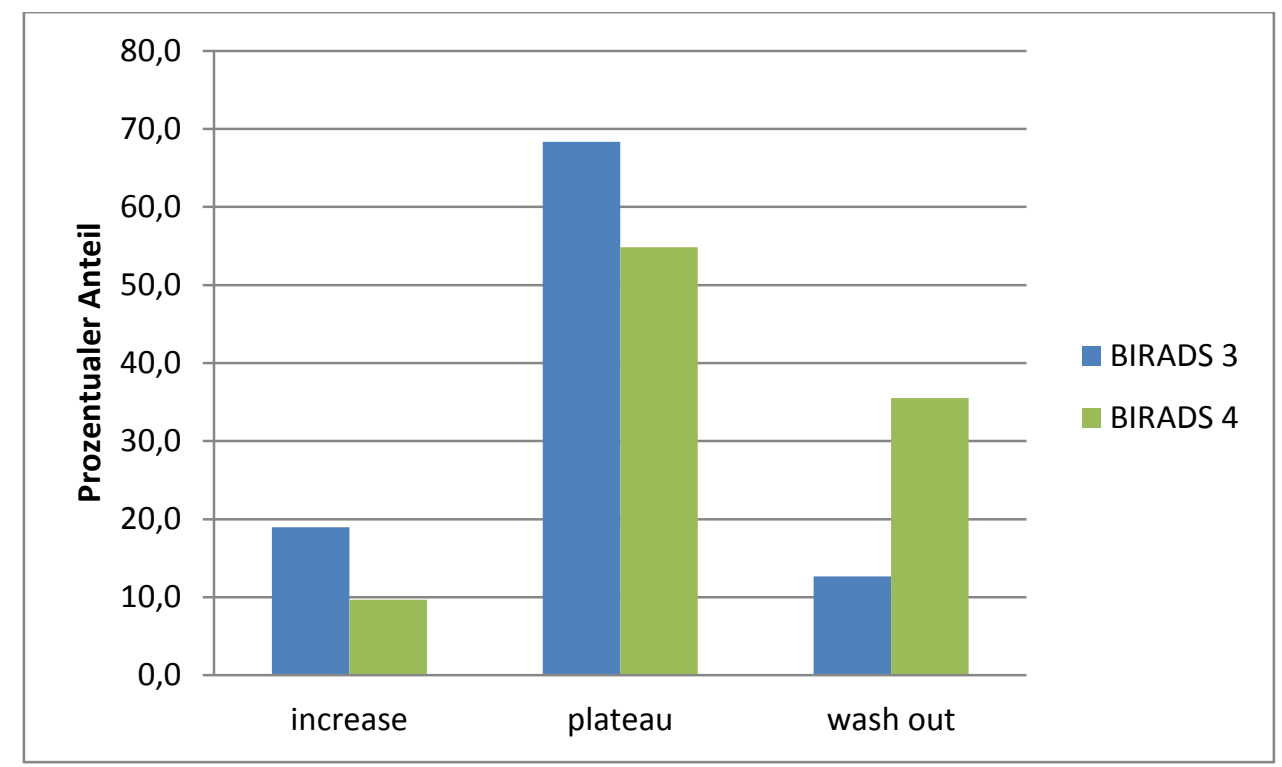

Abbildung 15: Postinitialer Signalverlauf der Herde in Abhängigkeit von der MRM-BIRADS-Kategorie.

Hinsichtlich des endotumoralen Wassersignals lag in der Kategorie MRM-BIRADS 3 in weniger als $10 \%$ ein hypointenses Verhalten vor, während sich der Rest annähernd gleichmäßig auf Iso- und Hyperintensität verteilte. In der Kategorie MRM-BIRADS 4 fand sich gehäuft ein isointenses Muster (47\%), gefolgt von Hypo- und Hyperintensität zu annähernd gleichen Anteilen (Abb. 16). 


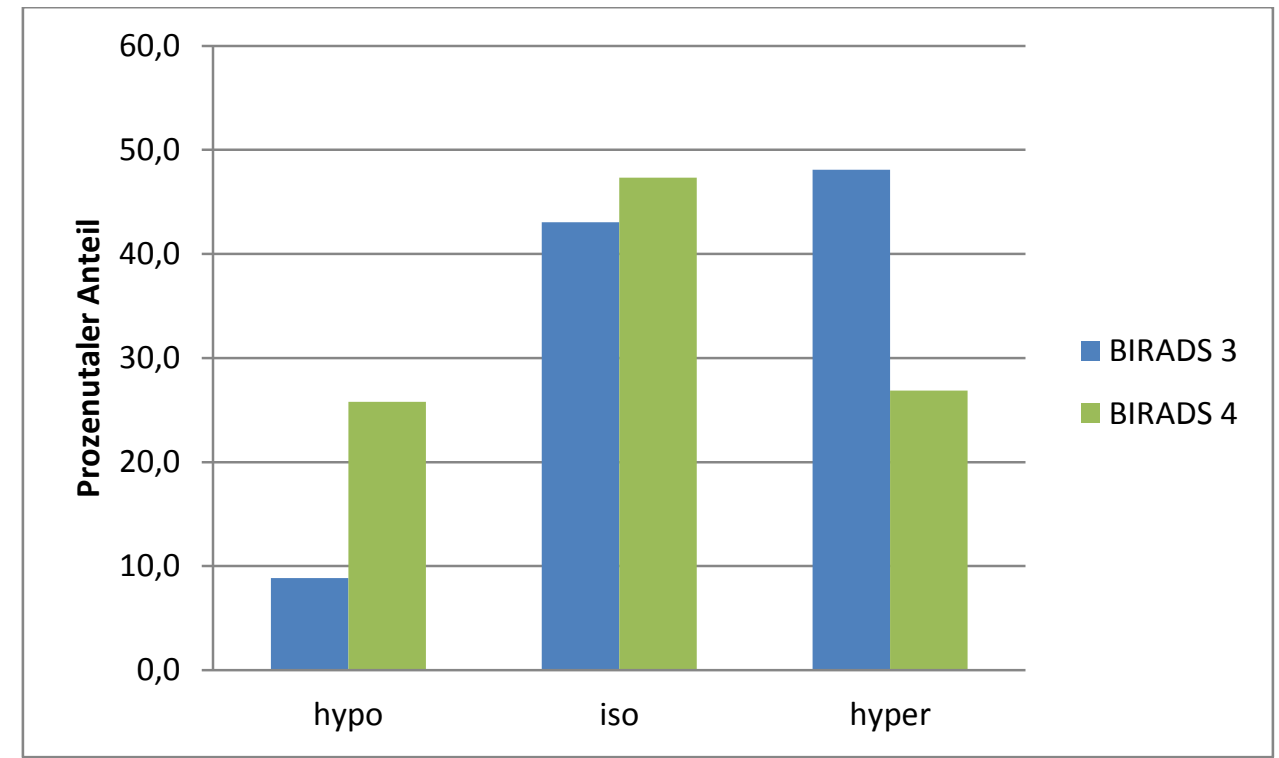

Abbildung 16: Wassergehalt der Herde in Abhängigkeit von der BIRADS-Kategorie.

Im Gesamtkollektiv fanden sich insgesamt kaum helle endotumorale Septierungen oder ein sog. „central enhancement". Diese waren allesamt beschränkt auf die Gruppe MRMBIRADS 4. Dunkle Septen waren in der Gruppe der Befunde der Kategorie MRMBIRADS 3 etwas häufiger als bei den MRM-BIRADS 4 Befunden (31\% versus 10\%) zu erkenne. In beiden Gruppen zeigten mehr als die Hälfte der Herdbefunde überhaupt keine Septierungen (Abb. 17). 


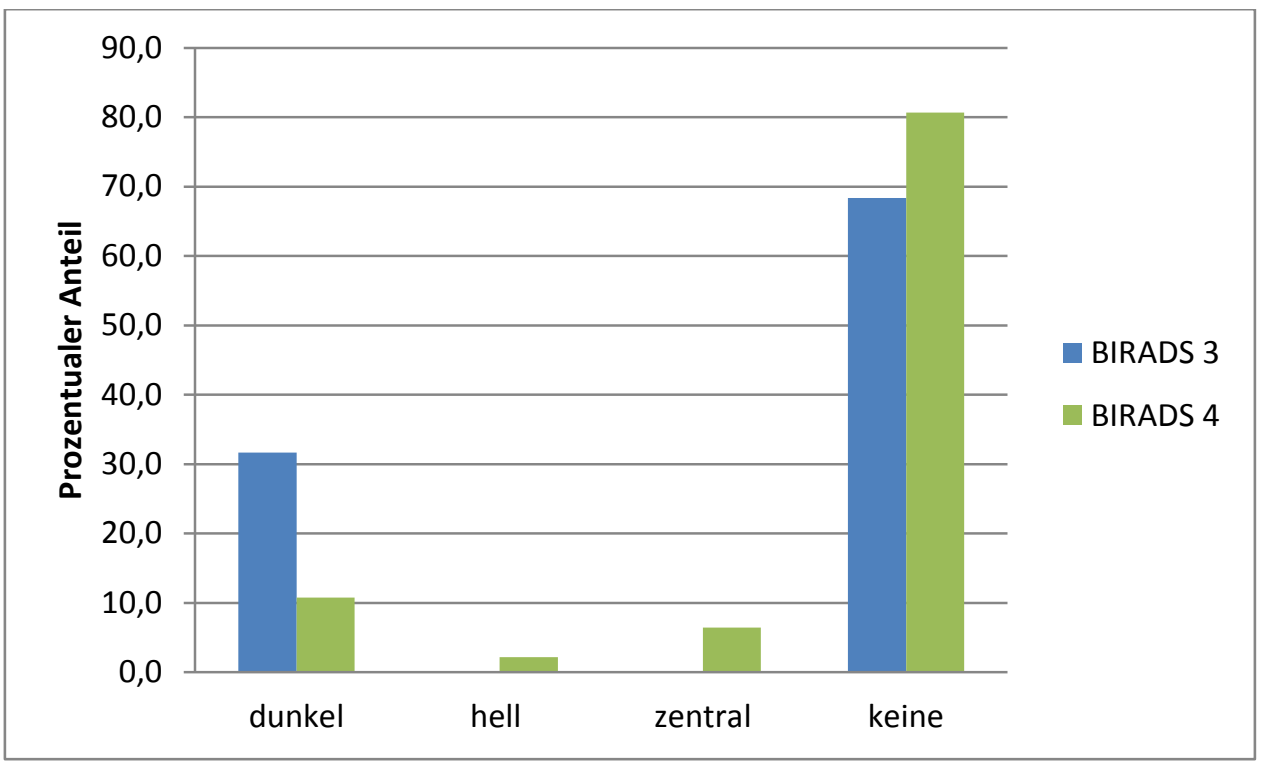

Abbildung 17: Endotumorale Septierungen der Herde in Abhängigkeit von der BIRADS-Kategorie.

In der Gruppe der Herdbefunde wiesen die meisten Befunde einen Göttingen-Score mit einem Gesamtwert von 3 (20\%), 4 (27\%) oder 5 (24\%) auf. Herdbefunde mit einem Punktwert von 1 (2\%), 7 (5\%) oder 8 (1\%) kamen selten vor (Abb. 18).

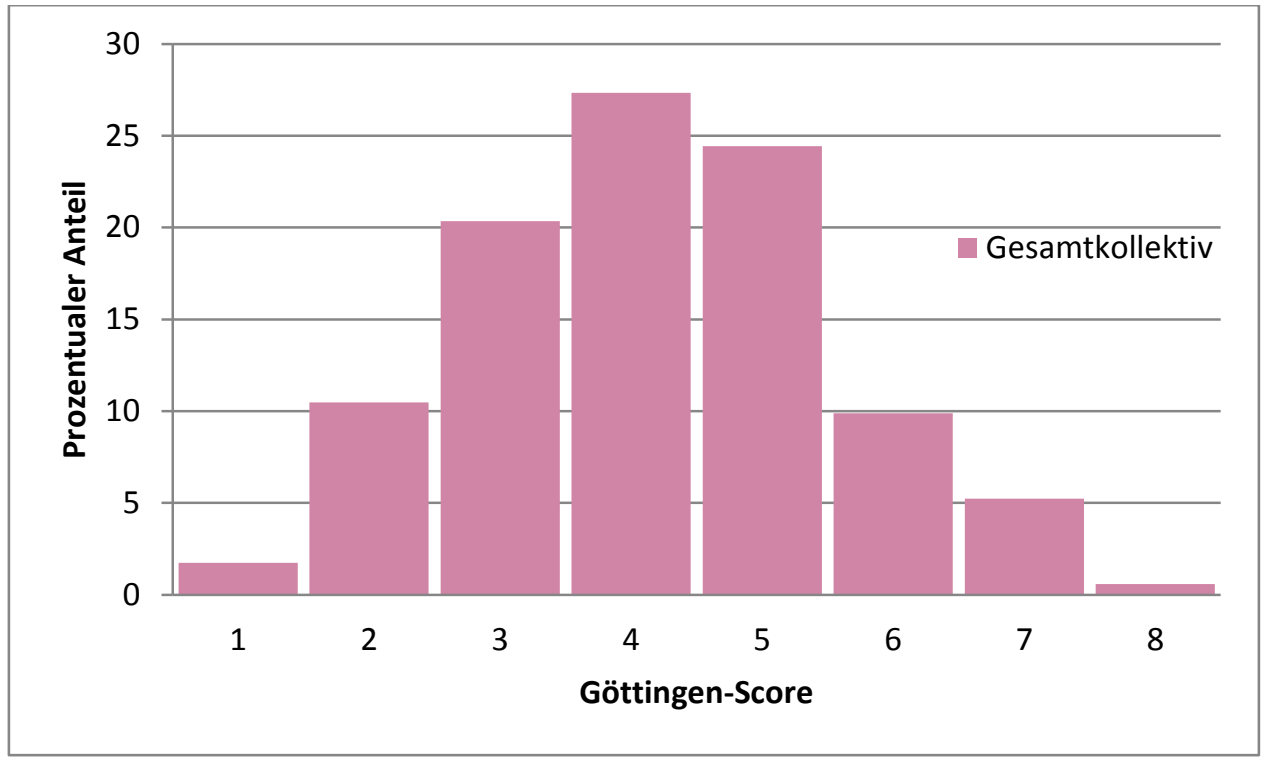

Abbildung 18: Prozentuale Verteilung der Punktwerte des Göttingen-Score für die Gesamtzahl aller Herde. 
Der Großteil der Befunde der Kategorie MRM-BIRADS 3 wies einen Punktwert zwischen 2 und 5 auf, wobei am häufigsten ein Score von $4(30,4 \%)$ vorlag. Eine Gesamtpunktzahl von 7 oder 8 Punkten kam nicht vor (Abb. 19).

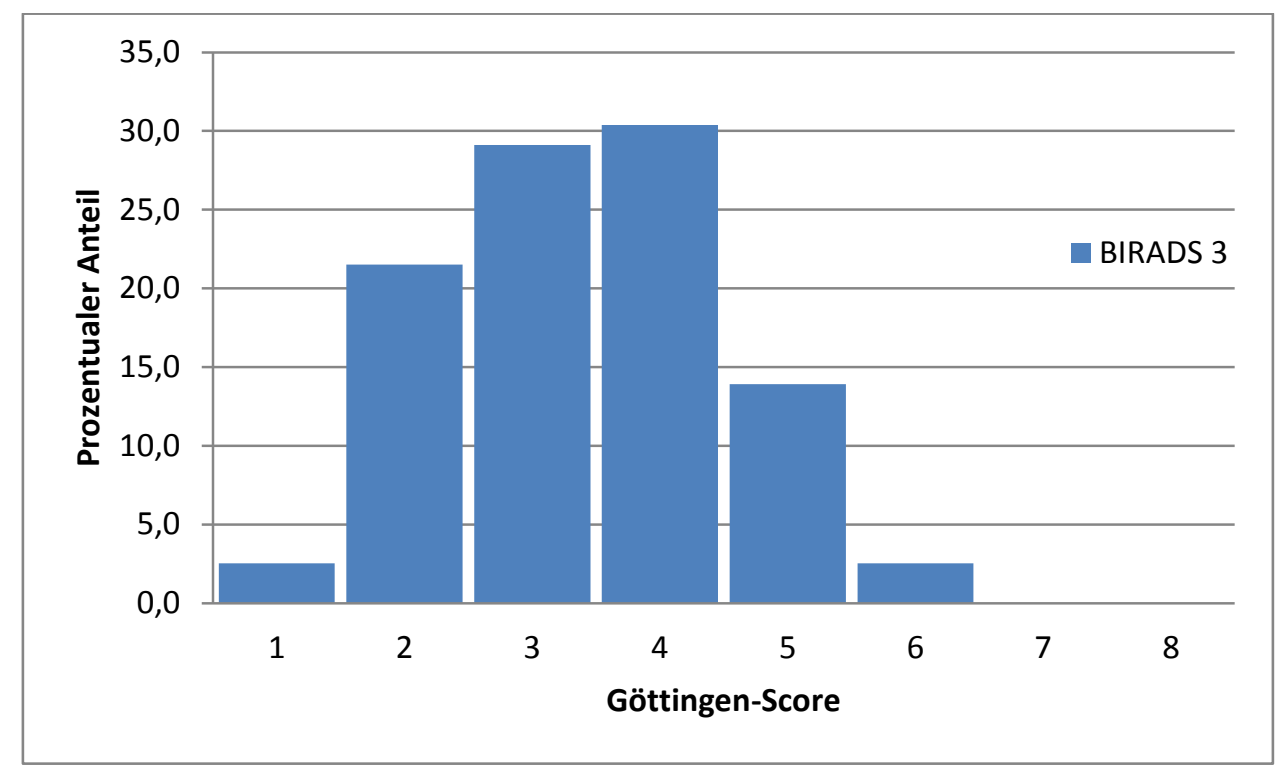

Abbildung 19: Prozentuale Verteilung der Gesamtpunkte des Göttingen-Score für die BIRADS-3Kategorie.

Befunde der Kategorie MRM-BIRADS 4 zeigten in etwa einem Drittel der Fälle eine Punktzahl von 5 auf, gefolgt von Punktwert 4, 6 und 3. Eine Gesamtpunktzahl von 1, 2 oder 8 lag in fast keinem Fall vor (Abb. 20). 


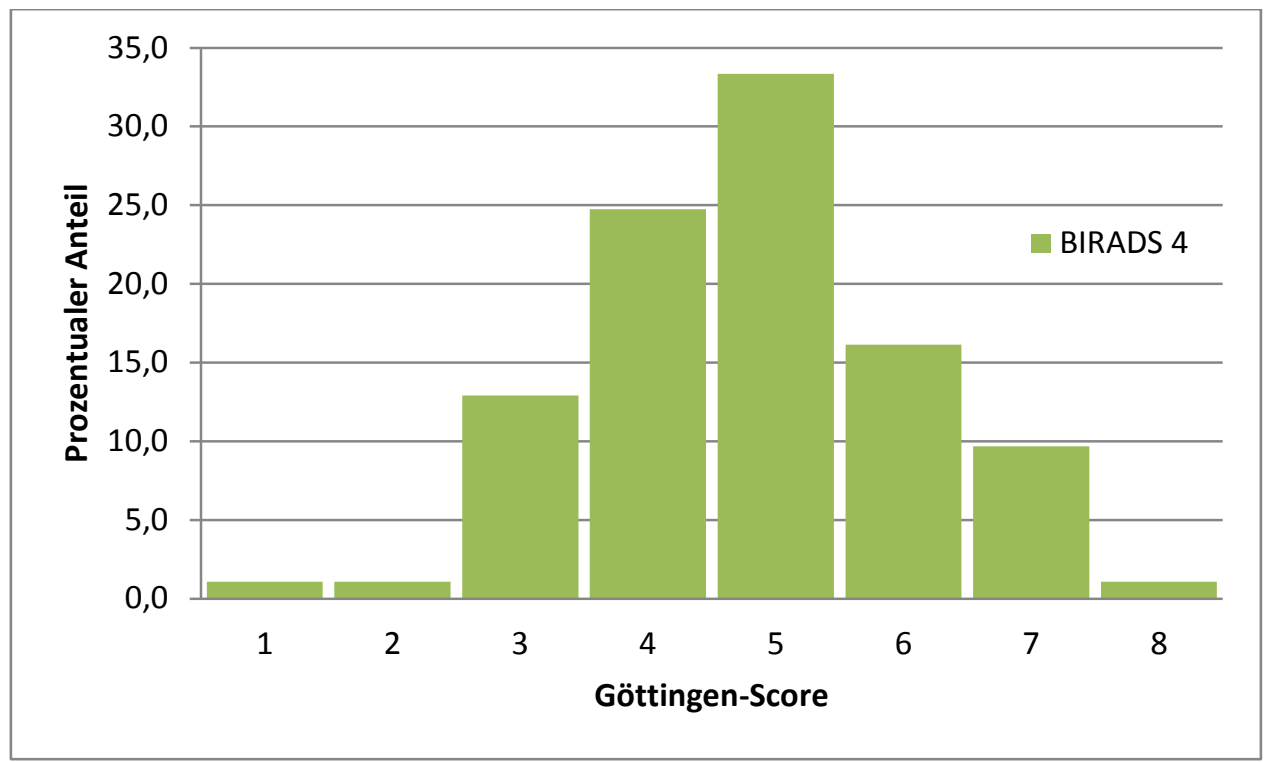

Abbildung 20: Prozentuale Verteilung der Gesamtpunkte des Göttingen-Score für die BIRADS-4Kategorie.

3.2.3 MR-tomografische Läsionsbeurteilung der NMLs

Die Abbildung 21 stellt die Größe der insgesamt 162 diagnostizierten NMLs dar. Es zeigte sich, dass in beiden BIRADS Kategorien die Mehrheit der NMLs zwischen 5 und $40 \mathrm{~mm}$ groß war. Mit jeweils 38,9\% war ein Ausmaß von 10-20mm in beiden Kategorien führend (Abb. 21).

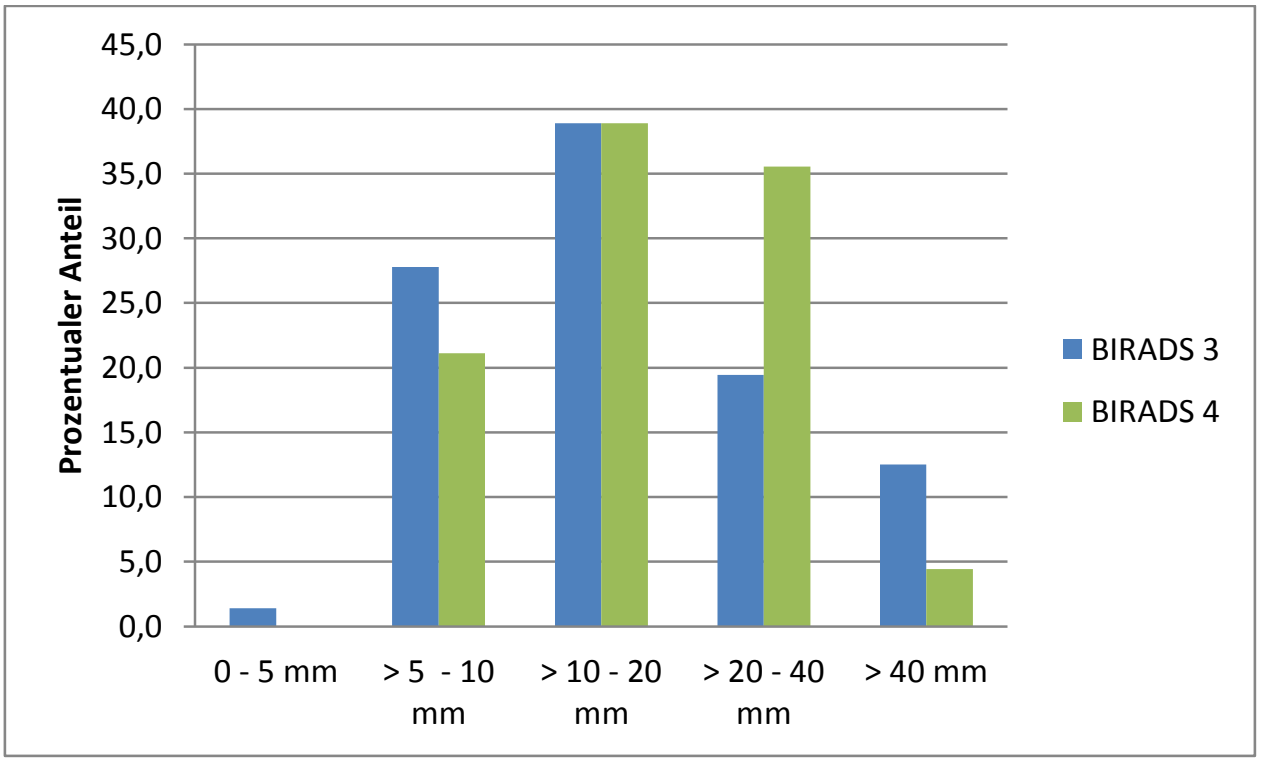

Abbildung 21: Größe der NMLs differenziert nach BIRADS-Kategorie. 
Hinsichtlich der Anreicherungsform der kontrastmittelanreichernden NMLs fand sich für alle Muster eine ausgewogene Verteilung zwischen den beiden Befundkategorien MRMBIRADS 3 und MRM-BIRADS 4. Für beide MRM-BIRADS-Kategorien fanden sich hierbei in etwa der Hälfte der Fälle regionale Anreicherungen, gefolgt von linearen und segmentalen Konfigurationen (Abb. 22).

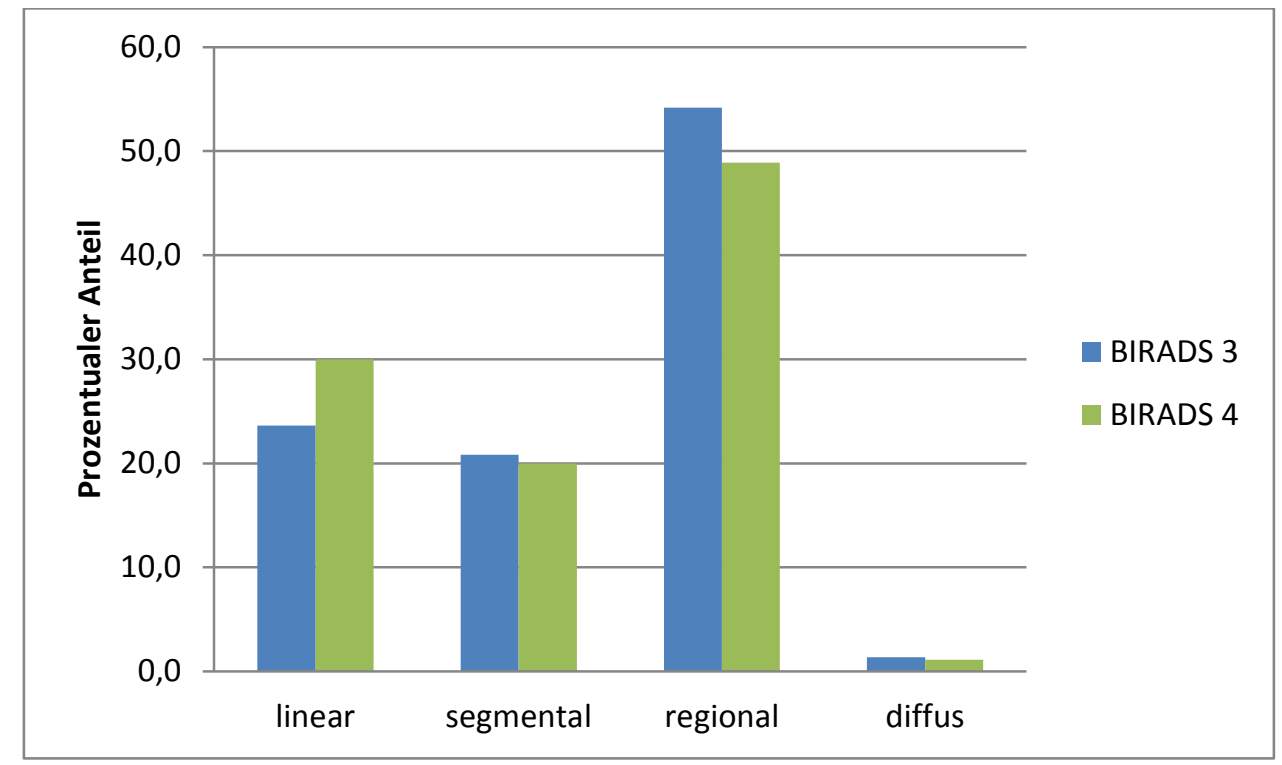

Abbildung 22: Anreicherungsform der NMLs differenziert nach BIRADS-Kategorie.

\subsection{Verlaufskontrolle der Studienpopulation}

Von den insgesamt 196 Patientinnen, deren Erstuntersuchung eine Befundkonstellation der MRM-BIRADS-Kategorie 3 ergab, stellten sich 175 (89\%) zu einem Kontrolltermin im Diagnostischen Brustzentrum Göttingen vor. Durchschnittlich erschienen die Patientinnen 8,2 Monate nach der Voruntersuchung, wobei dies frühestens nach 2 Monaten und spätestens nach 48 Monaten geschah. 107 Patientinnen stellten sich ohne ein Anschreiben seitens des Diagnostischen Brustzentrum Göttingen zu einem Kontrolltermin vor, während 63 erst nach Aufforderung zur Kontrolle erschienen. Eine histologische Abklärung erfolgte bei 23 Patientinnen. 6 dieser Biopsien stellten sich als maligne (histologische B-Klassifikation: B5a, B5b) heraus, 5 als Tumoren unklarer Dignität (histologische B-Klassifikation: B3) und 12 als benigne (histologische B- 
Klassifikation: B1, B2)(Abb. 23). In der Gruppe mit unklarem biologischen Potential fanden sich im weiteren Verlauf $(n=2)$ oder in der anschließenden offenen Biopsie $(n=3)$ keine Karzinome, so dass die Quote der malignen Prozesse in der Kategorie MRMBIRADS 3 insgesamt 3,1\% betrug.

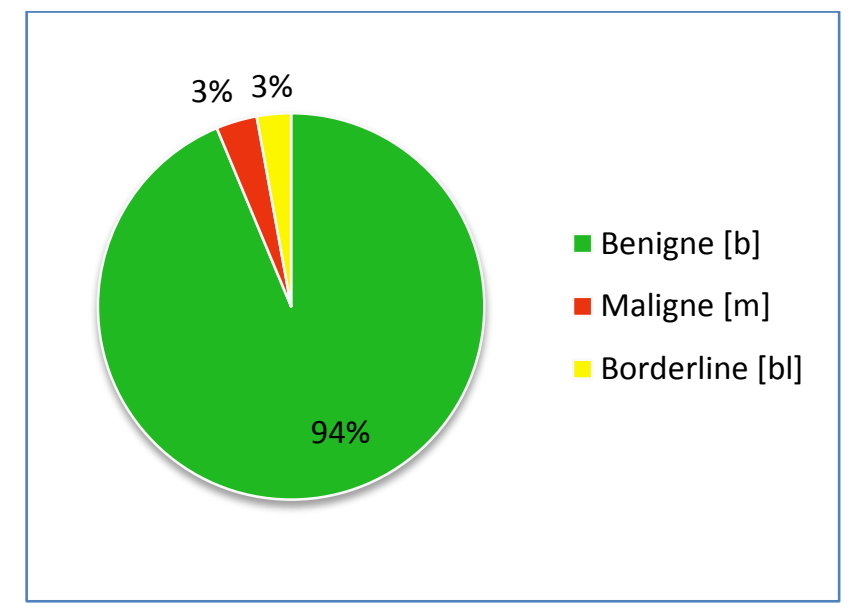

Abbildung 23: Dignität der Befunde von 196 Patienten mit Befunden der Kategorie MRM-BIRADS 3 (23 Befunde histologisch verifiziert).

Wie von der Arbeitsgruppe des American College of Radiology empfohlen, wurden alle 198 als MRM-BIRADS 4 typisierten Patientinnen vakuumbioptisch abgeklärt. 107 der Läsionen stellten sich als benigne, 64 als maligne und 27 als Borderline-Prozesse heraus (Abb. 24). Die Gruppe der Borderline-Läsionen setzte sich zusammen aus Papillom/Papillomatose $(n=16)$, ADH bzw. FEA $(n=4)$, LIN $(n=4)$ und radiärer Narbe $(n=3)$. Im Rahmen einer gemeinsamen radiologisch-pathologischen Konferenz wurde bei 16 dieser 27 Borderline-Läsionen eine weitergehende Abklärung in Form einer operativen Befundentfernung festgelegt. Hierbei fanden sich insgesamt 5 Karzinome, so dass in der Gruppe BIRADS 4 insgesamt 69 maligne Tumoren (34,8\%) vorlagen. 


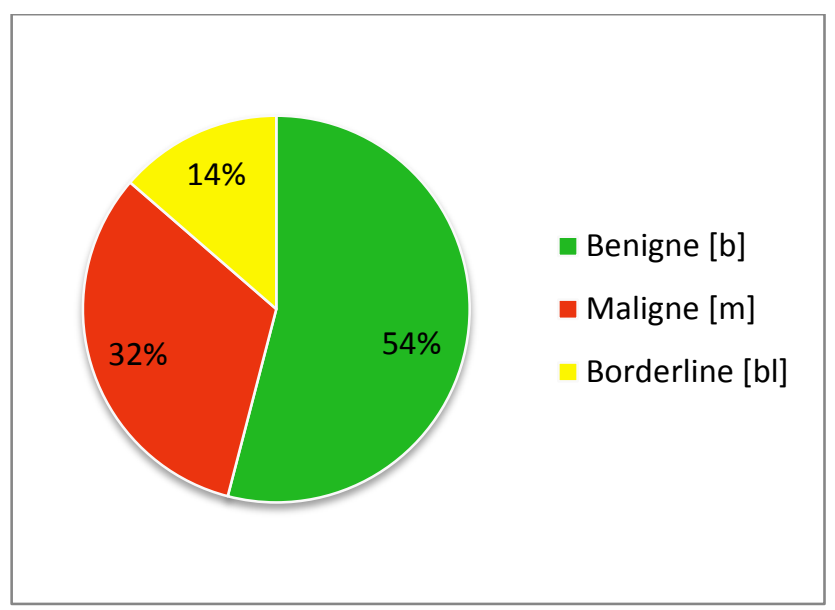

Abbildung 24: Histologische Klassifikation der Befunde von 198 Patienten mit Befund der Kategorie MRM-BIRADS 4.

Im Folgenden werden - unabhängig von der primären Zuordnung zu einer BIRADSGruppe - alle Frauen mit unverändertem Befund im Verlauf oder histologisch gesicherter Gutartigkeit ( $\mathrm{n}=292$ ) als Gruppe der gesunden Frauen (Gesunde) zusammengefasst. Frauen mit histologisch gesichertem Mammakarzinom ( $n=70)$ werden - unabhängig von der primären Kategorisierung - als Gruppe der Patienten mit Mammakarzinom (Kranke) zusammengefasst. Die verbleibenden Frauen mit histologisch nachgewiesener Läsion mit biologisch unklarem Potential $(n=32)$ bilden eine dritte separate Gruppe (Borderline).

Betrachtet man den Typus der pathologischen Kontrastmittelanreicherung, so traten in der Gruppe der Gesunden zu annähernd gleichen Teilen Herdbefunde (44,2\%) und NMLs (43,5\%) auf. Foki fanden sich in 12,3\%. In der Gruppe der Kranken dominierten die Herde mit 57,4\%, gefolgt von NMLs (41,2\%). Foki waren nur in 1,5\% der Fälle zu finden. Im Borderline-Kollektiv fanden sich überwiegend NMLs $(43,8 \%)$ vor den Herdbefunden. Foki fanden sich in dieser Gruppe seltener (Abb. 25). 


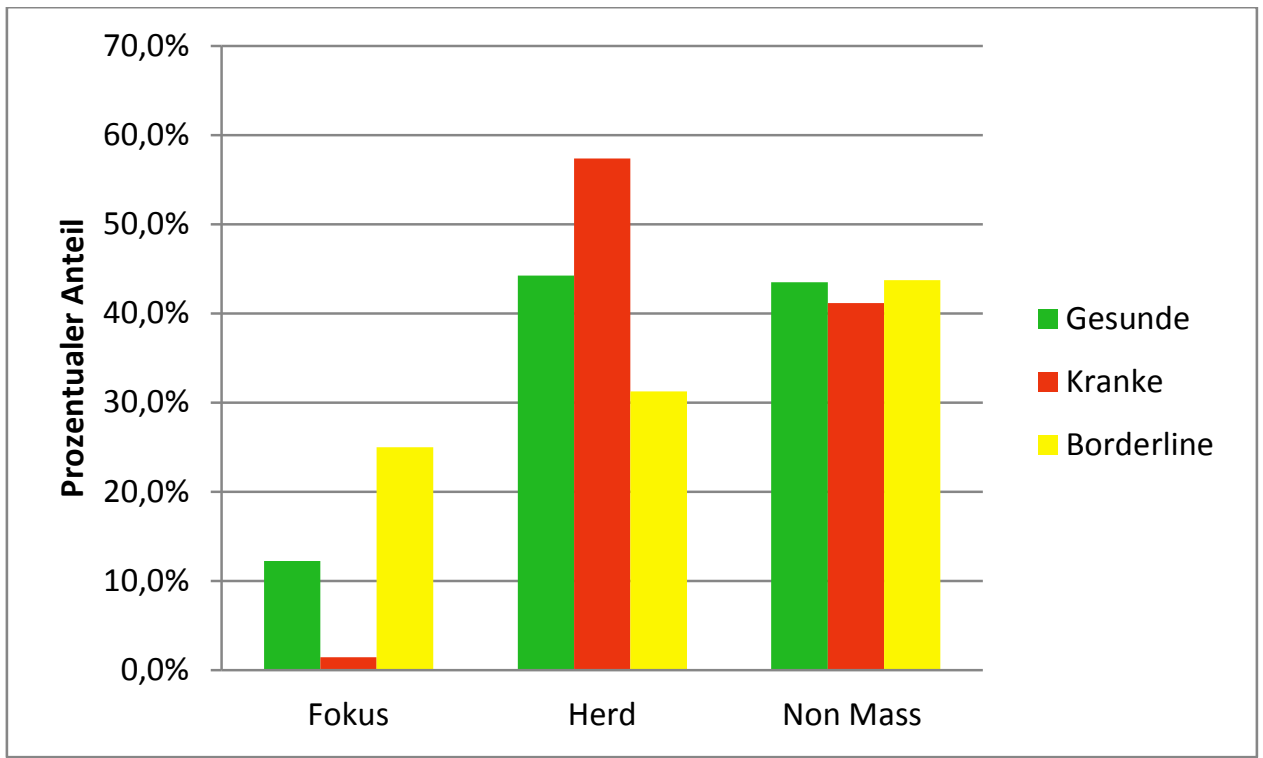

Abbildung 25: Verteilung der 3 Typen anreichernder Läsionen in Abhängigkeit von der Dignität des Befundes.

Die Mehrzahl der kategorisierten Foki betrug 3 und 4mm. Mit Zunahme der Fokusgröße fielen zunehmend mehr Befunde in die Gruppe der Patienten mit Mammakarzinom bzw. mit Boderlineläsion. In der Gruppe der Kranken wiesen alle Foki einen Durchmesser von $4 \mathrm{~mm}$ auf (Abb. 26).

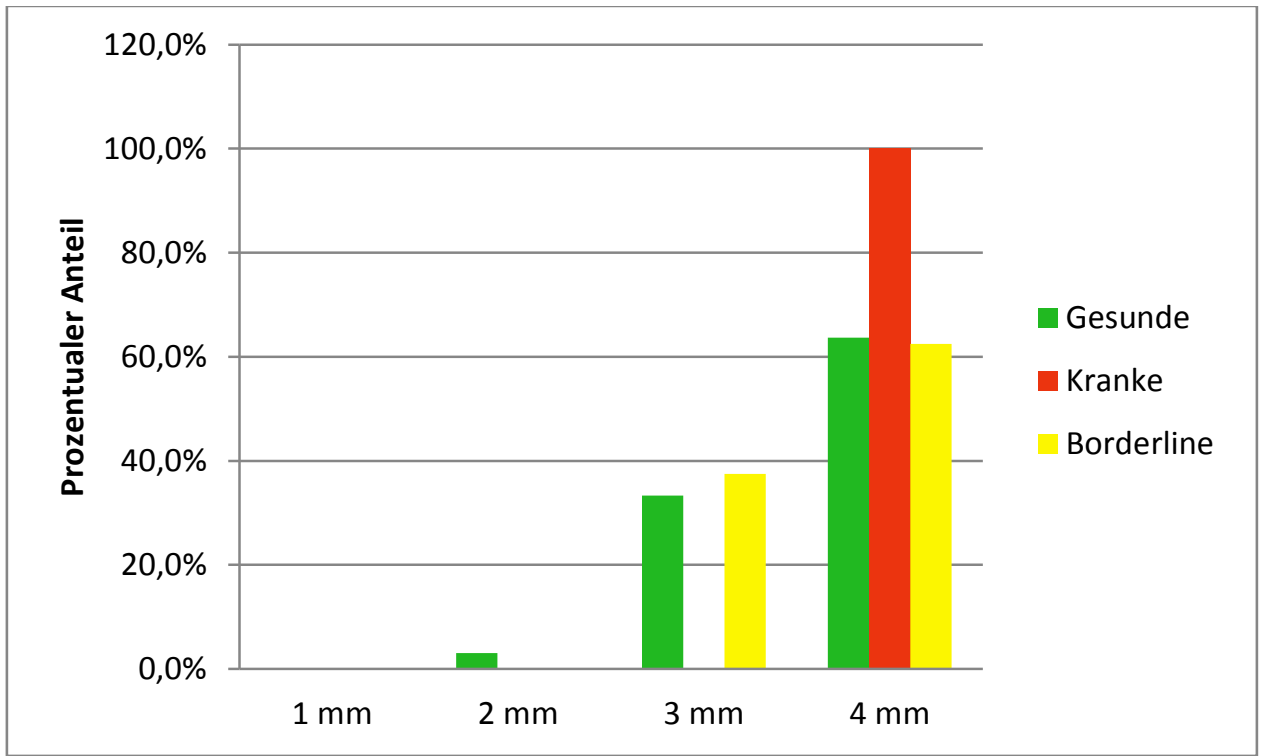

Abbildung 26: Fokusgröße in Abhängigkeit von der Dignität der Läsion. 
In allen drei Gruppen verteilten sich die Herdbefunde zu über 75\% auf eine Größe zwischen 5 und $10 \mathrm{~mm}$. In annähernd identischer Weise fanden sich in allen 3 Gruppen etwa $10 \%$ der Herde in einer Größenordnung zwischen 11 und $15 \mathrm{~mm}$. Einige wenige Karzinome wiesen eine Größe über 15mm auf (Abb. 27).

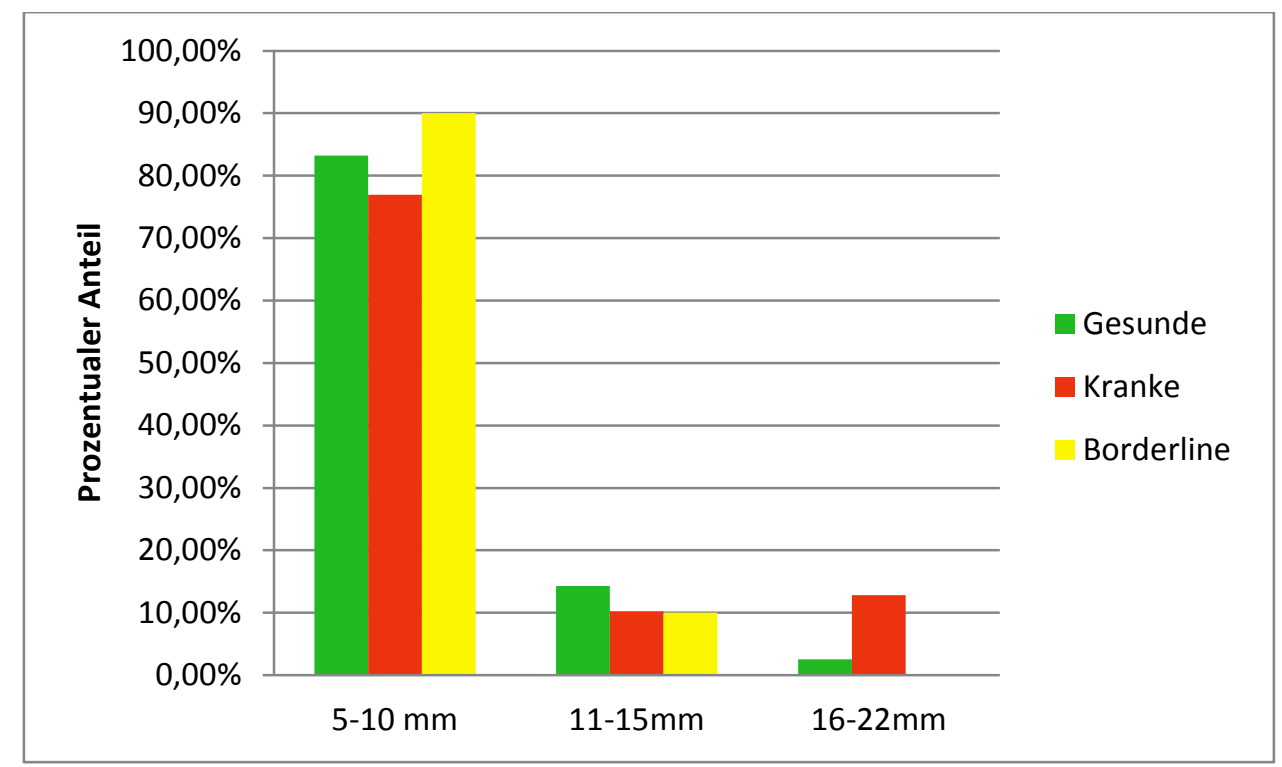

Abbildung 27: Größe der Herdbefunde in Abhängigkeit von der Dignität.

In der Gruppe der Gesunden traten mit über 72\% hauptsächlich ovale und runde Formen bei den Herdbefunden auf. Eine ähnlich hohe Rate $(74,3 \%)$ fand sich bei den bösartigen Tumoren für runde (48,7\%) und irreguläre Formen $(25,6 \%)$ der Herde. Läsionen vom Typus Borderline wiesen in etwas weniger als zwei Drittel der Fälle eine runde Konfiguration auf. Hier war die Form der Herdbefunde - wie auch bei den gutartigen Veränderungen in 10\% irregulär (Abb. 28). 


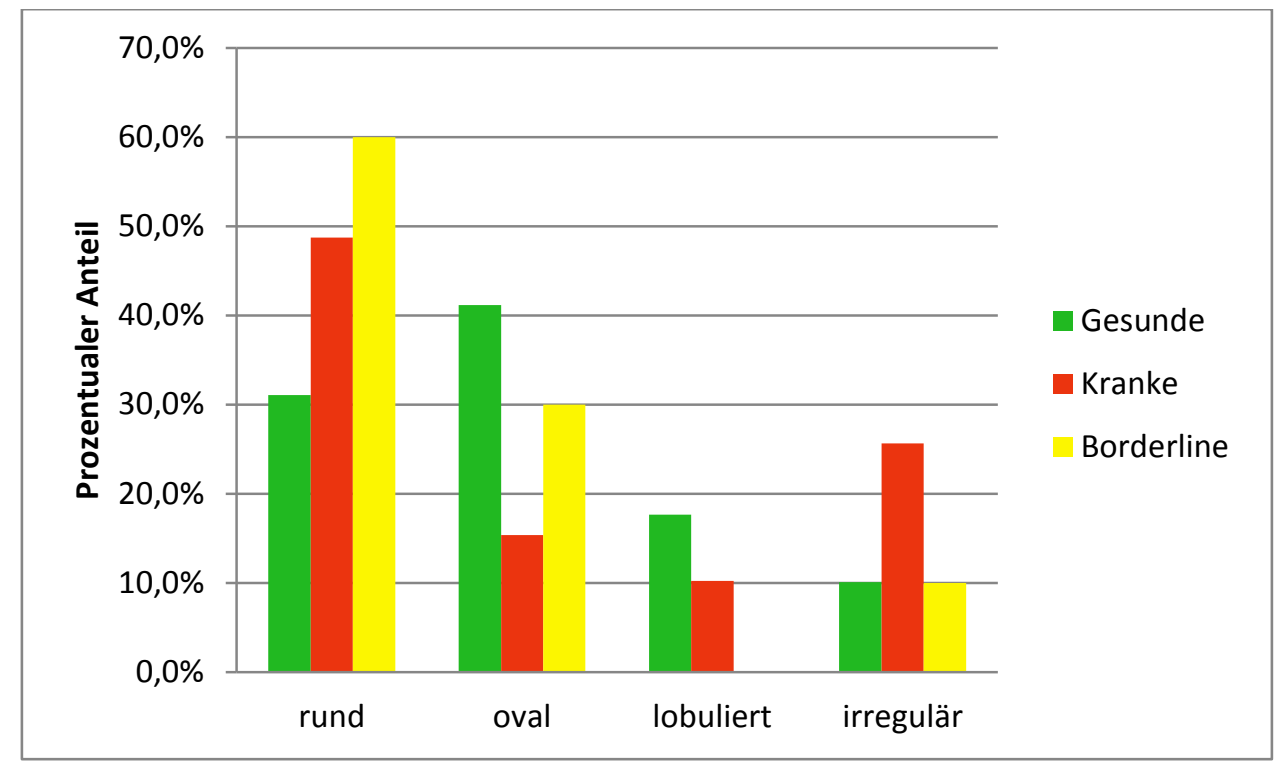

Abbildung 28: Form der Herdbefunde in Abhängigkeit von der Dignität.

Die äußere Kontur der Herdbefunde stellte sich in der Gruppe der Gesunden zu fast gleichen Teilen als glatt (53,8 \%) und unscharf (46,2 \%) dar. In der Gruppe der Erkrankten zeigte der wesentliche Anteil (87,2\%) eine unscharfe Begrenzung auf. Auch in der Borderline-Gruppe stellten sich mit $60 \%$ mehr unscharfe als glatte (40\%) Läsionskonturen dar (Abb. 29).

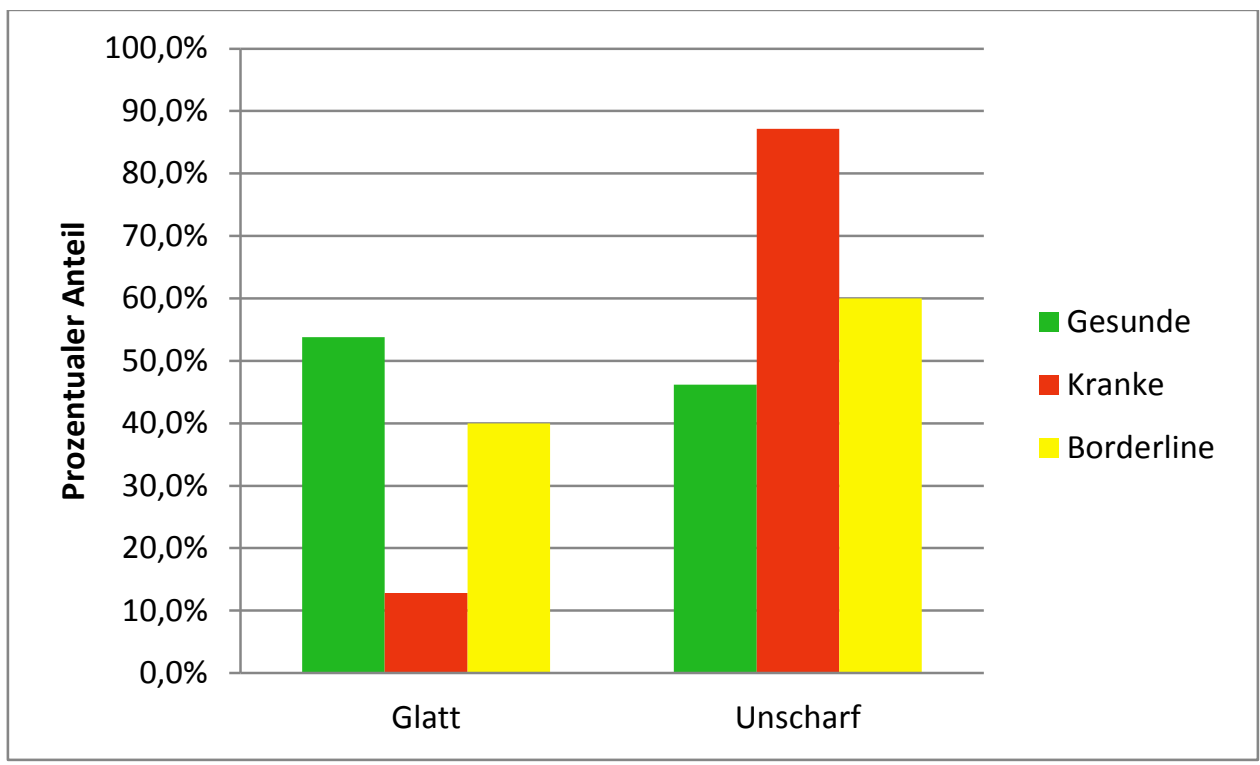

Abbildung 29: Begrenzung der Herdbefunde in Abhängigkeit von der Dignität. 
Bei der Betrachtung der räumlichen Verteilung des Kontrastmittels innerhalb der Herdbefunde zeigte sich in allen drei Gruppen vorrangig ein inhomogenes Binnenmuster. Ein homogenes Verteilungsmuster stellte sich mit $34,5 \%$ häufig bei den Gesunden und mit 7,7\% selten bei den Kranken dar. Bei mehr als einem Drittel der bösartigen Befunde fand sich ein Ringenhancement, während diese Quote bei den gutartigen Herdbefunden mit $<18 \%$ deutlich geringer war (Abb. 30).

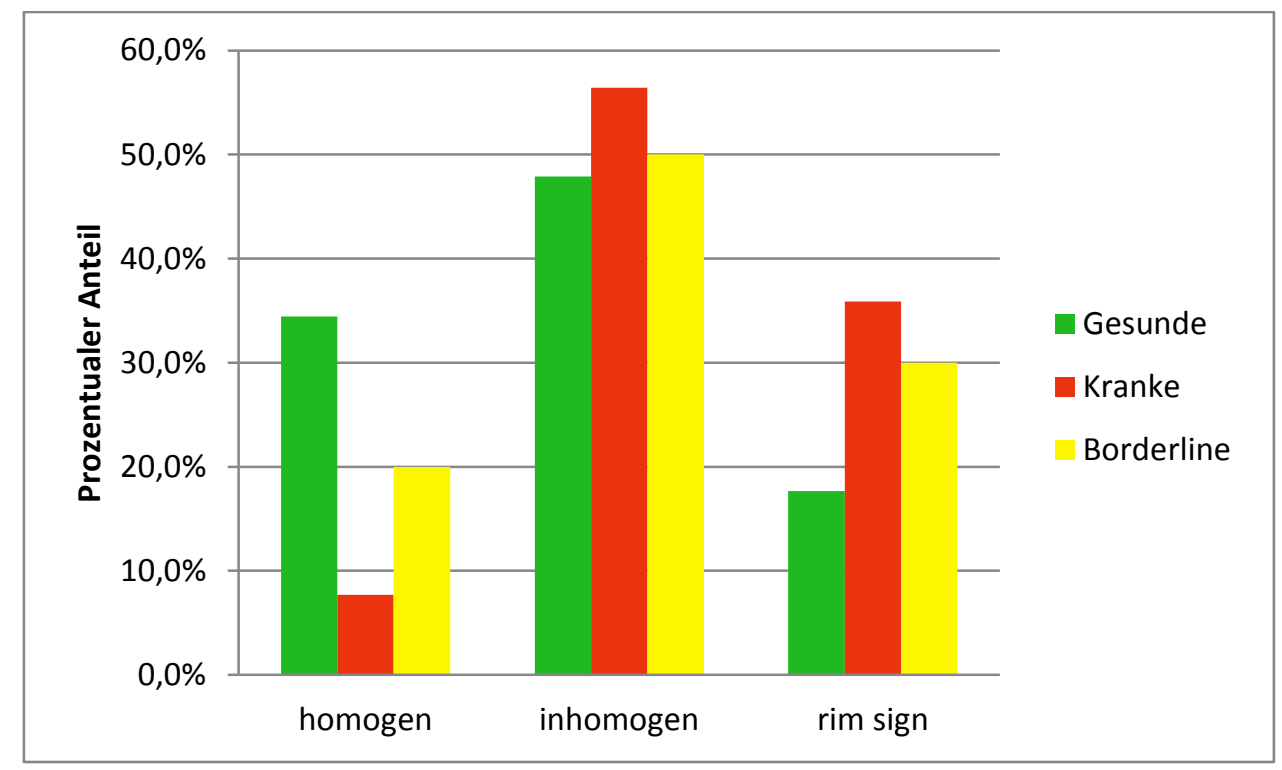

Abbildung 30: Endotumorales Verteilungsmuster des Kontrastmittels innerhalb der Herdbefunde in Abhängigkeit von der Dignität.

Hinsichtlich des Ausmaßes des initialen Signalanstieges ließen sich für gut- und bösartige Herdbefunde keine Unterschiede erkennen. Einer mäßigen (50-100\%) oder starken Ausprägung (>100\%), kam für beide Dignitäten in etwa $50 \%$ vor. Hiervon setzten sich die Borderline-Läsionen mit einem mäßigen Anstieg in etwa $80 \%$ der Fälle ab. Ein geringer initialer Signalanstieg kam so gut wie in keiner der 3 Gruppen vor (Abb. 31). 


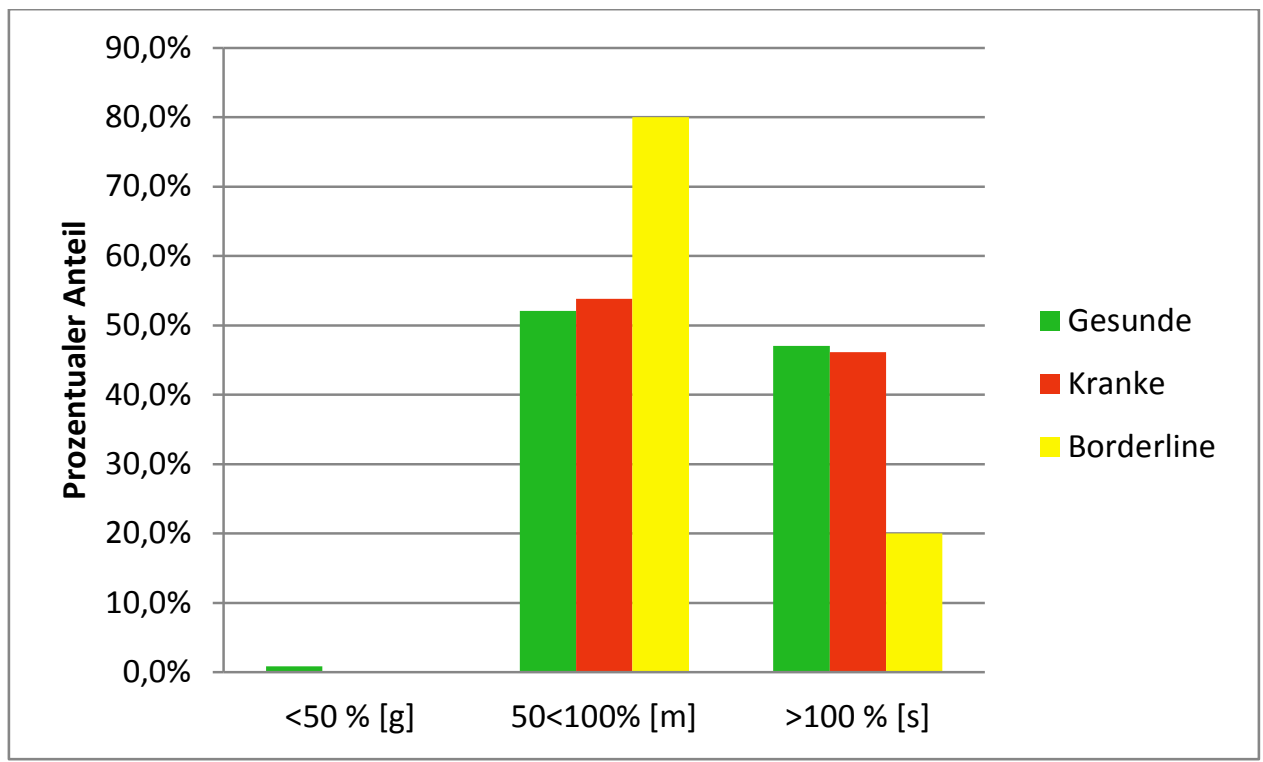

Abbildung 31: Initialer Signalanstieg der Herdbefunde in Abhängigkeit von der Dignität.

Bezüglich der postinitialen Phase dominierten in der Gruppe der gutartigen Befunde das Plateauphänomen (65,5\%), während ein wash out mit 16,8\% am seltensten zu beobachten war. Anders stellte sich die Situation bei den bösartigen Befunden dar: Hier fand sich in je fast $50 \%$ der Fälle ein Plateau- oder ein Auswaschphänomen. Die Borderline-Läsionen zeigten ausschließlich ein Plateau (60\%) oder ein wash out (40\%) (Abb. 32).

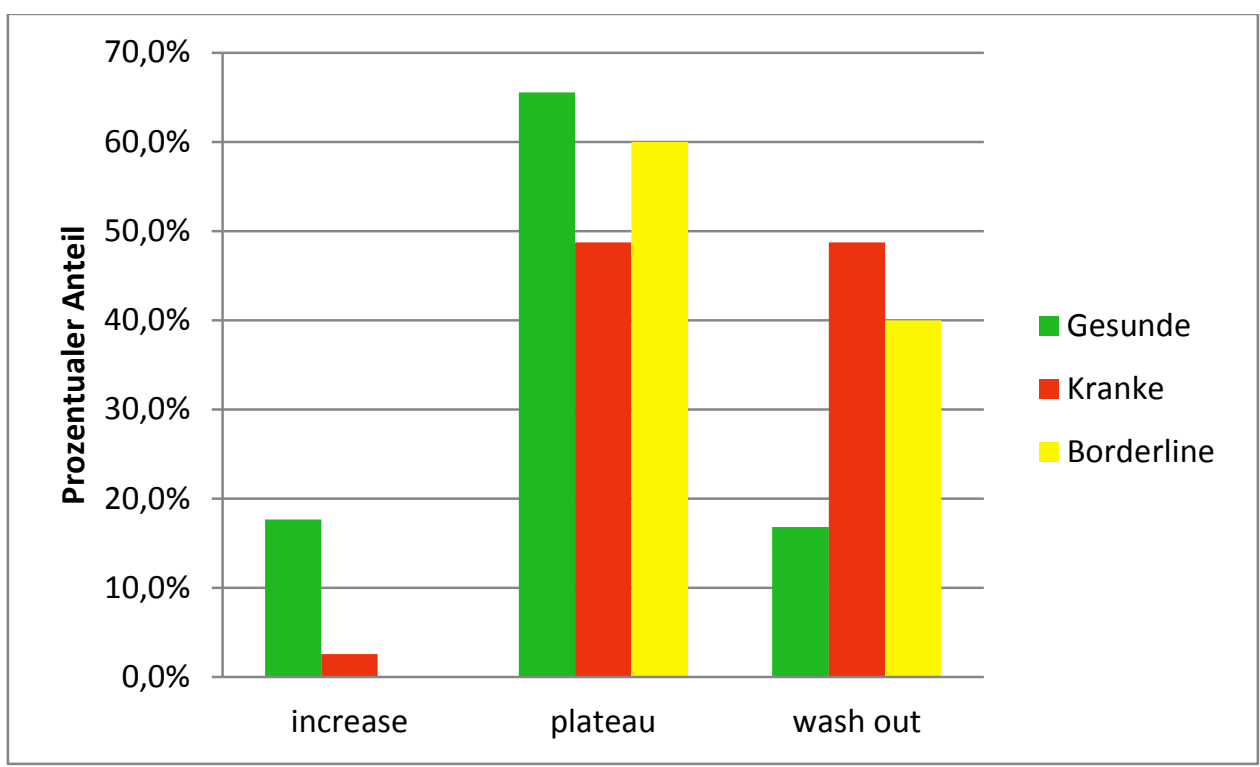

Abbildung 32: Postinitialer Signalanstieg der Herdbefunde in Abhängigkeit von der Dignität. 
Gutartige Herdbefunde wiesen in etwa $85 \%$ der Fälle einen im Vergleich zum umliegenden Drüsengewebe gleichen oder erhöhten Wassergehalt auf. Auch die Borderline-Läsionen hatten überwiegend (90\%) ein iso- oder hyperintenses Signal in der wassersensitiven Messung. Dieser Effekt drehte sich bei den bösartigen Prozessen um: Hier fand sich in weniger als $15 \%$ der Fälle ein erhöhter endotumoraler Wassergehalt, während über $85 \%$ dieser Tumoren hypo- oder isointens imponierten (Abb. 33).

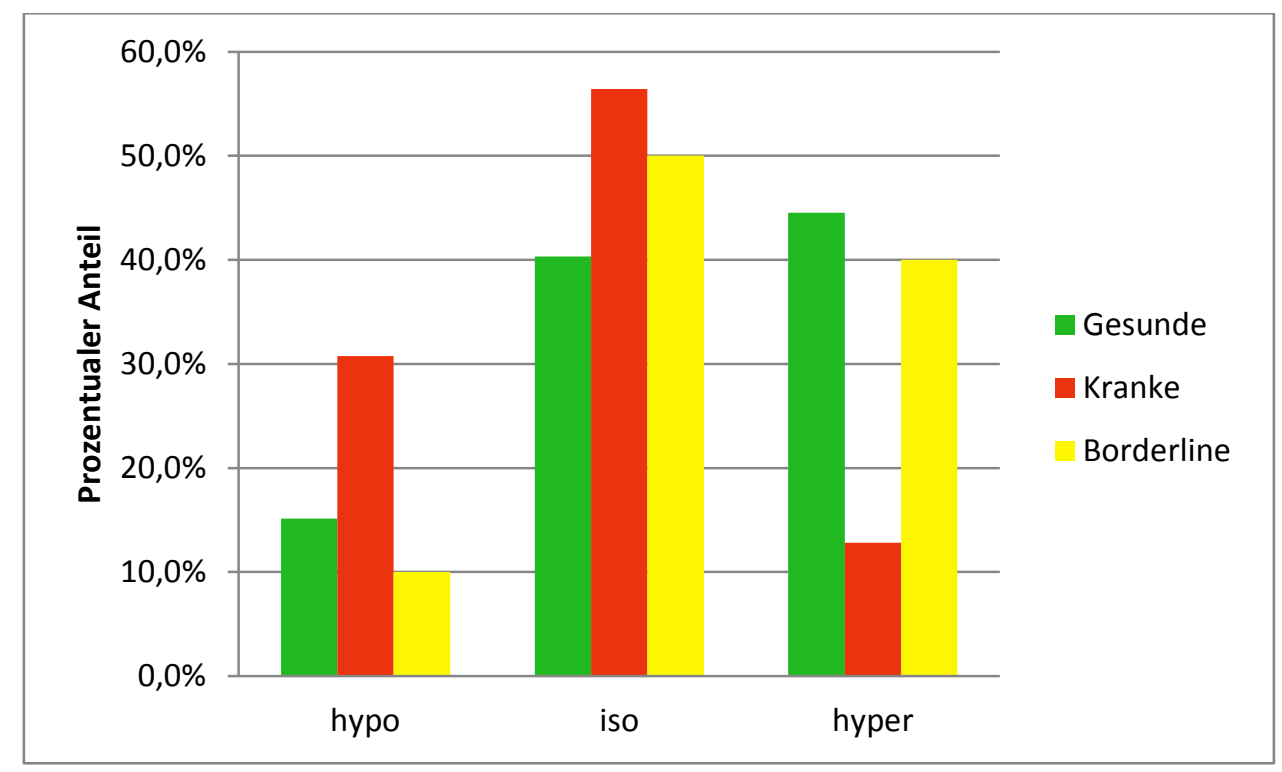

Abbildung 33: Wassergehalt innerhalb der Herdbefunde in Abhängigkeit von der Dignität.

Bei der Betrachtung der endotumoralen Septierungen zeigte sich, dass in allen drei Gruppen der wesentliche Anteil der Herdbefunde keine Septierungen aufwies. Die entsprechenden Quoten lagen zwischen 70 und 90\%. Helle Septierungen fanden sich mit $25 \%$ bevorzugt bei den Gesunden, aber auch zu 10\% bei den bösartigen Tumoren (Abb. 34). 


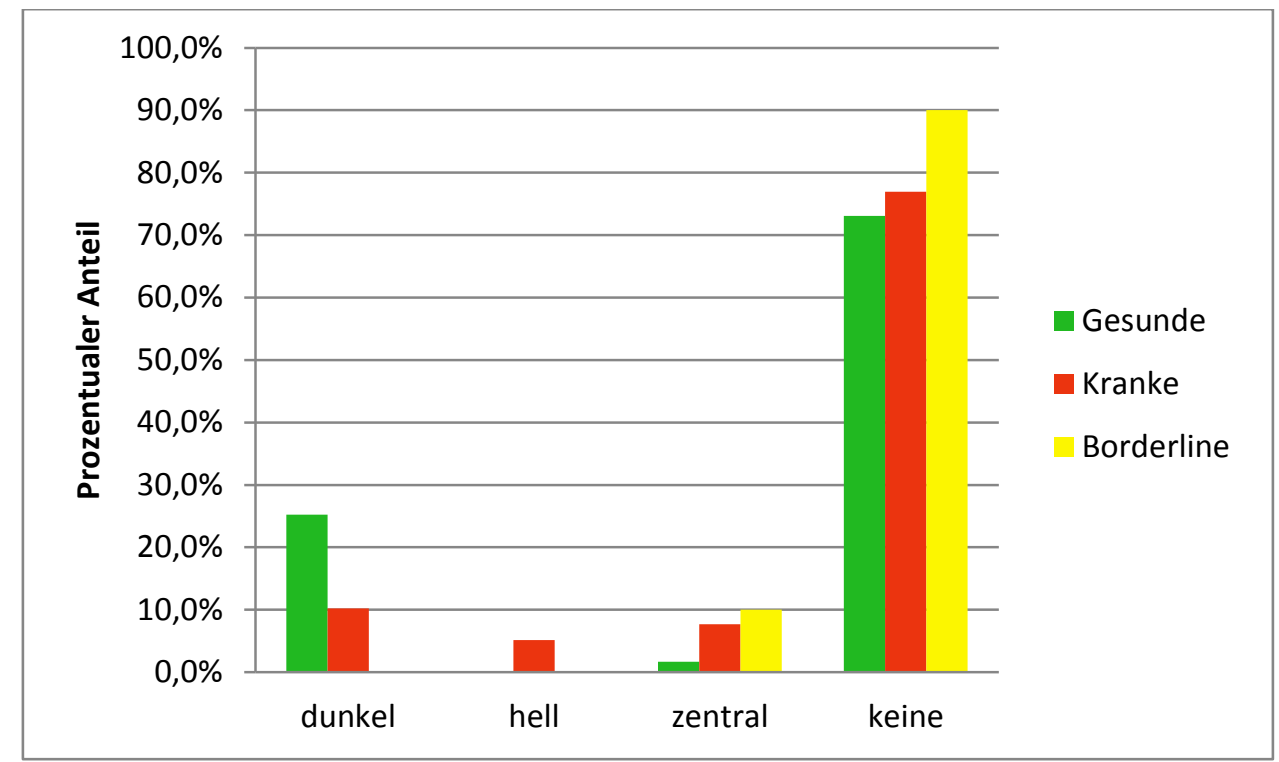

Abbildung 34: Endotumorale Septierungen der Herdbefunde in Abhängigkeit von der Dignität.

Nach Berechnung der jeweiligen Gesamtpunktewerte gemäß Göttingen-Score ergab sich für die gutartigen Befunde eine Normalverteilungskurve mit Maximum im Bereich von 4 Punkten $(29,4 \%)$, gefolgt von 3 Punkten $(27,7 \%)$ und 5 Punkten $(21,8 \%)$. Weniger als $10 \%$ der gutartigen Befunde hatte einen Score über 5 . Dieses Maximum verschiebt sich in der Gruppe der bösartigen Befunde um 2 Punkte nach rechts: Herdbefunde mit 5 oder 6 Punkten traten hier in je 28,2\% auf, gefolgt von 4 Punkten $(20,5 \%)$ und 7 Punkten (15,4\%). Keiner der bösartigen Tumoren hatte einen Score unter 3. Bei den Borderline-Läsionen lagen in 90\% der Fälle 4 oder 5 Punkte vor (Abb. 34). 


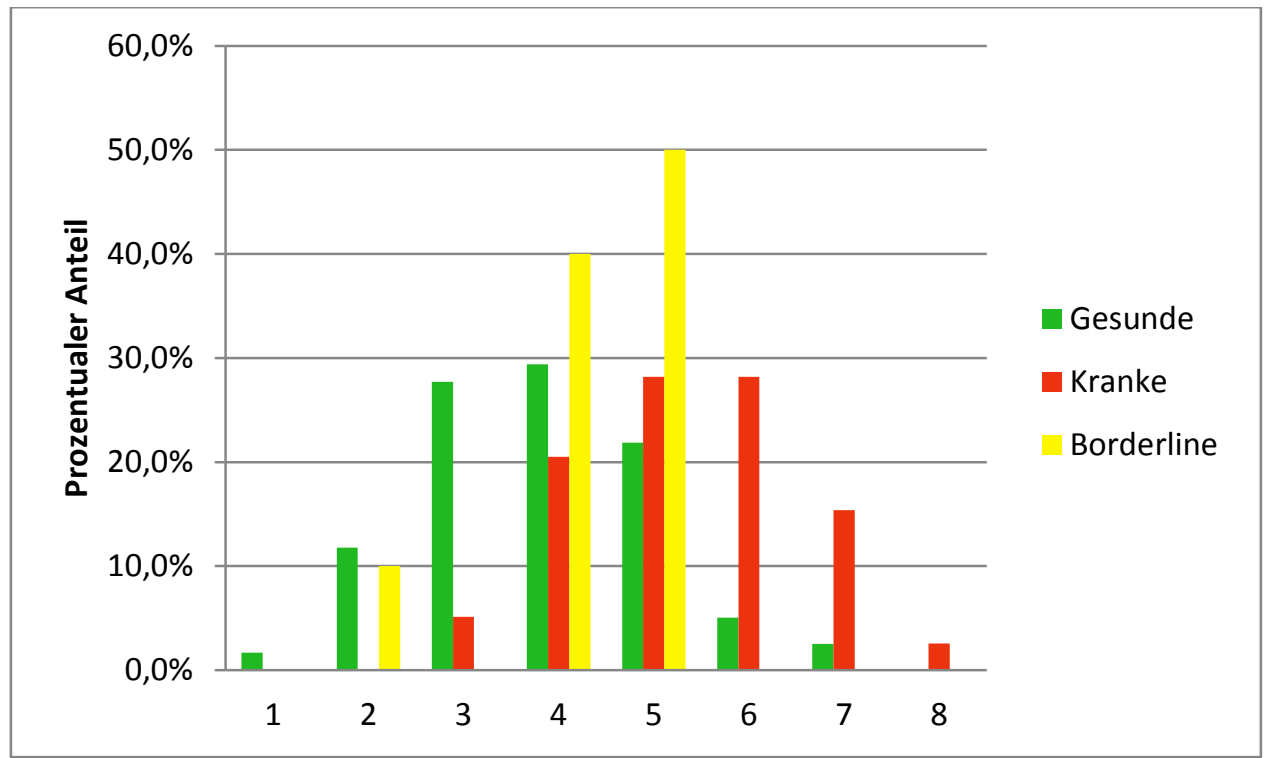

Abbildung 35: Punktwerte des Göttingen-Score der Herdbefunde in Abhängigkeit von der Dignität.

Bezüglich der Ausdehnung der NMLs fand sich in der Gruppe der gutartigen Läsionen eine ausgewogene Verteilung zwischen Befundgrößen von $5 \mathrm{~mm}$ bis $40 \mathrm{~mm}$. Bösartige Läsionen traten in über $80 \%$ der Fälle in Dimensionen zwischen 10 und $40 \mathrm{~mm}$ auf, während nur $10 \%$ kleiner als $10 \mathrm{~mm}$ waren. Auch die Borderline-Läsionen lagen in einem sehr geringen Anteil (15\%) in einer Größenordnung <10mm, während über die Hälfte dieser Veränderungen zwischen 10-20mm Ausdehnung hatte (Abb. 36). 


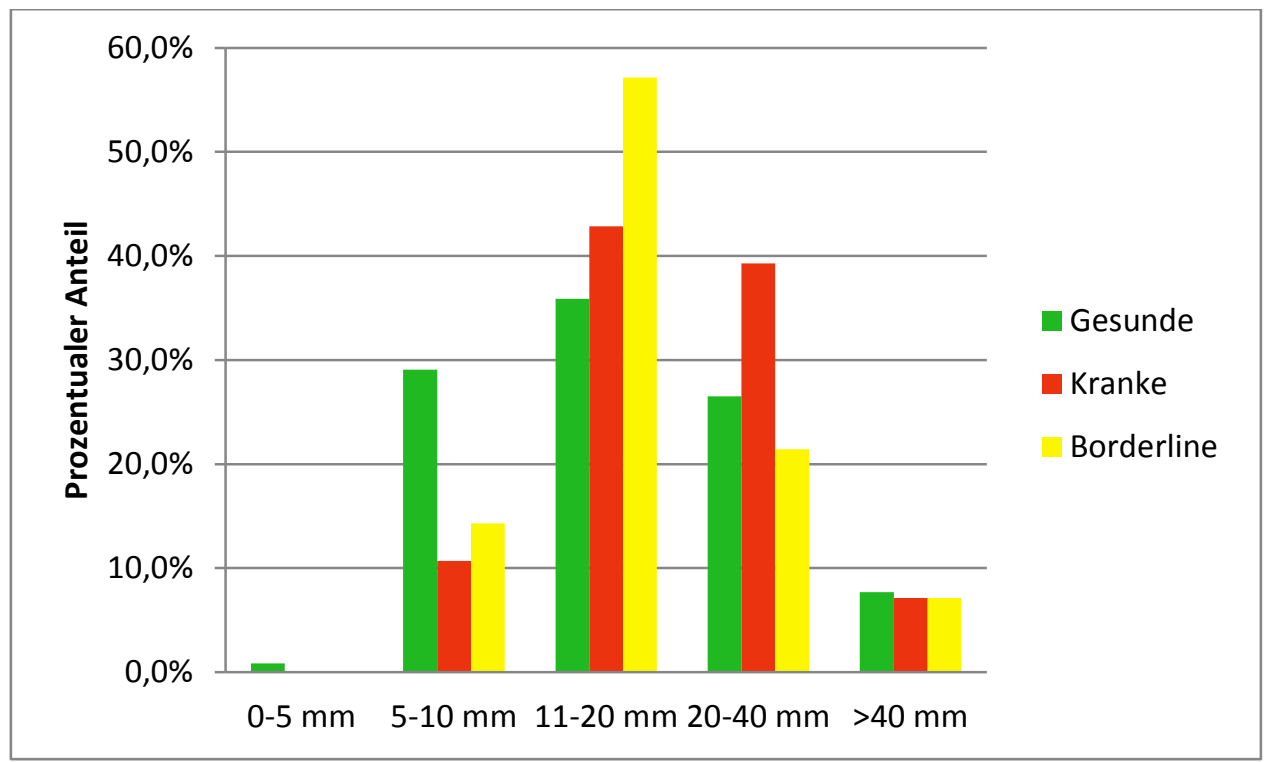

Abbildung 36: Größe der NMLs in Abhängigkeit von der Dignität.

Hinsichtlich der Konfiguration der Kontrastmittelanreicherung der NMLs zeigten die gutartigen Läsionen zu 50\% eine regionale Anreicherungsform. 25\% fielen auf eine lineare und 22,2\% auf eine segmentale Konfiguration. Auch in der Gruppe der bösartigen Veränderungen sowie der Borderline-Läsionen zeigten um die Hälfte der Befunde eine regionale Ausdehnung der NMLs. Je ein Drittel stellte sich hier als linear angeordnet dar (Abb. 37).

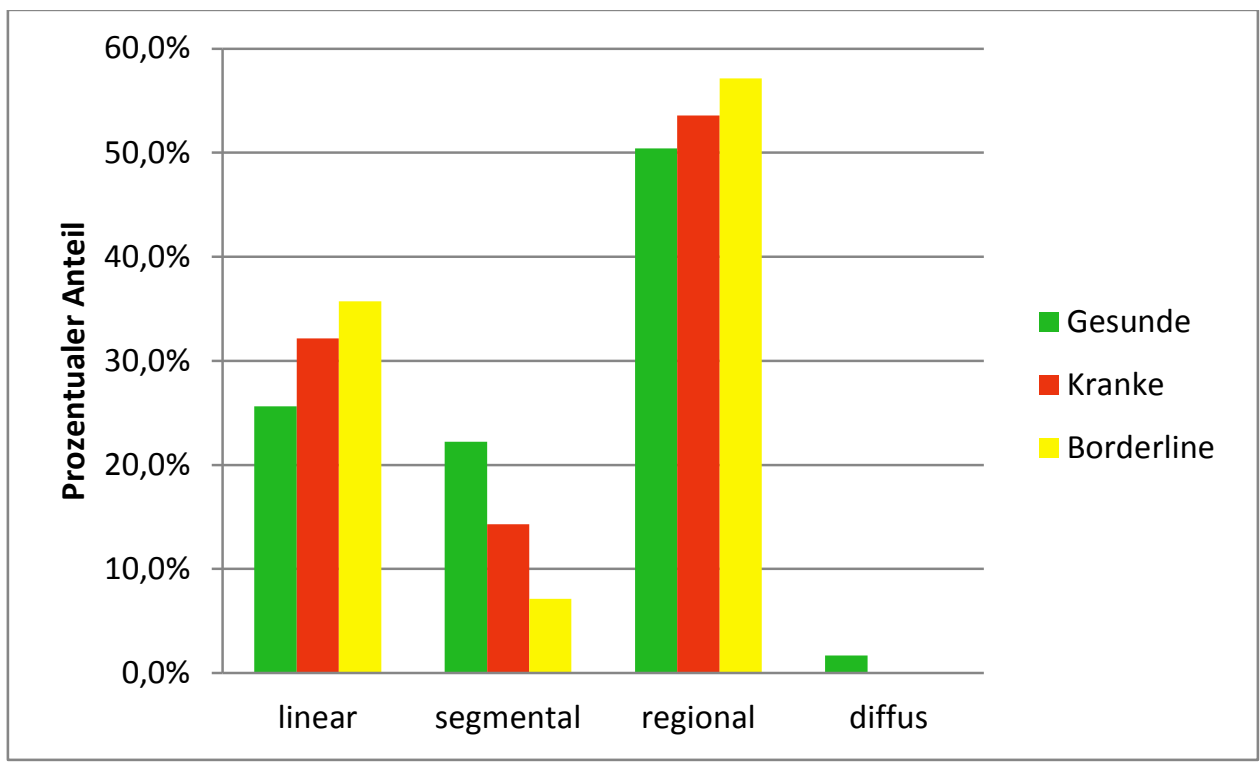

Abbildung 37: Anreicherungsform der NMLs in Abhängigkeit von der Dignität. 


\section{Diskussion}

Die drei etablierten bildgebenden Verfahren Mammographie, Sonographie und MammaMRT nehmen bei der Früherkennung des Mammakarzinoms eine vorrangige Stellung ein. Die Bedeutung der Mammographie und auch die der Mamma-MRT liegt hierbei vor allem in der Detektion von Karzinomen in präinvasiven oder frühen invasiven Stadien. Hierin ist der Grund zu sehen, dass z.B. die Mammographie als primäres Untersuchungsverfahren in nationalen flächendeckenden Früherkennungsprogrammen oder die Mamma-MRT als primäres Untersuchungsverfahren zur Früherkennung von Brustkrebs bei Frauen mit einem Hochrisikoprofil empfohlen wird (Kriege et al. 2004; Schreer et al. 2008; Kuhl et al. 2010).

Im Rahmen der Mammographie-Screening-Programme wird beispielsweise bei asymptomatischen Frauen einer definierten Altersgruppe in bestimmten Intervallen eine Mammographie durchgeführt. Im Falle eines auffälligen Befundes können weitere gezielte Maßnahmen ergriffen werden. Die Aussichten, ein vorhandenes Karzinom zu einem frühen und dadurch prognostisch günstigeren Stadium zu entdecken, werden hierdurch erhöht. Die Hauptaufgabe des Screenings betrifft hierbei weniger die definitive Diagnosestellung, als vielmehr die orientierende Suche nach bildgebenden Auffälligkeiten, die im Rahmen einer gezielten Wiedereinbestellung (sog. „assessment") der entsprechenden Klientinnen abgeklärt werden können. Zielsetzung ist letztendlich eine Verringerung der Brustkrebssterblichkeit (Schulz-Wendtland et al. 2007). Eine Metaanalyse der American Cancer Society aus dem Jahre 2003 belegt eine solche Reduktion der brustkrebsspezifischen Mortalität um 24\% bei adäquatem Screening (Smith et al. 2003). Im Rahmen der Früherkennung des Mammakarzinoms stellt die Mammographie damit das am häufigsten verwendete bildgebende Verfahren dar (Schulz-Wendtland et al. 2007).

Für die Früherkennung des Mammakarzinoms bei Frauen mit 20fach erhöhtem Lebenszeitrisiko für die Entstehung von Brustkrebs (Hochrisiko-Profil) haben bisher vorliegende Studien den hohen Stellenwert der Mamma-MRT belegt. Kriege et al. wiesen eine Sensitivität der MRT für den Nachweis von Brustkrebs von $71 \%$ nach 
(Mammographie 40\%) (Kriege et al. 2004). In der nationalen EVA-Studie konnte in den gemeinsamen Daten von vier deutschen Universitäten ebenfalls gezeigt werden, dass sich die MRT (Sensitivität 93\%) der Röntgenmammographie (Sensitivität 33\%) in diesem Kollektiv als deutlich überlegen erweist (Kuhl et al. 2010). Die limitierte Aussagekraft der Mammographie in diesem Kollektiv erklärt sich im Wesentlichen durch die deutlich reduzierte Aussagekraft des Verfahrens bei Frauen mit dichten (ACR III) oder sehr dichten Parenchymstrukturen (ACR IV), die in dem relativ jungen Untersuchungskollektiv überproportional häufig vorliegen. So betrug das Durchschnittsalter in der EVA-Studie 44,6 Jahre.

Aufgrund der weiten Verbreitung sowie der kostengünstigen Durchführung hat sich auch die Ultraschalluntersuchung der Brust als ein wertvolles diagnostisches Verfahren in der Mammadiagnostik etabliert (Gordon 2002). Vor allem bei einer radiologisch dichten Brust konnten verschiedene Studien zeigen, dass bei 1000 mammographierten Patienten etwa 3-4 Karzinome ausschließlich durch den zusätzlichen Ultraschall diagnostiziert werden (Kolb et al. 1998; Gordon 2002; Kolb et al. 2002). Trotz dieser Mehrinformation gegenüber der Mammographie weist die Ultraschalluntersuchung allerdings Einschränkungen in der Früherkennung des Mammakarzinoms auf. Sie ist insbesondere in der Darstellung intraduktaler Tumorstadien sowie im Nachweis sehr kleiner invasiver Karzinome limitiert (Schulz-Wendtland et al. 2007).

Prinzipiell erfordert die Mammasonographie zudem einen außerordentlich qualifizierten Untersucher. Weitere Limitationen betreffen die große Variabilität des Equipments und die relativ hohe Untersuchungszeit sowie die weiterhin fehlende Standardisierung (Schulz-Wendtland et al. 2007; Order und Schreer 2008). Studien von Kolb et al. sowie Buchberger et al. belegen zudem eine signifikant höhere Rate an falsch positiven Befunden im Vergleich zur Röntgenmammographie (Kolb et al. 1998; Buchberger et al. 1999). Insgesamt wird die alleinige Mammasonographie daher aktuell für die Früherkennung von Brustkrebs als nicht geeignet angesehen (Duda et al. 2008).

Die Auswertung der drei erwähnten bildgebenden Untersuchungsverfahren stützt sich seit mehreren Jahren stark auf die Ausführungen des American College of Radiology, 
die primär für Röntgenmammographie im amerikanischen Screening erarbeitet wurden (American College of Radiology 1993). In der 4. Ausgabe des BIRADS-Lexicons erfolgte eine Auswertung der Ausführungen auch für die Verfahren "Mammasonographie“ und „Mamma-MRT“, die durch die deutsche und die Österreichische Röntgengesellschaft zeitnah deutschsprachig veröffentlicht wurde (American College of Radiology 2004; Fischer U und Helbich 2006).

Das grundsätzliche Verdienst des BIRADS-Lexicons ist zum einen eine einheitliche Terminologie zur Beschreibung der Befundcharakteristika in Mammographie, Ultraschall und Mamma-MRT. Sie beendete damit eine im deutschen Sprachgebrauch lange Zeit sehr individuell gewählte blumenreiche Sprache bezüglich Form, Begrenzung und Binnentextur einer Läsion in der Bildgebung. Zum anderen wurde ein primär 5-, inzwischen 7-stufiges Modell zur Kategorisierung von Befunden in der Bildgebung definiert, das einerseits die Wahrscheinlichkeit für das Vorliegen eines malignen Tumors beschreibt und andererseits Vorgaben für die weitere Vorgehensweise in Abhängigkeit von der jeweiligen Kategorie definiert (American College of Radiology 2004).

Schwachpunkt des BIRADS-Lexicons ist die unbeantwortete Frage, welche Befundkonstellation in der Bildgebung zu welcher konkreten BIRADS-Kategorie führt. Für keines der einzelnen Untersuchungsverfahren gibt es diesbezüglich klare Festlegungen. Letztendlich beinhaltet die Philosophie des Konzeptes, dass in der retrospektiven Auswertung des eigenen Kollektives zu prüfen ist, ob in der jeweiligen Kategorie die erwartete Anzahl an Karzinomen tatsächlich vorgelegen hat. Hieraus kann abgeleitet werden, ob prospektiv eine sachgerechte Befundeinschätzung und Zuordnung zu einer BIRADS-Kategorie erfolgte. Ist dies nicht der Fall, so sollte der Untersucher seine intuitive Zuordnung von Befunden zu einer Kategorie modifizieren.

Für die Zuordnung zu einer bestimmten BIRADS-Kategorie stellt die Schwelle zwischen den Kategorien „BIRADS 3“ und "BIRADS 4“ wohl die schwierigste Entscheidung dar. Einerseits resultiert als Vorgehensweise die Befundkontrolle, die üblicherweise nach einem Zeitraum von 6 Monaten erfolgt, andererseits ist eine Befundabklärung durch 
eine bevorzugt perkutan durchzuführende Biopsie indiziert, die ein invasives Vorgehen impliziert und höhere Kosten verursacht.

Auch die Mamma-MRT erfährt durch das Fehlen klar definierter Kriterien für die Einstufung eines Befundes als benigne oder maligne eine deutliche Limitation, da nicht nur bösartige, sondern auch gutartige Veränderungen mit einer gesteigerten Vaskularisation einhergehen können (Macura et al. 2006). Auch in dem BIRADSLexicon wurde - wie weiter oben aufgeführt - ist nicht festgelegt, wie eine Zuordnung der verschiedenen Befundkriterien zu einer der BIRADS-Kategorien erfolgen sollte. Hier resultierte für das MRT lediglich die Formulierung, dass die Vorgehensweise intuitiv zu erfolgen habe (Zitat: "most approaches are intuitive“) (American College of Radiology 2004, S.99). Zudem wurde an verschiedenen Stellen darauf verwiesen, dass das BIRADS-Lexicon einer Dynamik unterliege und klarere Aussagen in späteren Auflagen zu erwarten seien. Es bleibt abzuwarten, inwieweit in der nächsten Version, die Ende 2011 erwartet wird, diesbezüglich konkrete Vorgehensweisen definiert sein werden.

Aus einer systematischen Überschätzung von Befunden resultiert letztendlich eine reduzierte Spezifität. Für die Mamma-MRT wird in diesem Zusammenhang insbesondere in älteren Studien häufig eine sehr geringe Spezifität der Methode um 49\% und weniger berichtet (Heywang et al. 1989; Gilles et al. 1994; Fischer $U$ et al. 1997; Nunes et al. 1997; Kuhl et al. 1999 b). Ein wesentlicher Grund für diese eher schlechten Ergebnisse ist in der Verwendung unifaktorieller Auswerteprotokolle zu sehen. So ließ z.B. die Arbeitsgruppe um Steven Harms prinzipiell alle MR-Befunde mit einer gesteigerten Kontrastmittelaufnahme operativ abklären. Zusätzliche dynamische oder morphologische Aspekte fanden keine Berücksichtigung, so dass die Spezifität letztendlich erwartungsgemäß nur bei 34\% lag (Harms et al. 1993). Deutlich bessere Ergebnisse für die Spezifität der Mamma-MRT ergaben sich mit der Einführung multimodaler Auswertekonzepte (Fischer U et al. 1994). Unter Berücksichtigung der Signal-Zeit-Kurven sowie kinetischer und morphologischer Kriterien konnte eine Verbesserung der Spezifität in einer Größenordnung um 90\% erreicht werden, ohne gleichzeitig im Bereich der Sensitivität eine Verschlechterung zu erzielen (Schorn et al. 1999; Schnall et al. 2001 ; Baum et al. 2002). 
Der erste Vorschlag zur Ableitung einer konkreten BIRADS-Kategorie auf der Basis der Einzelkriterien erfolgte 1993 in Form eines Punkte-Systems (Göttingen-Score) (Fischer $U$ et al. 1993). Fischer $U$ et al. ordneten den Kriterien eines Befundes in Abhängigkeit von seiner Ausprägung einen Punktwert zwischen 0 und 2 zu. Dieses System erlaubte anhand der Gesamtpunktzahl (0-8) eines Befundes die Zuordnung zu einer BIRADSKategorie (Baum et al. 2002). Die Auswertekriterien im Göttingen-Score umfassten die Form und die Begrenzung sowie die endotumorale Kontrastmittelverteilung, das Ausmaß der initialen Kontrastmittelanflutung und den sog. postinitialen Signalverlauf zwischen der 3. und 8. Minute nach Kontrastmittelgabe (Fischer $U$ et al. 1993). Die Arbeitsgruppe um Fischer konnte für dieses Auswerteprotokoll in einer Studie aus dem Jahre 2002 eine Sensitivität sowie Spezifität für invasive Mammakarzinome von je $92 \%$ nachweisen (Baum et al. 2002).

Es folgten Mitteilungen anderer Arbeitsgruppen, die den Stellenwert des GöttingenScore im eigenen Kollektiv evaluierten. Siegmann et al. untersuchten, ob mit dem Göttingen-Score eine valide BIRADS-Kategorisierung von nur im MRT sichtbaren Mammaläsionen möglich ist. Die Studie an 86 Patienten zeigte für einen GöttingenScore von 4-5 Punkten (BIRADS 4) eine Malignomrate von 24,6\%, bei einem Score von 6-8 Punkten (BIRADS 5) ergaben sich 48,5\% Karzinome. Für einen Göttingen-Score von 3 Punkten (BIRADS 3) konnten keine Karzinome nachgewiesen werden. Die verringerte Anzahl an Karzinomen ist unter dem Gesichtspunkt der niedrigeren Malignomrate von ausschließlich im Mamma-MRT sichtbaren Mammaläsionen gegenüber klinisch, sonographischn und mammographisch detektieren Läsionen zu betrachten. Die Arbeitsgruppe empfiehlt zusammenfassend eine Übertragung des Göttingen-Score in die BIRADS-Klassifikation (Siegmann et al. 2009). Eine weitere Arbeitsgruppe aus dem arabischen Raum fasst zusammen, dass sich die von Fischer beschriebene Evaluationstheorie als sehr nützlich und genau für die Charakterisierung von Mammaläsionen erweist. Bei $92 \%$ der malignen Läsionen konnte in dieser Studie unter Zuhilfenahme der Kombination aus Göttingen-Score und MRM-BIRADS-Lexicon eine korrekte Dignitätsabschätzung erfolgen (Al-Khawari et al. 2009). 
Einigkeit bestand lange Jahre darin, dass neben den morphologischen Kriterien auch dynamische Aspekte wichtig sind. So kommen Wedegärtner et al. in ihrer Studie aus dem Jahre 2001 zu dem Schluss, dass die Aussagekraft der morphologischen Kriterien geeignet ist um zwischen gut- und bösartigen Befunden zu unterscheiden, jedoch ergänzend eine Beurteilung der dynamische Parameter erfolgen sollte (Wedegärtner et al. 2001). Die Empfehlung, neben morphologischen auch dynamische Kriterien zur Beurteilung heranzuziehen, wird auch von den Arbeitsgruppen von Kuhl et al., Schnall et al. und Warren et al. gegeben (Kuhl et al. 1999 b; Schnall et al. 2006; Warren et al. 2006). Neue Arbeiten verweisen inzwischen allerdings mehr und mehr darauf, dass durch eine Verbesserung der räumlichen Auflösung der Mamma-MRT mit Verwendung einer nicht interpolierten Bildmatrix von 512x512 oder höher den morphologischen Kriterien eine immer höhere Bedeutung zukommt, während die Signalkurven-Analyse zunehmend in den Hintergrund tritt (Kuhl et al. 2005; Fischer U 2010).

Unabhängig von der Diskussion um die Wertigkeit dynamischer Kriterien hinterfragten verschiedene Arbeitsgruppen neben der Nützlichkeit des Göttingen-Score auch die Effektivität zusätzlicher Auswertekriterien wie u.a. Signalintensität in der wassersensitiven Messung, die Volumenzunahme einer anreichernden Läsion im zeitlichen Verlauf („blooming“) sowie den Nachweis minderdurchbluteter endotumoraler Septen (Fischer U et al. 2005; Malich et al. 2005; Kuhl 2007; Siegmann et al. 2009). So konnten Siegmann et al. in einer Studie ein signifikantes Korrelat zwischen Malignomrisiko und der nach dem Göttingen-Score zugeordneten BIRADS-Kategorie feststellen. Aufgrund einer Malignomrate von lediglich 18,2 \% bei einer MRM-BIRADSKategorie 4 gemäß Göttingen-Score schlagen die Autoren jedoch vor, bei einer solchen Läsion eine Verlaufskontrolle anstelle der Biopsie durchzuführen, sofern die Größe des Befundes $<10 \mathrm{~mm}$ ist. Hierdurch würde die Rate falsch positiver Biopsien verringert (Siegmann et al. 2009). Bezüglich dieses Vorschlages sei jedoch kritisch angemerkt, dass gerade beim Verzicht auf die Abklärung kleiner Befunde für die entsprechenden Patienten die Chance auf eine Früherkennung, d.h. Erkennung von Tumoren in einem prognoserelevanten Studium, vertan wird. 
Ein anderes Auswerteprotokoll stellten Malich et al. 2005 vor. Es beruht ebenfalls auf einer Kombination von morphologischen und dynamischen Kriterien. Zusätzlich wurden zur Verbesserung der Genauigkeit und Zuverlässigkeit der MRM-Bildgebung weitere Evaluationskriterien hinzugefügt: Signalintensität in der T2-Messung, allmähliche zunehmende Unschärfe der Begrenzung im zeitlichen Verlauf (blooming), die Vergrößerung axillärer Lymphknoten auf $>10 \mathrm{~mm}$, das sog. "hook sign“ als Korrelat einer Infiltration des Pektoralismuskels, minderdurchblutete endotumorale Septierungen, eine Unterbrechung der Mamillen-Linie, Ödeme, eine potentielle Hautverdickung sowie ein auf eine Läsion zuführendes Gefäß (Malich et al. 2005). Kritisch sei hier angemerkt, dass ein großer Teil dieser zusätzlich gewählten Auswertekriterien sekundäre Kriterien eines großen bzw. fortgeschrittenen Tumorgeschehens darstellen und für kleine, prognostisch günstige Tumoren wohl nicht zutreffen dürften. Es muss somit offen bleiben, inwieweit diesen Kriterien in der Frühdiagnostik des Mammakarzinoms überhaupt eine Bedeutung zukommen kann.

Fischer DR et al. legten im Jahre 2005 eine retrospektive Studie vor, in der die Aussagekraft des Göttingen-Score in Kombination mit dem von Malich et al. entwickeltem System evaluiert wurde. Sie kamen in dieser Studie für die alleinige Anwendung des Göttingen-Score auf eine Sensitivität von 83,1\% und eine Spezifität von $58,8 \%$. Für eine Kombination der beiden Evaluationsstrategien konnte eine Verbesserung der Sensitivität auf $97 \%$ und der Spezifität auf 76,5\% nachgewiesen werden (Fischer DR et al. 2005). Allerdings lag die durchschnittliche Tumorgröße in diesem Kollektiv bei $24 \mathrm{~mm}$. Es bleibt zudem fraglich, ob eine Erweiterung des Göttingen-Score um die von Malich et al. geforderten Merkmale für die Routineanwendung als sinnvoll zu erachten ist. Die 13 Merkmalausprägungen des Göttingen-Score müssten um die 18 aus der von Malich vorgestellten Strategie erweitert werden. Für den Alltag kommentieren Siegmann et al. ein solches modifiziertes Konzept daher als sehr komplex und kaum realisierbar (Siegmann et al. 2009). Gegenwärtig gipfelt die Anzahl an Auswertekriterien in einer Monographie von W.A. Kaiser, in der 100 Zeichen in der Mamma-MRT beschrieben werden (Kaiser 2007). Unbeantwortet bleibt hierbei allerdings ebenso die Frage, in welcher Weise die Ausprägung eines jeden 
Einzelkriteriums schlussendlich in eine Gesamtkategorisierung des Befundes führen soll.

Für die Mammographie wird von verschiedenen Autoren beschrieben, dass der Anteil an Befunden, die als BIRADS 3 kategorisiert wurden, zwischen 3 bis $11 \%$ beträgt (Sickles 1991; Varas et al. 1992; Vizcaino et al. 2001; Varas et al. 2002). Liberman et al. charakterisierten im Jahre 2003 in der Mamma-MRT 24\% einer Studienpopulation als "wahrscheinlich benigne" (Liberman et al. 2003 a). Diese hohe Rate an BIRADS 3 Befunden ist wahrscheinlich auf das geringe Alter der Studienpopulation zurückzuführen. Über die Hälfte der Patienten war prämenopausal, so dass gehäuft hormonell induzierte Veränderungen im Zusammenhang mit dem Menstruationszyklus zu einem Befund mit verstärkter Kontrastmittelanreicherung führten (Kuhl et al. 1997). Der Anteil von 6,2\% am Gesamtkollektiv für die Kategorie MRM-BIRADS 3 in dieser Studie korreliert mit den Ergebnissen von Sickles, Varas et al. sowie Vizcaino et al. (Varas et al. 1992; Sickles 1995; Vizcaino et al. 2001). Mit 7,8\% stellten sich die BIRADS-4-Befunde allerdings seltener dar als in bisher publizierten Studien von Orel et al. (71\%) sowie Liberman et al. (72\%) (Liberman et al. 1998; Orel et al. 1999). Diese vermeintliche Diskrepanz ist darin begründet, dass Orel und Liberman symptomatische Patienten untersuchten, während im eigenen Kollektiv viele Frauen im Rahmen der Früherkennung kamen, also asymptomatisch waren. Zudem spielt eine Rolle, ob es sich bei der Untersuchung um ein Erst- oder ein Folge-MRT handelt. So berichten Abramovici und Mainiero für den Einsatz der Mamma-MRT im Screening über eine Quote an BIRADS 3 Einschätzungen von 10,1\% (BIRADS 4/5 mit 5,9\%) bei Erst-MRT und Rückgang dieser Quoten auf 2,6\% (BIRADS 3) bzw. 4,7\% (BIRADS 4), sofern eine Voruntersuchung vorlag (Abramovici und Mainiero 2011).

Die eigenen Untersuchungen belegten, dass sich bezogen auf die Entitäten eine Gleichverteilung in den Kategorien BIRADS 3 und 4 zeigte. Weder Foki, NMLs noch Herde stellten sich auffallend häufig in einer der beiden BIRADS-Kategorie dar, was somit auch keine abschließende Einschätzung der Dignität des Befundes zuließ. 
Für die Foki zeigte sich in der vorliegenden Studie der Trend, dass Befunde, die einen erhöhten Wassergehalt gegenüber dem angrenzenden Mammagewebe aufwiesen, vornehmlich für eine Einordnung in die Kategorie 3 sprachen, während Befunde mit erniedrigten endotumoralen Flüssigkeitsgehalt eher der Kategorie BIRADS $4 \mathrm{zu}$ zuordnen waren. Diese Tendenz beschrieben bereits Kuhl et al. in einer Studie aus dem Jahre 1999 (Kuhl et al. 1999 a). In einer retrospektiven Studie untersuchten Liberman et al. 666 nicht tastbare nur im MRM identifizierbare Läsionen. Sie stellten fest, dass die Wahrscheinlichkeit für das Vorliegen eines Karzinoms signifikant mit der Läsionsgröße zusammenhängt. Lediglich $3 \%$ der insgesamt 37 Läsionen kleiner als $5 \mathrm{~mm}$ erwiesen sich als bösartig (Liberman et al. 2006).

Eby et al. reevaluierten 2009263 als BIRADS 3 kategorisierte Läsionen. Lediglich 0,85\% stellten sich als maligne heraus, was auf der einen Seite die Sicherheit der Kategorie BIRADS 3 als Alternative zur Biopsie bestätigt, auf der anderen Seite aber auch aufzeigt, dass eine große Anzahl gutartiger Befunde zu der wahrscheinlich benignen Kategorie zuordnet wurde. Die wiederum hieraus resultierende kurzfristige Verlaufskontrolle ist mit einem höheren Kostenaufwand sowie einer gesteigerten psychischen Belastung für die Patienten verbunden. Die Arbeitsgruppe kam zu dem Schluss, dass Foki aus der BIRADS 3 Gruppe, die einen kontinuierlichen postinitialen Signalverlauf aufweisen, der Kategorie BIRADS 2 zugeordnet werden können, ohne dabei in der Kategorie 3 die von dem ACR geforderte Krebsrate von weniger als $2 \%$ zu überschreiten (Eby et al. 2009). Dies ist allerdings nicht so zu verstehen, dass nach Klassifizierung einer Läsion als Fokus, diese per se als benigne einzuschätzen ist. Lediglich Biopsien sind in dieser Kategorie selten notwendig, da die Wahrscheinlichkeit eines malignen Wachstums sehr gering ist. Mit dieser Empfehlung decken sich auch die Ergebnisse der vorgelegten Studie. In der Gruppe der Foki gab es letztendlich und unabhängig von der BIRADS-Kategorie lediglich ein Mammakarzinom (Größe 4mm). Dies entspricht einer Quote von $<0,3 \%$.

Prinzipiell wiesen über $80 \%$ der Herde eine Größenordnung zwischen 5-10mm auf. Dies unterstreicht die vorrangige Stellung der Mamma-MRT bei der Früherkennung. Läsionen dieser Größenordnung sind klinisch oder mittels anderer bildgebender 
Verfahren oft okkult. Für Herdbefunde ab $1 \mathrm{~cm}$ Größe steigt erwartungsgemäß die Aussagekraft der Sonographie, so dass solche Befunde oft primär im Ultraschall oder aber im sonographischen „Second Look" detektiert werden können.

Verschiedene Arbeitsgruppen prüften den Zusammenhang zwischen Läsionsgröße und Malignität. In einer retrospektiven Studie untersuchten Liberman et al. 666 nicht tastbare, nur in der MRT identifizierbare Läsionen. Sie fanden heraus, dass die Wahrscheinlichkeit für das Vorliegen eines Karzinoms signifikant mit der Läsionsgröße zusammenhängt. Lediglich $3 \%$ der insgesamt 37 Läsionen kleiner als $5 \mathrm{~mm}$ erwiesen sich als bösartig (Liberman et al. 2006). Auch bei Siegmann et al. hängt das Malignomrisiko signifikant mit zunehmender Läsionsgröße zusammen. Nach Meinung der Autoren sollte daher bei kleineren Läsionen $(<10 \mathrm{~mm})$ der Kategorie BIRADS 4 eine Verlaufskontrolle anstelle einer Biopsie erwogen werden (Siegmann et al. 2009). Dies ist jedoch auch kritisch zu sehen, da sich eine Kontrolle dieser Befunde aufgrund der geringen Größe mittels Ultraschall oder Mammographie als schwierig gestalten kann. Erst bei einer Größe von $10 \mathrm{~mm}$ können im Screening mittels Ultraschall Malignome zuverlässig nachgewiesen werden (Davis und McCarty 1997).

Unter den Score-Einzelkriterien der Herde erwies sich das Merkmal der unscharfen äußeren Kontur als Parameter mit der besten Differenzierungsmöglichkeit zwischen malignen und benignen Befunden. Auch wenn viele Publikationen die irregulären Form als spezifischstes Merkmal für Malignität beschreiben (Gilles et al. 1994; Wedegärtner et al. 2001; Liberman et al. 2003 b), konnte in der vorliegenden Studie diesem Parameter keine Bedeutung für die Dignitätsabschätzung nachgewiesen werden. Lediglich $26 \%$ maligne Herde wiesen eine irreguläre Form auf. Die Ursache hierfür ist mit großer Wahrscheinlichkeit in der Interobserver-Variabilität zu finden. Wahrscheinlich werden runde Befunde aufgrund ihrer unscharfen Begrenzung bevorzugt als irregulär oder gar spikuliert beschrieben, wenngleich dies das Kriterium der Begrenzung und nicht der Form darstellt.

Des Weiteren ließ auch die Größe der Läsion keine weitere Charakterisierung bezogen auf die BIRADS-Kategorien zu. Veranschaulicht man sich die Begrenzung der Herde in 
dieser Studie, so war zu erkennen, dass Befunde mit unscharfer Begrenzung gehäuft in die Kategorie BIRADS 4 eingeordnet wurden, Läsionen mit glatter Begrenzung entsprachen eher einem Befund der Kategorie BIRADS 3.

Bezogen auf das endotumorale Verteilungsmuster weisen verschiedene Studien darauf hin, dass ein randständiges Anreicherungsmuster stark auf Malignität hinweist (Ikeda et al. 2001; Wedegärtner et al. 2001; Schnall et al. 2006). Nunes et al. beschrieben einen positiven prädiktiven Wert für Malignität von $79-92 \%$ beim Vorliegen eines Ringenhancements (Nunes et al. 1997). Diese Aussagen decken sich mit den Ergebnissen der vorliegenden Studie. Bei mehr als einem Drittel der bösartigen Befunde fand sich ein Ringenhancement, bei gutartigen Läsionen waren es deutlich weniger.

In der vorliegenden Arbeit zeigten sämtliche Herdbefunde einen initialen Signalanstieg von über $50 \%$, somit konnten keine langsam anreichernden Tumore festgestellt werden. Dies steht auch im Einklang mit den Ergebnissen von Liberman et al., auch sie kamen zu dem Schluss, dass die Frequenz der im Verlauf malignen Befunde am niedrigsten bei Läsionen mit einem kontinuierlichen kinetischen Signalverlauf war (Liberman et al. 2003 a). Lediglich Heywang-Köbrunner berichtete über eine vergleichsweise hohe Rate an langsam anreichernden, im Verlauf stetig ansteigenden Karzinomen. Grund hierfür ist jedoch eine zeitlich sehr schlecht auflösende Messtechnik mit nur zwei Serien nach Kontrastmittelgabe und einer Meßzeit pro Schichtstapel um 5 Minuten (HeywangKobrunner 1994). Solche Techniken mit geringer zeitlicher Auflösung kommen heute nicht mehr zum Einsatz.

Für den postinitialen Signalverlauf konnte in der vorliegenden Arbeit gezeigt werden, dass keine der malignen Läsionen einen kontinuierlichen Signalanstieg nach Kontrastmittelgabe aufwies. Hier fand sich in je fast $50 \%$ der Fälle ein Plateau- oder ein Auswaschphänomen. Dies deckt sich mit den Untersuchungen von Kuhl et al. zum postinitialen Signalverlauf. Nach den Angaben dieser Studie ist das Vorliegen eines wash out Phänomens mit einer Brustkrebswahrscheinlichkeit von $87 \%$ verbunden und ist laut Meinung der Autoren eine klare Indikation zur bioptischen Abklärung des Befundes (Kuhl et al. 1999 b). 
Bei der Vergegenwärtigung des Wassergehalts der Herdbefunde in der vorliegenden Studie wurde der Trend deutlich, dass Befunde die einen erhöhten Wassergehalt gegenüber dem angrenzenden Mammagewebe aufwiesen, vornehmlich gutartig waren, während Befunde mit erniedrigten endotumoralen Flüssigkeitsgehalt für Malignität sprachen. Eine Schnittmenge stellt ein isotenses Signal dar, hier war es an Hand der vorliegenden Daten nicht möglich, zwischen maligne und benigne zu unterscheiden. Dies steht im Einklang mit den Ergebnissen von Kuhl et al. die bei der Untersuchung von 205 malignen und benignen Brusttumoren ermittelten, dass maligne Befunde zu 87\% ein iso- oder hypointenses Signal aufwiesen, benigne Befunde hingegen stellten sich zu $71 \%$ als hyperintens dar (Kuhl et al. 1999 a).

Die präsentierten Studienergebnisse zeigten, dass sich für die Punktwerte des Göttingen-Score der Herdbefunde eine diskrete Trennmarge für die einzelnen Dignitäten abzeichnete. Benigne Befunde erzielten in den meisten Fällen Scorewerte von 3 bis 5 , während bei den malignen Befunden hauptsächlich Punktwerte von 4 bis 6 erreicht wurden. Somit konnte konstatiert werden, dass Scorewerte von 3 eher für benignes Wachstum sprechen, während Werte von 6 auf maligne Läsionen hinwiesen. GöttingenScorewerte von 4 und 5 ließ keine weitere Dignitätsabschätzung zu. Dies deckt sich mit den Angaben von Siegmann et al. in einer Studie aus dem Jahre 2009. Sie ermittelten für einen Göttingen-Score von 3 Punkten eine Malignomrate von $0 \%$. Bei einem Punktwert von 6 betrug die Rate bösartiger Tumoren 50\% (Siegmann et al. 2009).

Für die NMLs ließ sich keine Dominanz einer einzelnen Kategorie bezogen auf die Größe oder Konfiguration nachweisen. In beiden Kategorien stellten sich die Läsionen hauptsächlich mit einer Größe von 5-40mm dar. Grund für die relativ hohe Größe der Läsionen sind die Tumorentitäten, die typischerweise als NML imponieren. Dies sind im gutartigen Bereich Adenosen und Fibrosen und im Bereich der bösartigen Tumoren das DCIS und diffus wachsende invasiv lobuläre Mammakarzinome (Fischer U 2010).

Die Anzahl an falsch negativen Befunden in der Kategorie MRM-BIRADS 3 betrug in dieser Studie 3,1\%. Dieser Wert ist gegenüber der von dem ACR in der 4. Auflage (American Collage of Radiology 2004) geforderten Malignomrate von $<2 \%$ (3. Auflage: 
$<3 \%$ ) (American Collage of Radiology 1993) für die Befunde der Kategorie BIRADS 3 leicht erhöht. Allerdings berichteten auch frühere Untersuchungen von einem breiten Spektrum der Malignomrate (0,6-10\%) unter den wahrscheinlich benignen Befunden (Kuhl et al. 2000; Liberman et al. 2003 a; Kriege et al. 2004; Sadowski und Kelcz 2005; Eby et al. 2009). Die in der vorliegenden Studie leicht erhöhte Malignomrate muss auch vor dem Hintergrund der vergleichsweise geringen Anzahl an BIRADS-3-Befunden ( $n=196)$ gesehen und darf nicht überbewertet werden. Eby et al. wiesen bei einem sehr viel größeren Kollektiv ( $n=2569$ ) bei lediglich 0,85\% der BIRADS-3-Befunde Malignität nach (Eby et al. 2009).

Die suspekten Befunde der Kategorie BIRADS 4 weisen laut ACR eine Karzinomwahrscheinlichkeit von 2-90\% auf. Orel et al. untersuchten anno 1997 eine Anzahl von 1312 mammographisch nachgewiesenen Läsionen, welche im späteren Verlauf offen biopsiert wurden und kategorisierten diese unter zu Hilfenahme des BIRADS-Lexicons. 936 (71\%) der Läsionen wurden der Kategorie BIRADS 4 zugewiesen. Letztlich stellten sich $30 \%$ der Läsionen als maligne heraus (Orel et al. 1999). Diese Werte sind fast identisch mit den Resultaten einer Studie von Liberman et al.. Sie untersuchten 492 Läsionen, auch hier wurden $72 \%$ der untersuchten Befunde der Kategorie 4 zugewiesen, die Malignomrate lag in diesem Kollektiv bei $31 \%$.

Auch in der vorliegenden Arbeit stellte sich etwa ein Drittel $(34,8 \%)$ der Läsionen in der Kategorie BIRADS 4 als maligne dar. Diese Ergebnisse sind somit vergleichbar mit den publizierten Daten von Liberman et al. und Orel et al. an einem ähnlichen Patientengut, auch wenn eine andere Bildgebung (Mammographie vs. Mamma-MRT) zum Einsatz kam.

Mit Blick auf die BIRADS-Kategorisierung haben sich die verschiedenen Arbeitsgruppen für alle drei etablierten Untersuchungsverfahren schwerpunktmäßig mit den Klassifikationen BIRADS 3 und BIRADS 4 beschäftigt, da an dieser Stelle die Schwelle von konservativer Vorgehensweise zu invasiver Befundabklärung überschritten wird. 
Für wahrscheinlich benigne Befunde (Kategorie BIRADS 3) wird bei einer Karzinomwahrscheinlichkeit von unter $2 \%$ eine kurzfristige Verlaufskontrolle der Befunde empfohlen (American College of Radiology 2004). Begründet wird diese Vorgehensweise damit, dass maligne Befunde anhand der Größenzunahme in der Kontrolluntersuchung nach 6 Monaten noch ausreichend früh diagnostiziert werden können und somit noch nicht mit einer schlechteren Prognose einhergehen (Varas et al. 1992; Sickles 1995). Im eigenen Kollektiv erschienen annähernd 90\% der Frauen mit einem Befund der Kategorie MRM-BIRADS 3 zur empfohlenen Kontrolluntersuchung. Dies entspricht einer guten Compliance.

Sickles erstellte für die Mammographie ein Follow-up-Protokoll, nach welchem Patienten mit Befunden der Kategorie BIRADS 3 eine Mammographie nach 6, 12, 24 und 36 Monaten erhielten. Unter diesen Voraussetzungen konnten 67\% der malignen Läsionen, die ursprünglich als BIRADS 3 kategorisieren wurden, nach 6 Monaten diagnostiziert werden. Nach 6 weiteren Monaten waren 94\% der Malignome identifiziert (Sickles 1991). Angemerkt werden muss allerdings, dass dieses Kollektiv einen hohen Anteil an Mikroverkalkungen aufwies und daher von einer Frühform des Mammakarzinoms mit zum Teil sehr geringen Proliferationsraten auszugehen ist. Für Herdbefunde zwischen 5 und $10 \mathrm{~mm}$ sowie NMLs in der in dieser Studie ausgeführten Größenordnung dürfte die Diagnosestellung in aller Regel nach einem Intervall von 6 Monaten gelingen. Die von Sickles angegebenen Kontroll-Intervalle werden laut ACR auch für BIRADS-3-Befunde in der Sonographie und Mamma-MRT empfohlen (American College of Radiology 2004).

Ein Befund der Kategorie BIRADS 4 bedarf laut ACR einer histologischen Abklärung (American College of Radiology 2004). Dies kann nach nationaler S3-Leitlinie mit einem perkutan-bioptischen Verfahren oder einer offenen Biopsie geschehen (Lebeau et al. 2008). Ein großes Problem bei der histologischen Abklärung dieser unklaren Befunde ist der psychische und physische Stress, den die Patienten im Laufe einer entsprechenden Intervention unterliegen. Darüber hinaus stellt sich natürlich die Kostenfrage, da sowohl perkutan-bioptische Verfahren - hier insbesondere die Vakuumbiopsie - und offene Biopsien kostentreibend sind. Prinzipiell sollte empfohlener Maßen angestrebt werden, 
unklare Befunde der Mamma perkutan-bioptisch abzuklären, sofern in begründeten Einzelfällen nicht eine offene Biopsie indiziert ist (Kettritz et al. 2004).

In Abhängigkeit von der zu klärenden Läsion und der Bildgebung kann die perkutane Biopsie sonographisch, mammographisch oder MRT-gestützt durchgeführt werden. Für entsprechende Befunde in der MRT, die in Mammographie und Ultraschall kein eindeutiges Korrelat aufweisen, wird die perkutan-bioptische Gewebeentnahme durch eine Vakuumbiopsie empfohlen. Die Arbeitsgemeinschaft Mammadiagnostik der Deutschen Röntgengesellschaft hat hierfür qualitätssichernde Rahmenbedingungen formuliert (Bick et al. 2008).

In der präsentierten Studie wurden alle histologisch abzuklärenden Gewebeproben der Kategorie BIRADS 4 leitliniengemäß vakuumbioptisch entnommen. Der Anteil der benignen und der malignen Befunde sowie der Borderline-Läsionen betrug 54,1\%, 32,3\% bzw. 13,6\%. Dies steht im Einklang mit den Ergebnissen einer Multicenter Studie aus dem Jahre 2006, in der die Ergebnisse der MR-Vakuumbiopsie bei 538 Patienten präsentiert wurden: Die Quote gutartiger Befunde betrug hier $70 \%$ und die Rate bösartiger Prozesse 27\%. 3\% der Befunde waren Borderline-Läsionen (Perlet et al. 2006). Auch bei Liberman et al., Orel et al sowie in einer früheren Auswertung von Fischer $U$ et al. zeigte sich nach durchgeführter Vakuumbiopsie ein ähnliches Verteilungsmuster der einzelnen Dignitäten (Liberman et al. 1998; Orel et al. 1999; Fischer $U$ et al. 2009). Letztendlich decken sich die präsentierten Quoten auch mit denen mammographisch gesteuerter Vakuumbiopsien bei Mikrokalk (sog. stereotaktische Vakuumbiopsie). In einer Multicenter-Studie wurden stereotaktische Vakuumbiopsie bei 2874 Patienten ausgewertet. Die Quote an gutartigen und bösartiger Tumoren sowie Borderline-Läsionen betrug hierbei 73\%, 22\% und 5\% (Kettritz et al. 2004).

Diese bisher vorliegenden Daten zur MR-gesteuerten Vakuumbiopsie zeigen, dass diese Intervention zur definitiven Abklärung von Befunden der Kategorie MRM-BIRADS 4 etabliert ist. Die prätherapeutische Sicherung der Histologie erlaubt bei bösartiger Histologie eine adäquate Planung der Behandlungsstrategie, z.B. zur Frage eines 
neoadjuvanten Konzeptes, der konkreten operativen Vorgehensweise oder der Strategie für die Lymphadenektomie. Im Falle einer gutartigen Histologie kann auf einen unnötigen operativen Eingriff verzichtet werden und eine konsequente Kontrolle nach 6 Monaten erfolgen. Bei Befunden mit unklarem biologischem Potential sollte die weitere Vorgehensweise in einer interdisziplinären Runde festgelegt werden. 


\section{Zusammenfassung}

Im Zeitraum von 06/2003 bis 06/2009 erfolgten im Diagnostischen Brustzentrum Göttingen bei 5182 Frauen diagnostische Mamma-MRT-Untersuchungen. Hiervon wurden 6,2\% ( $n=321)$ als Befunde der Kategorie MRM-BIRADS 3 und 7,8\% ( $n=405)$ als Befunde der Kategorie MRM-BIRADS 4 bewertet. Innerhalb der präsentierten Studie wurden aus diesen beiden Gruppen die Befunde von 394 Patienten (MRM-BIRADS 3: 196 Patienten; MRM-BIRADS 4: 198 Patienten) evaluiert.

Die Häufigkeit an Foki (10\%), Herdbefunden (46\%) und nicht-raumfordernden Läsionen [NML] (43\%) war in beiden Kategorien (MRM-BIRADS 3 und MRM-BIRADS 4) etwa gleich hoch.

In der Gruppe der Foki $(<5 \mathrm{~mm}$ ) lag die Quote an Karzinomen <3\% (1 Karzinom auf 42 Foki). Prinzipiell erscheint daher eine Kategorisierung unklarer Foki als MRM-BIRADS 2 mit konsequenter Überwachung im Rahmen der Früherkennung (Intervall 1 Jahr) sinnvoll.

In der Gruppe der Herdbefunde, deren Größe zwischen 5-10mm dominierte, ließen sich keine Befundmuster ableiten, die eine zuverlässige Zuordnung zu den Kategorien MRMBIRADS 3 oder MRM-BIRADS 4 ermöglichten, da für alle Kriterien Überlappungsbereiche vorlagen. Die deutlichsten Kriterien für Malignität waren die unscharfe Begrenzung, das Auswaschphänomen und der niedriger endotumorale Wassergehalt.

Der Göttingen-Score, der insbesondere zur Einschätzung von Herdbefunden weiterhin sinnvoll erscheint, erlaubt bis zu einer Gesamtzahl von 3 Punkten ein abwartendes Verhalten ohne histologische Abklärung (Quote an Karzinomen: 3,6\%). Bei einer Gesamtzahl von 6 Punkten oder mehr sollte ausdrücklich eine perkutan-bioptische Abklärung erfolgen (Quote an Karzinomen: 69,2\%). Diese Befunde können vorzugsweise als MRM-BIRADS 4C (Karzinomwahrscheinlichkeit 60-90\%) verschlüsselt werden. Bei einer Anzahl von 4 oder 5 Punkten liegen die Wahrscheinlichkeiten für das 
Vorliegen eines Karzinoms bei $19,2 \%$ bzw. $26,2 \%$, so dass ein Herdbefund mit einem Score von 4 Punkten eine Verschlüsselung als BIRADS 4A (Karzinomwahrscheinlichkeit 2-30\%) und ein Herdbefund mit 5 Punkten eine Kategorisierung als MRM-BIRADS 4B (Karzinomwahrscheinlichkeit 30-60\%) nahelegen.

Für NML zeichnet sich überhaupt keine Möglichkeit einer Differenzierung zwischen gutund bösartigen Prozessen bzw. einer Zuordnung zu einer der beiden BIRADS-Gruppen $a b$, da hier für alle Kriterien große Überlappungsbereiche resultieren. 


\section{Literaturverzeichnis}

Abramovici G, Mainiero MB (2011): Screening breast MR imaging: comparison of interpretation of baseline and annual follow-up studies. Radiology 259 , 85-91

ACR (American College of Radiology) (1993): Breast imaging reporting and data system (BI-RADS). Reston VA: American College of Radiology

ACR (American College of Radiology) (2004): BI-RADS - magnetic resonance imaging. in: ACR breast imaging reporting and data system, breast imaging atlas. Reston VA: American College of Radiology

Albert U, Naß-Griegoleit I: Gesundheitsverhalten: Stellenwert der BrustSelbstuntersuchung; in: Stufe-3-Leitlinie Brustkrebs-Früherkennung in Deutschland; hrsg. v. Albert U; Zuckschwerdt, München 2008, 68-72

Al-Khawari H, Athyal R, Kovacs A, Al-Saleh M, Madda JP (2009): Accuracy of the Fischer scoring system and the Breast Imaging Reporting and Data System in identification of malignant breast lesions. Hematol Oncol Stem Cell Ther $\underline{2}$, 403410

Allred DC, Mohsin SK (2000): Biological Features of Premalignant Disease in the Human Breast. J Mammary Gland Biol Neoplasia $\underline{5}$, 351-364

Barleon B, Siemeister G, Martiny-Baron G, Weindel K, Herzog C, Marme D (1997): Vascular endothelial growth factor up-regulates its receptor fms-like tyrosine kinase 1 (FLT-1) and a soluble variant of FLT-1 in human vascular endothelial cells. Cancer Res $\underline{57}, 5421-5425$

Baum F, Fischer U, Vosshenrich R, Grabbe E (2002): Classification of hypervascularized lesions in CE MR imaging of the breast. Eur Radiol 12, 10871092

Becker N (2001): Entwicklung der Inzidenz und Mortalität an Brustkrebs. Radiologe $\underline{41}$, 337-343

Beckmann MW, Niederacher D, Goecke T, Bodden-Heidrich R, Schnürch H, Bender H (1997): Hochrisikofamilien mit Mamma- und Ovarialkarzinomen. Dtsch Arztebl Int 94, 161-167

Berg WA, Gutierrez L, NessAiver MS, Carter WB, Bhargavan M, Lewis RS, loffe OB (2004): Diagnostic accuracy of mammography, clinical examination, US, and MR imaging in preoperative assessment of breast cancer. Radiology $\underline{233}, 830-849$

Bick U, Fischer U, Kuhl C, Mülelr-Schimpfle M, Schulz-Wendtland R: Interventionelle Techniken; in: Stufe-3-Leitlinie Brustkrebs-Früherkennung in Deutschland; hrsg. v. Albert US; Zuckschwerdt, München 2008, 140-145 
Böcker W, Denk H, Heitz P: Pathologie, 2. Auflage; Urban\&Fischer, München 2001

Boyd NF, Guo H, Martin LJ, Sun L, Stone J, Fishell E, Jong RA, Hislop G, Chiarelli A, Minkin S, Yaffe MJ (2007): Mammographic density and the risk and detection of breast cancer. N Engl J Med $\underline{356}, 227-236$

Brinck U: Tumorangiogenese; in: Atals der MR-Mammograohie;hrsg. v. Fischer U; Thieme, Suttgart 2000, 22-23

Buadu LD, Murakami J, Murayama S, Hashiguchi N, Sakai S, Toyoshima S, Masuda K, Kuroki S, Ohno S (1997): Patterns of peripheral enhancement in breast masses: correlation of findings on contrast medium enhanced MRI with histologic features and tumor angiogenesis. J Comput Assist Tomogr 21, 421-430

Buchberger W, DeKoekkoek-Doll P, Springer P, Obrist P, Dunser M (1999): Incidental findings on sonography of the breast: clinical significance and diagnostic workup. AJR Am J Roentgenol $\underline{173}, 921-927$

Claus EB, Risch N, Thompson WD (1991): Genetic analysis of breast cancer in the cancer and steroid hormone study. Am J Hum Genet $\underline{48}, 232-242$

D'Amore PA, Thompson RW (1987): Mechanisms of angiogenesis. Annu Rev Physiol $\underline{49}, 453-464$

Davis PL, McCarty KS, Jr. (1997): Sensitivity of enhanced MRI for the detection of breast cancer: new, multicentric, residual, and recurrent. Eur Radiol 7 Suppl 5, 289-298

Delorme S (2001): Mammasonographie und Magnetresonanz-Mammographie als ergänzende Methoden im Mammographiescreening. Radiologe 41, 371-378

Duda V, Degenhardt F, Hahn D, Warm M: Mammasonographie; in: Stufe-3-Leitlinie Brustkrebs-Früherkennung in Deutschland; hrsg. v. Albert US; Zuckschwerdt, München 2008, 127-129

Eby PR, DeMartini WB, Gutierrez RL, Saini MH, Peacock S, Lehman CD (2009): Characteristics of probably benign breast MRI lesions. AJR Am J Roentgenol $\underline{193}, 861-867$

Fidler IJ, Ellis LM (1994): The implications of angiogenesis for the biology and therapy of cancer metastasis. Cell $\underline{79}, 185-188$

Fischer DR, Wurdinger S, Boettcher J, Malich A, Kaiser WA (2005): Further signs in the evaluation of magnetic resonance mammography: a retrospective study. Invest Radiol 40, 430-435

Fischer U: Lehratlas der MR - Mammographie, 1. Auflage; Thieme, Stuttgart 2000 
Fischer U (2008): Röntgenmammographie. Radiologe $\underline{48}$, 785-798

Fischer U: Lehratlas der MR - Mammographie, 2. Auflage; Thime, Stuttgart 2010

Fischer U, Helbich T: ACR BIRADS. Illustrierte Anleitung zur einheitlichen Befunderstellung von Mammographie, Mammasonographie und MR Mammographie, 1. Auflage; Thieme, Stuttgart 2006

Fischer U, v Heyden D, Vosshenrich R, Vieweg I, Grabbe E (1993): Signalverhalten maligner und benigner Läsionen in der dynamischen 2D-MRT der Mamma. Rofo $\underline{158}, 287-292$

Fischer U, Vosshenrich R, Probst A, Burchhardt H, Grabbe H (1994): Präoperative MRMammographie bei bekanntem Mammakarzinom. Sinnvolle Mehrinformation oder sinnloser Mehraufwand? Rofo $\underline{161}, 300-306$

Fischer U, Kopka L, Brinck U, Korabiowska M, Schauer A, Grabbe E (1997): Prognostic value of contrast-enhanced MR mammography in patients with breast cancer. Eur Radiol ㄱ, 1002-1005

Fischer U, Schwethelm L, Baum FT, Luftner-Nagel S, Teubner J (2009): Aufwand, Zuverlässigkeit und histologische Ergebnisse der MR-gesteuerten Vakuumbiopsie suspekter Mammabefunde - retrospektive Auswertung von 389 Interventionen. Rofo $\underline{181}$, 774-781

Folkman J (1971): Tumor angiogenesis: therapeutic implications. N Engl J Med $\underline{285}$, $1182-1186$

Friedrich M: Technik und Ergebnisse der Mammographie, 1. Auflage; Springer, Heidelberg 1993

Frykberg ER, Bland KI (1994): Overview of the biology and management of ductal carcinoma in situ of the breast. Cancer $\underline{74}, 350-361$

Giersiepen K, Heitmann C, Janhsen K, C L: Gesundheitsberichterstattung des Bundes: Brustkrebs, 1. Auflage; Robert-Koch-Institut, Berlin 2005

Gilles R, Guinebretiere JM, Lucidarme O, Cluzel P, Janaud G, Finet JF, Tardivon A, Masselot J, Vanel D (1994): Nonpalpable breast tumors: diagnosis with contrastenhanced subtraction dynamic MR imaging. Radiology 191, 625-631

Gordon PB (2002): Ultrasound for breast cancer screening and staging. Radiol Clin North Am $\underline{40}$, 431-441

Gualdi GF, Casciani E, Giuntini T, D'Amico D, Polettini E (2000): Imaging of the breast tissue. Clin Ter 151, 269-278 
Harms SE, Flamig DP, Hesley KL, Meiches MD, Jensen RA, Evans WP, Savino DA, Wells RV (1993): MR imaging of the breast with rotating delivery of excitation off resonance: clinical experience with pathologic correlation. Radiology 187, 493501

Heywang SH, Fenzl G, Beck R, Hahn D, Eiermann W, Permanetter W, Lissner J (1986): Anwendung von Gd-DTPA bei der kernspintomographischen Untersuchung der Mamma. Rofo $\underline{145}, 565-571$

Heywang SH, Wolf A, Pruss E, Hilbertz T, Eiermann W, Permanetter W (1989): MR imaging of the breast with Gd-DTPA: use and limitations. Radiology 171, 95-103

Heywang-Kobrunner SH (1994): Contrast-enhanced magnetic resonance imaging of the breast. Invest Radiol 29, 94-104

Heywang-Köbrunner S, Schreer I, Bässler R: Bildgebende Mammadiagnostik: Untersuchungstechnik, Befundungsmuster und Differentialdiagnostik in Mammographie, Sonographie und Kernspintomographie, 2. Auflage; Thieme, Stuttgart 2008

Holmich LR, Vejborg I, Conrad C, Sletting S, McLaughlin JK (2005): The diagnosis of breast implant rupture: MRI findings compared with findings at explantation. Eur $\mathrm{J}$ Radiol $\underline{53}, 213-225$

Horn F: Biochemie des Menschen, 2. Auflage; Thieme, Stuttgart 2003

Husmann G, Kaatsch P, Katalinic A, Bertz J, Haberland J, Kraywinkel K, Wolf U: Krebs in Deutschland 2005/2006 Häufigkeit und Trends, 7. Auflage; Robert KochInstitut, Saarbrücken 2010

Ikeda DM, Hylton NM, Kinkel K, Hochman MG, Kuhl CK, Kaiser WA, Weinreb JC, Viehweg P, Barclay J, Schnall MD (2001): Development, standardization, and testing of a lexicon for reporting contrast-enhanced breast magnetic resonance imaging studies. J Magn Reson Imaging $\underline{13}$, 889-895

Jayaram G, Swain M, Chew MT, Yip CH (2000): Cytologic appearances in invasive lobular carcinoma of the breast. A study of 21 cases. Acta Cytol $\underline{44}, 169-174$

Kaiser W: Signs in MR-Mammography, 1. Auflage; Springer, Heidelberg 2007

Kaiser W, Zeitler E (1986): Die Kernspintomographie der Mamma - Diagnose, Differentialdiagnose, Probleme und Lösungsmöglichkeiten. Rofo 144, 459-465

Kerlikowske K, Grady D, Barclay J, Sickles EA, Ernster V (1996): Effect of age, breast density, and family history on the sensitivity of first screening mammography. JAMA 276, 33-38

Kettritz U: Komplexe Mammadiagnostik, 1. Auflage; Uni.- Med Verlag AG, Bremen 2007 
Kettritz U, Rotter K, Schreer I, Murauer M, Schulz-Wendtland R, Peter D, HeywangKobrunner SH (2004): Stereotactic vacuum-assisted breast biopsy in 2874 patients: a multicenter study. Cancer $\underline{100}, 245-251$

Kolb TM, Lichy J, Newhouse JH (1998): Occult cancer in women with dense breasts: detection with screening US--diagnostic yield and tumor characteristics. Radiology 207, 191-199

Kolb TM, Lichy J, Newhouse JH (2002): Comparison of the performance of screening mammography, physical examination, and breast US and evaluation of factors that influence them: an analysis of 27,825 patient evaluations. Radiology $\underline{225}$, 165-175

Komatsu S, Lee CJ, Hosokawa Y, Ichikawa D, Hamashima T, Shirono K, Okabe H, Kurioka H, Oka T (2004): Comparison of intraductal spread on dynamic contrastenhanced MRI with clinicopathologic features in breast cancer. Jpn J Clin Oncol $\underline{34}, 515-518$

Kriege M, Brekelmans CT, Boetes C, Besnard PE, Zonderland HM, Obdeijn IM, Manoliu RA, Kok T, Peterse H, Tilanus-Linthorst MM (2004): Efficacy of MRI and mammography for breast-cancer screening in women with a familial or genetic predisposition. N Engl J Med 351, 427-437

Kuhl CK (2007): The current status of breast MR imaging. Part I. Choice of technique, image interpretation, diagnostic accuracy, and transfer to clinical practice. Radiology 244, 356-378

Kuhl CK, Bieling HB, Gieseke J, Kreft BP, Sommer T, Lutterbey G, Schild HH (1997): Healthy premenopausal breast parenchyma in dynamic contrast-enhanced MR imaging of the breast: normal contrast medium enhancement and cyclical-phase dependency. Radiology 203, 137-144

Kuhl CK, Klaschik S, Mielcarek P, Gieseke J, Wardelmann E, Schild HH (1999 a): Do T2-weighted pulse sequences help with the differential diagnosis of enhancing lesions in dynamic breast MRI? J Magn Reson Imaging $\underline{9}$, 187-196

Kuhl CK, Mielcareck P, Klaschik S, Leutner C, Wardelmann E, Gieseke J, Schild HH (1999 b): Dynamic breast MR imaging: are signal intensity time course data useful for differential diagnosis of enhancing lesions? Radiology 211, 101-110

Kuhl CK, Schmutzler RK, Leutner CC, Kempe A, Wardelmann E, Hocke A, Maringa M, Pfeifer U, Krebs D, Schild HH (2000): Breast MR imaging screening in 192 women proved or suspected to be carriers of a breast cancer susceptibility gene: preliminary results. Radiology $\underline{215}, 267-279$ 
Kuhl CK, Schild HH, Morakkabati N (2005): Dynamic bilateral contrast-enhanced MR imaging of the breast: trade-off between spatial and temporal resolution. Radiology 236, 789-800

Kuhl CK, Schrading S, Bieling HB, Wardelmann E, Leutner CC, Koenig R, Kuhn W, Schild HH (2007): MRI for diagnosis of pure ductal carcinoma in situ: a prospective observational study. Lancet $\underline{370}, 485-492$

Kuhl CK, Weigel S, Schrading S, Arand B, Bieling H, König R, Tombach B, Leutner C, Rieber-Brambs A, Nordhoff D (2010): Prospective Multicenter Cohort Study to Refine Management Recommendations for Women at Elevated Familial Risk of Breast Cancer: The EVA Trial. J Clin Oncol 로, 1450-1457

Kurz K, Hanstein B, Mödder U, Bender H (2006): MRT in der Mammadiagnostik. Gynakologe $\underline{39}, 524-532$

Lanyi M (1982): Formanalye von 153 Mikroverkalkungsgruppen maligner Genese: "Das Dreickprinzip". Rofo 136, 77-84

Lebeau A, Kreipe H, Schlake W: Anleitung Mammapathologie; in: Stufe-3-Leitlinie Brustkrebs-Früherkennung in Deutschland; hrsg. v. Albert US; Zuckschwerdt, München 2008, 251-297

Leonhardt H: Histologie, Zytologie und Mikroanatomie des Menschen, 7. Auflage; Thieme, Stuttgart 1985

Lewin B: Molekularbiologie der Gene, 6. Auflage; Spektrum Akademischer Verlag, Heidelberg 1998

Liberman L, Abramson AF, Squires FB, Glassman JR, Morris EA, Dershaw DD (1998): The breast imaging reporting and data system: positive predictive value of mammographic features and final assessment categories. AJR Am J Roentgenol $\underline{171}, 35-40$

Liberman L, Morris EA, Benton CL, Abramson AF, Dershaw DD (2003 a): Probably benign lesions at breast magnetic resonance imaging: preliminary experience in high-risk women. Cancer $\underline{98}, 377-388$

Liberman L, Morris EA, Dershaw DD, Abramson AF, Tan LK (2003 b): MR imaging of the ipsilateral breast in women with percutaneously proven breast cancer. AJR Am J Roentgenol 180, 901-910

Liberman L, Mason G, Morris EA, Dershaw DD (2006): Does size matter? Positive predictive value of MRI-detected breast lesions as a function of lesion size. AJR Am J Roentgenol 186, 426-430 
Macura KJ, Ouwerkerk R, Jacobs MA, Bluemke DA (2006): Patterns of enhancement on breast MR images: interpretation and imaging pitfalls. Radiographics $\underline{26}, 1719-$ 1734

Madjar H, Ohlinger R, Mundinger A, Watermann D, Frenz JP, Bader W, SchulzWendtland R, Degenhardt F (2006): BI-RADS-analogue DEGUM criteria for findings in breast ultrasound--consensus of the DEGUM Committee on Breast Ultrasound. Ultraschall Med $\underline{27}$, 374-379

Malich A, Fischer DR, Wurdinger S, Boettcher J, Marx C, Facius M, Kaiser WA (2005): Potential MRI interpretation model: differentiation of benign from malignant breast masses. AJR Am J Roentgenol $\underline{185}$, 964-970

Mandelson MT, Oestreicher N, Porter PL, White D, Finder CA, Taplin SH, White E (2000): Breast density as a predictor of mammographic detection: comparison of interval- and screen-detected cancers. J Natl Cancer Inst $\underline{\text { 92, }}$ 1081-1087

Müller-Schimpfle M (2008): Consensus meeting of course experts in breast diagnosis 5 May 2007 in Frankfurt am Main--topic: microcalcinosis. Rofo 180, 66-68

Nunes LW, Schnall MD, Orel SG, Hochman MG, Langlotz CP, Reynolds CA, Torosian MH (1997): Breast MR imaging: interpretation model. Radiology 202, 833-841

Olivotto IA, Kan L, d'Yachkova Y, Burhenne LJ, Hayes M, Hislop TG, Worth AJ, Basco VE, King S (2000): Ten years of breast screening in the Screening Mammography Program of British Columbia, 1988-97. J Med Screen $\underline{7}$, 152-159

Order BM, Schreer I (2008): Früherkennung des Mammakarzinoms. Der Onkologe $\underline{14}$, 120-130

Orel SG, Schnall MD, LiVolsi VA, Troupin RH (1994): Suspicious breast lesions: MR imaging with radiologic-pathologic correlation. Radiology 190, 485-493

Orel SG, Kay N, Reynolds C, Sullivan DC (1999): BI-RADS categorization as a predictor of malignancy. Radiology 211, 845-850

Orel SG, Rosen M, Mies C, Schnall MD (2006): MR imaging-guided 9-gauge vacuumassisted core-needle breast biopsy: initial experience. Radiology 238, 54-61

Ortmann O, Albert U, Schulz K: Risikofaktoren; in: Stufe-3-Leitlinie BrustkrebsFrüherkennung in Deutschland; hrsg. v. Albert U; Zuckschwerdt, München 2008, 50-54

Perlet C, Heywang-Kobrunner SH, Heinig A, Sittek H, Casselman J, Anderson I, Taourel $P$ (2006): Magnetic resonance-guided, vacuum-assisted breast biopsy: results from a European multicenter study of 538 lesions. Cancer 106, 982-990 
Pisano ED, Gatsonis C, Hendrick E, Yaffe M, Baum JK, Acharyya S, Conant EF, Fajardo LL, Bassett L, D'Orsi C (2005): Diagnostic performance of digital versus film mammography for breast-cancer screening. N Engl J Med $\underline{353}, 1773-1783$

Rosenberg RD, Hunt WC, Williamson MR, Gilliland FD, Wiest PW, Kelsey CA, Key CR, Linver MN (1998): Effects of age, breast density, ethnicity, and estrogen replacement therapy on screening mammographic sensitivity and cancer stage at diagnosis: review of 183,134 screening mammograms in Albuquerque, New Mexico. Radiology 209, 511-518

Sadowski EA, Kelcz F (2005): Frequency of malignancy in lesions classified as probably benign after dynamic contrast-enhanced breast MRI examination. J Magn Reson Imaging 21, 556-564

Sardanelli F, Giuseppetti GM, Panizza P, Bazzocchi M, Fausto A, Simonetti G, Lattanzio V, Del Maschio A (2004): Sensitivity of MRI versus mammography for detecting foci of multifocal, multicentric breast cancer in Fatty and dense breasts using the whole-breast pathologic examination as a gold standard. AJR Am J Roentgenol $\underline{183}, 1149-1157$

Schleicher UM, Ammon J (1998): Mode of breast cancer detection: a study from the German part of the Maas-Rhine-EUREGIO. Eur J Cancer Prev $\underline{7}, 41-46$

Schnall MD (2001): Application of magnetic resonance imaging to early detection of breast cancer. Breast Cancer Res $\underline{3}, 17-21$

Schnall MD, Rosten S, Englander S, Orel SG, Nunes LW (2001): A combined architectural and kinetic interpretation model for breast MR images. Acad Radiol $\underline{8}, 591-597$

Schnall MD, Blume J, Bluemke DA, DeAngelis GA, DeBruhl N, Harms S, HeywangKobrunner SH, Hylton N, Kuhl CK, Pisano ED (2006): Diagnostic architectural and dynamic features at breast MR imaging: multicenter study. Radiology $\underline{238}$, $42-53$

Schorn C, Fischer U, Luftner-Nagel S, Grabbe E (1999): Diagnostic potential of ultrafast contrast-enhanced MRI of the breast in hypervascularized lesions: are there advantages in comparison with standard dynamic MRI? J Comput Assist Tomogr $\underline{23}, 118-122$

Schreer I, Altland H, Engel J, Hölzel D, Mülelr-Schimpfle M: Mammographie als Teil der Diagnosekette; in: Stufe-3-Leitlinie Brustkrebs-Früherkennung in Deutschland; hrsg. v. Albert U; Zuckschwerdt, München 2008, 119-126

Schulz-Wendtland R, Becker N, Bock K, Anders K, Bautz W (2007): Mammographiescreening. Radiologe $\underline{47}, 359-370$ 
Sickles EA (1991): Periodic mammographic follow-up of probably benign lesions: results in 3,184 consecutive cases. Radiology $\underline{179}$, 463-468

Sickles EA (1995): Management of probably benign breast lesions. Radiol Clin North Am $\underline{33}, 1123-1130$

Siegmann KC, Muller-Schimpfle M, Schick F, Remy CT, Fersis N, Ruck P, Gorriz C, Claussen CD (2002): MR imaging-detected breast lesions: histopathologic correlation of lesion characteristics and signal intensity data. AJR Am J Roentgenol 178, 1403-1409

Siegmann KC, Moron HU, Baur A, Hahn M, Vogel U, Claussen CD, Bitzer M (2009): Diagnostische Wertigkeit des Göttinger Scores zur Malignitätsvorhersage von ausschließlich in der MRT darstellbaren Mammaläsionen. Rofo 181, 556-563

Silverstein MJ, Poller DN, Waisman JR, Colburn WJ, Barth A, Gierson ED, Lewinsky B, Gamagami P, Slamon DJ (1995): Prognostic classification of breast ductal carcinoma-in-situ. Lancet $\underline{345}, 1154-1157$

Smith RA, Saslow D, Sawyer KA, Burke W, Costanza ME, Evans WP, 3rd, Foster RS, Jr., Hendrick E, Eyre HJ, Sener S (2003): American Cancer Society guidelines for breast cancer screening: update 2003. CA Cancer J Clin $\underline{53}, 141-169$

Tabar L, Fagerberg G, Duffy SW, Day NE, Gad A, Grontoft O (1992): Update of the Swedish two-county program of mammographic screening for breast cancer. Radiol Clin North Am $\underline{30}, 187-210$

Tabar L, Vitak B, Chen HH, Duffy SW, Yen MF, Chiang CF, Krusemo UB, Tot T, Smith RA (2000): The Swedish Two-County Trial twenty years later. Updated mortality results and new insights from long-term follow-up. Radiol Clin North Am $\underline{38}, 625$ 651

Tavassoli F, Hoefler H, Rosai J, Holland R, Ellis I, Schnitt S, Boecker W, HeywangKöbrunner S, Moinfar F, Lakhani S: Intraductal proliferative lesions; in: Pathology and Genetics Tumours of the Breast and Female Genital Organs; hrsg. v. Tavassoli F und Devilee P; IARC Press, Lyon 2003, 9-110

Tulusan AH, Bühner M, Parchent H, Rinas N, Kastner P (2005): Duktales Carcinoma in situ. Gynakologe $\underline{38}, 65-74$

Urdl W, Auner H, Giuliani A (1996): Der Sinn einer Hormonsubstitution im Klimakterium der Frau. Eur Surg 28, 256-259

Varas X, Leborgne F, Leborgne JH (1992): Nonpalpable, probably benign lesions: role of follow-up mammography. Radiology $\underline{184}$, 409-414 
Varas X, Leborgne JH, Leborgne F, Mezzera J, Jaumandreu S (2002): Revisiting the mammographic follow-up of BI-RADS category 3 lesions. AJR Am J Roentgenol $\underline{179}, 691-695$

Vizcaino I, Gadea L, Andreo L, Salas D, Ruiz-Perales F, Cuevas D, Herranz C, Bueno F (2001): Short-term follow-up results in 795 nonpalpable probably benign lesions detected at screening mammography. Radiology $\underline{219}$, 475-483

Warren RM, Thompson D, Pointon LJ, Hoff R, Gilbert FJ, Padhani AR, Easton DF, Lakhani SR, Leach MO (2006): Evaluation of a prospective scoring system designed for a multicenter breast MR imaging screening study. Radiology $\underline{239}$, 677-685

Wedegärtner U, Bick U, Wortler K, Rummeny E, Bongartz G (2001): Differentiation between benign and malignant findings on MR-mammography: usefulness of morphological criteria. Eur Radiol 11, 1645-1650

Wittekind C: Brustdrüse; in: Allgemeine und spezielle Pathologie; hrsg. v. Riede U und Schaefer H; Thieme, Stuttgart 2004, 967-980

Yancopoulos GD, Davis S, Gale NW, Rudge JS, Wiegand SJ, Holash J (2000): Vascular-specific growth factors and blood vessel formation. Nature $\underline{407}, 242-248$ 


\section{Danksagungen}

Mein besonderer Dank geht an Herrn Prof. Dr. med. Uwe Fischer, für seine stete Diskussionsbereitschaft und Unterstützung bei der Durchführung dieser Arbeit. Auch danke ich ihm, dass er mir die Freude und das Interesse am wissenschaftlichen Arbeiten nahe gebracht hat. 


\section{Curriculum vitae}

Als Sohn von Anke Ruhland, Zahnärztin, und Peter Ruhland, Zahnarzt, wurde ich am 26. September 1984 in Göttingen geboren.

Als Schüler des Max-Planck-Gymnasiums in Göttingen erhielt ich im Jahre 2004 die allgemeine Hochschulreife. Den Zeitraum von Sommer 2001 bis Sommer 2002 verbrachte ich als Austauschschüler an der West Orange School in Orlando, Florida. Dort erwarb ich meinen amerikanischen High-School-Abschluss.

Nach dem Abitur absolvierte ich den Wehrersatzdienst im evangelischen Krankenhaus Neu-Bethlehem in Göttingen.

Im Wintersemester 2005 begann ich das Studium der Zahnheilkunde an der Universität Leipzig. Nach Abschluss der naturwissenschaftlichen Vorprüfung wechselte ich zum Wintersemester 2006 an die Albertus Magnus Universität zu Köln. Im Frühjahr 2006 absolvierte ich dort die zahnmedizinische Vorprüfung und im Winter 2010 die zahnärztliche Prüfung. Im Dezember 2010 erhielt ich meine Approbation als Zahnarzt.

In dem Zeitraum vom Sommer 2009 bis Sommer 2011 arbeitete ich als Doktorand im diagnostischen Brustzentrum Göttingen unter der Leitung von Herrn Prof. Dr. med. Uwe Fischer.

Im Mai 2011 trat ich eine Stelle als zahnärztlicher Vorbereitungsassistent in der Zahnarztpraxis von Dr. Mathias Eubisch in Erftstadt an. 